\title{
Abstracts of the Ninth Annual Meeting of the Lumbar Spine Research Society \\ Chicago, Illinois • April 14-15, 2016 \\ (DOI: 10.3171/2016.4.FOC-LSRSabstracts)
}

\begin{abstract}
Paper \#1. How Effective is Physical Therapy for Low Back Pain? A Multivariate Analysis of 4597 Patients.
\end{abstract}

Ananth Eleswarapu, M.D. ${ }^{1}$, Srikanth Divi, M.D. ${ }^{2}$, Douglas Dirschl, M.D. ${ }^{1}$, James Mok, M.D. ${ }^{1}$, Christopher Stout, Ph.D. ${ }^{3}$, Michael Lee, M.D. ${ }^{l}$

${ }^{1}$ University of Chicago Medical Center, Chicago, IL ${ }^{2}$, Chicago, IL, ${ }^{3}$ ATI Physical Therapy, Bolingbrook, IL

Background/Introduction: Axial low back pain is a major cause of morbidity and disability in health care. Previous studies have found questionable efficacy for surgery in the absence of specific indications (instability, neoplasm, infection, etc.). Nonoperative treatments are available, however there is scant evidence as to the efficacy of PT and risk factors on outcomes. The purpose of this study is to evaluate whether the treatment of nonspecific low back pain with physical therapy (PT) results in clinically significant improvements in patient-reported pain and functional outcomes.

Materials/Methods: 4597 patients who underwent PT for low back pain were identified from the registry of a commercial physical therapy organization. The primary outcome measures were pre- and post-treatment scores on the Oswestry Disability Index (ODI), activity Numeric Pain Rating Scale (NPRS), and resting NPRS. Previously published thresholds for minimum clinical important difference (MCID) were used to determine the proportion of patients meeting MCID for each of our outcomes. Logistic regression analysis was used to determine risk factors predictive of treatment failure.

Results: The average number of visits was 14 and average duration of therapy was 45 days. Treated diagnoses included lumbar sprain, lumbar disc displacement, lumbosacral spondylosis, and lumbago. $28.5 \%$ of patients met the MCID for improvement in ODI. Night symptoms, obesity, and smoking were predictors of treatment failure for ODI. 59\% of patients met the MCID for improvement in resting NPRS, with history of venous thromboembolism, night symptoms, psychiatric disease, workers' compensation status, smoking, and obesity predictive of treatment failure. $60 \%$ of patients met the MCID for improvement in activity NPRS, with night symptoms, workers' compensation status, and smoking predictive of treatment failure.

Discussion/Conclusion: The treatment of low back pain continues to be challenging. $40-72.5 \%$ of the population did not meet MCID for pain and function following treatment of low back pain with PT. Common risk factors for treatment failure included smoking, workers' compensation, night pain, and psychiatric history. Though PT offers low risk when compared to more invasive treatments of low back pain, further study on the clinical and cost effectiveness of PT for common low back pain diagnoses is needed.
Paper \#2. Is the readability of spine-related patient education material improving? An assessment of subspecialty websites

Adam Eltorai, $B A^{1}$, Mark Palumbo, $M D^{2}$, Alan Daniels, $M D^{3}$

${ }^{1}$ Warren Alpert Medical School of Brown University, Providence, RI, ${ }^{2}$ Department of Orthopaedics, Division of Spine Surgery, Warren Alpert Medical School of Brown University, Providence, RI, ${ }^{3}$ Department of Orthopaedics, Division of Spine Surgery-Adult Spinal Deformity Service, Warren Alpert Medical School of Brown University, Providence, Rhode Island

Background/Introduction: Many spine patients utilize the Internet to acquire health information. Several agencies recommend that the readability of online patient education materials should be no greater than a sixth-grade reading level (1112 years), as health literacy predicts health-related quality of life outcomes. The publicly available patient education information on the North American Spine Society (NASS), American Association of Neurological Surgeons (AANS), and American Academy of Orthopaedic Surgeons (AAOS) websites may be too complex for some patients to understand. This study evaluated whether the subspecialty online patient education materials meet recommended readability guidelines for medical information.

Materials/Methods: All publicly accessible spine-related entries within the patient education section of the NASS, AANS, and AAOS websites were analyzed for grade level readability using the Flesch-Kincaid formula, a widely-used and validated tool to evaluate the text reading level. Readability scores were also compared to a similar 2008 analysis. Comparative statistics were performed.

Results: A total of 125 entries from the three subspecialty websites were analyzed. The average (SD) readability of the online articles was grade level 10.7 (2.3). One hundred seventeen of the articles $(93.6 \%)$ had a readability score above the sixth-grade level, the maximum level recommended by several healthcare agencies. The readability of the articles exceeded the maximum recommended level by an average of 4.7 grade levels $(95 \% \mathrm{CI}$, 4.292 to $5.103 ; \mathrm{P}<0.0001)$. Compared to 2008 , the three societies published more spine-related patient education articles (61 vs. 125, $\mathrm{P}=0.0454)$ and the average readability level improved from 11.5 to $10.7(\mathrm{P}=0.0184)$.

Discussion/Conclusion: Our findings suggest that the spine-related patient education materials on the NASS, AAOS, and AANS websites have readability levels that may make comprehension difficult for a substantial portion of the patient population. While some progress has been made in the readability of patient education materials over the past seven years, additional improvement is necessary. 
Paper \#3. Cost-Effectiveness of Lumbar Epidural Steroid Injections

Marco Swanson, B.S. ${ }^{1}$, Vikram Mehta, M.P.H. ${ }^{1}$, Matthew Alvin, M.D. ${ }^{2}$; Heather Fuhrman, B.S. ${ }^{1}$, Daniel Lubelski, M.D. ${ }^{2}$, Edward Benzel, M.D. ${ }^{2}$, Thomas Mroz, M.D. ${ }^{2}$

${ }^{1}$ Case Western Reserve University School of Medicine, Cleveland, $\mathrm{OH},{ }^{2}$ Cleveland Clinic Foundation, Neurological Institute, Cleveland, $\mathrm{OH}$

Background/Introduction: Epidural steroid injections (ESIs) are a commonly used treatment strategy for low back pain and lumbar radiculopathy. However, their cost-effectiveness and the impact that they have on quality of life (QOL) outcomes is debated. The purpose of the present study was to analyze the cost-effectiveness of lumbar epidural steroid injections (ESIs) compared to conservative management alone for patients with lumbar radiculopathy and low back pain.

Materials/Methods: QOL measures included the EuroQol-5 (EQ-5D) questionnaire, Pain Disability Questionnaire (PDQ), Patient Health QUestionnaire-9 (PHQ-9), and the Visual Analogue Scale (VAS). QOL outcomes were collected pre-intervention and after 3 and 6 months. Cost estimations were based on Medicare national payment amounts, patient income and missed workdays. The cost-utility and incremental cost effectiveness ratios (ICER) were calculated at the 3-month and 6-month follow-up and assessed for cost-effectiveness using the $\$ 100,000$ / Quality-adjusted life year (QALY) gained threshold converted to these time intervals.

Results: At 3 months, there was a significant improvement in EQ-5D scores in the ESI cohort $(\triangle \mathrm{EQ}-5 \mathrm{D}=0.06 ; \mathrm{p}=0.03)$ as well as in the conservative cohort $(\triangle \mathrm{EQ}-5 \mathrm{D}=0.07 ; \mathrm{p}=0.03)$, but no significant difference in improvement between cohorts. At 3 months, there was no significant difference $(p=0.18)$ between the total costs (direct and indirect costs) incurred by the ESI cohort $(\$ 2,190)$ versus the conservative cohort $(\$ 1,772)$. At 3 months, the cost utility ratios for the ESI cohort and conservative cohort were $\$ 38,710$ and $\$ 27,313$ per QALY gained, respectively $(\mathrm{p}=0.73)$. The ICERs revealed that ESIs are economically dominated at 3-months due to the smaller, but statistically insignificant increase in QALY gained in the ESI cohort versus conservative cohort. At 6 months, there was no statistically significant change in EQ-5D scores from baseline in either cohort or between cohorts.

Discussion/Conclusion: ESIs are not cost-effective at either the 3-month or 6-month follow-up period. At 3 months, ESIs provide an equal improvement in QOL outcomes compared to conservative management and at similar costs. At 6 months, neither ESIs nor conservative management provide an improvement in QOL outcomes.

Paper \#4. Is There An Association of Epidural Corticosteroid Injection with Postoperative Surgical Site Infection after Surgery for Lumbar Degenerative Spine Disease?

Jenis Louis, $M D^{l}$, Stefan Hartveldt, BSc, BHS', Stein Janssen, $M D^{3}$, Kirkham Wood, $\mathrm{MD}^{4}$, Thomas Cha, $\mathrm{MD}, \mathrm{MBA}^{3}$, Joseph Schwab, $M D, M S^{3}$

${ }^{1}$ Massachusetts General Hospital, Boston, MA, ${ }^{2}$, Boston, MA, ${ }^{3} \mathrm{MGH}$, Boston, MA, ${ }^{4}$, Redwood City, CA

Background/Introduction: The effect of local steroid injections on the occurrence of surgical site infections is controversial. This retrospective study aimed to assess the temporal relationship between exposure to epidural steroid injection before surgery and postoperative surgical site infection (SSI) within three months after surgery for degenerative lumbar spine conditions.

Materials/Methods: Patients who underwent decompressive laminectomy and/or arthrodesis for degenerative lumbar spine conditions at two affiliated tertiary care centers between 2005 and 2015 were identified. Inclusion criteria were patients 18 years or older with at least 90 days of clinical follow-up. Exclusion criteria: tumor, fracture, trauma, pseudarthrosis, or preexisting infection. Primary outcome measure was SSI within 90 days after surgery requiring incision and debridement.

Results: 5311 patients (age $57+/-16$ years) were analyzed of which $945(18 \%)$ had at least one epidural steroid injection within 90 days of surgery (average 1.33 injections $+/-0.56$ ). One hundred thirty-four $(2.5 \%)$ patients developed a surgical site infection requiring reoperation. No association of exposure or dose-response relationship was identified between ESI and SSI for any of the time periods (90-day, 30-day, and 30 to 90-day). Five $(1.7 \%)$ of the 290 patients who had at least one ESI within 30 days before surgery had a postoperative infection compared to $129(2.6 \%)$ of the 5021 in the no-ESI group (unadjusted odds ratio: $0.67,95 \% \mathrm{CI}: 0.27-1.64, \mathrm{p}=0.376)$. Fifteen $(2.0 \%)$ of the 761 patients who had at least one ESI within 30 to 90 -days before surgery had a postoperative infection, compared to $119(2.6 \%)$ of the 4550 in the no-ESI group (unadjusted odds ratio: $0.75,95 \% \mathrm{CI}$ : $0.44-1.29, \mathrm{p}=0.296$ ).

Discussion/Conclusion: In this retrospective cohort study we investigated whether an epidural steroid injection and its timing influences the postoperative risk of a surgical site infection. We found no association-exposure or dose-response relationshipbetween ESI and postoperative infection, even after adjusting for potential confounders, for any of the time periods (90-day, 30-day, and 30 to 90-day ESI). Additionally, we did find that longer hospital stay, greater EBL, posterior approach, and drain placement were associated with higher infection rates.

Paper \#5. Validation of Patient Reported Outcomes Measurement Information System (PROMIS) in Lumbar Stenosis

Alpesh Patel, MD, FACS ${ }^{1}$, Shah-Nawaz Dodwad, $M D^{2}$; Surabhi Bhatt, BS ${ }^{1}$, Jason Savage, M.D. ${ }^{3}$, Wellington Hsu, M.D. ${ }^{4}$, Nan Rothrock, $P h D^{2}$

${ }^{1}$ Northwestern University, Chicago, IL, ${ }^{2}$, Chicago, IL, ${ }^{3}$ The Cleveland Clinic, Cleveland, Ohio, ${ }^{4}$ Northwestern Memorial Hospital, Chicago, Illinois

Background/Introduction: PROMIS $®$ is a National Institutes of Health (NIH) funded adaptive, responsive assessment tool that measures patient-reported health status. The objective of this project was to validate PROMIS ${ }^{\circledR}$ measures for pain behavior, pain interference, and physical function in surgically treated patients with lumbar spinal stenosis against historical outcomes including Zurich Claudication Questionnaire (ZCQ), Oswestry Disability Index (ODI), and the Short-Form 12 (SF-12).

Materials/Methods: Computer adaptive testing of PROMIS $₫$ (pain behavior, pain interference, and physical function domains), ODI, ZCQ, and SF-12 outcome measures were administered to 92 consecutive tertiary hospital patients treated surgically for lumbar spinal stenosis. Assessments were done at baseline (preoperatively) and postoperatively at 6 weeks and 3 months. We excluded patients presenting for a revision surgery, tumor, infection, or trauma. Each patient prospectively completed the PROMIS ${ }^{\circledR}$ assessments and legacy measures custom built into an online Assessment Center by using a secure login and password on a Wi-Fi enabled tablet.

Results: Of the 92 patients enrolled (mean age of 62.2, 58 male, 
34 female), PROMIS $\left({ }^{\circ}\right.$ measures showed statistically significant improvement in outcome scores similar to ODI, ZCQ, and SF-12 at all-time points with Pearson correlation coefficients ranging from absolute values of .27-.73 at baseline and .27-.70 between 6 weeks and 3 months. Compared to their prior visit at 6 weeks, at 3 months postoperatively, 27 patients of 35 patients $(77.1 \%)$ reported additional improvement in their preoperative condition. At baseline and throughout the assessment, both ZCQ (0-25\%) and SF-12 (0-45\%) had more patients in the floor and ceiling areas compared to PROMIS $®(0-1 \%)$ and ODI (0). The PROMIS $®$ CATs were completed on average in 2.6 minutes compared to 9.7 minutes for historical measures. Concurrent medical conditions that contributed to responses were present in $30 \%$ of patients.

Discussion/Conclusion: PROMIS® is a valid and responsive measure of surgically treated patients with lumbar spinal stenosis when compared to legacy ODI, SF-12 and Zurich patient outcomes. PROMIS $®$ may be preferable to legacy instruments because of the efficacy in measuring treatment effect, ability to accurately evaluate multiple parameters, and avoidance of floor/ ceiling effects.

Paper \#6. Spine SCOAP at 3 Years: Adding Value in Spine Surgery through a Surgeon-led QI Initiative

Paul Anderson, $M D^{I}$, Neal Shonnard, MD', Jens Chapman, $M D^{3}$, Amy Cizik, PhD ${ }^{3}$, Carlo Bellabarba, $M D^{3}$, Rod Oskouian, $M D^{3}$, Rajiv Sethi, $M D^{3}$, Christoph Hofsetter, $M D^{3}$, Samuel Bederman, $\mathrm{MD}^{4}$

${ }^{1}$ University of Wisconsin, Madison, WI, ${ }^{2}$, Tacoma, WA, ${ }^{3}$, Seattle, WA, ${ }^{4}$, Plano, TX

Background/Introduction: The Surgical Care and Outcomes Assessment Program (SCOAP) developed a spine surgery quality improvement module, Spine SCOAP, in 2010. The purpose of this study is to review challenges encountered in developing Spine SCOAP, the strategies deployed in sustaining the program and to report measurable improvements in quality of care in the first 3 years.

Materials/Methods: Spine SCOAP prospectively gathers observational data from 20 hospitals across WA State. Trained abstractors collect patient experience data by a multimodal survey center. SS captures longitudinal QI surveillance data patients undergoing spine fusion and a random sample non-fusion procedures. Linking SS data to hospital discharge data allows for tracking on all re-hospitalizations and charge information. Charges were adjusted for consumer price index to 2011 and converted from charges to costs using hospital specific conversion factors.

Results: 34,729 statewide procedures were recorded in WA State though state-run database. Spine SCOAP recorded over $27,674(79.7 \%)$ procedures from 20 hospitals including $100 \%$ of spine fusion procedures and approximately $30 \%$ of non-fusion procedures. The costs of index admission increased significantly between 2011 and 2013 at non-SS hospitals compared to cost changes at SS hospitals (1\% increase at non-SS hospitals vs $6 \%$ decrease at SS hospitals, $\mathrm{p}<0.01$ Figure 1). 90-day reoperation rates were significantly lower in SS hospitals compared to nonSS hospitals $(2.4 \%$ at non-SS hospitals vs $1.6 \%$ at SS hospitals, $\mathrm{p}<0.001$-Figure 2). Costs were largely driven by significant reductions in 90-day readmission and index cost reductions related to decreases in adverse outcome.

Discussion/Conclusion: Spine SCOAP's novel, surgeon-led approach to QI has accomplished remarkable success in a few short years. Reductions in rates of reoperation and costs of care support the role of clinicians in leading QI initiatives to add value to spine care. Challenges to the successful implementation of such programs include alignment of stakeholder interests, engagement of clinicians and payers. Spine SCOAP at 3 years highlights the success that follows collaborative relationships based on trust between payers, physicians and patients towards meaningful QI and may be a model for those interested in adding value to spine surgery.

Paper \#7. Do Lumbar Decompression and Fusion Patients Recall Their Preoperative Status? Recall Bias in PatientReported Outcomes

Ilyas Aleem, MD, MSc, FRCSC ${ }^{l}$, Jonathan Duncan, $M D^{l}$, Amin Mohamed Ahmed, $M D^{l}$, Jason Eck, $M D^{2}$, Rhee John, $M D^{3}$, Michelle Clarke, $\mathrm{MD}^{4}$, Bradford Currier, M.D. ${ }^{4}$, Ahmad Nassr, $M D^{l}$

${ }^{1}$ Mayo Clinic, Rochester, MN, ${ }^{2}$ Chattanooga Center for Sports Medicine and Orthopedics, Chattanooga, TN, ${ }^{3}$ Emory Health, Atlanta, GA, ${ }^{4}$, Rochester, MN

Background/Introduction: Accurate patient recollection of preoperative symptoms may limit the interpretability of patientreported outcomes (PROs). We sought to characterize the accuracy of patient recall of preoperative symptoms in a cohort of lumbar spine surgery patients.

Materials/Methods: We analyzed consecutive patients undergoing lumbar decompression or decompression and fusion for lumbar radiculopathy by a single surgeon over a four-year period. Using standardized questionnaires, we recorded back and leg numeric pain scores (NPS) and Oswestry Disability Indices (ODI) preoperatively and asked patients to recall their preoperative status at a minimum of one-year following surgery. We compared actual and recalled preoperative scores. Multivariable linear regression was used to identify factors that had an impact on recollection. Pearson correlation coefficients quantified relations between recalled and actual scores.

Results: Sixty-two patients with a mean age of 66.1 years (55\% female) were included. Compared to their preoperative scores, patients showed significant improvement in back pain (mean difference $(\mathrm{MD})=-3.2,95 \% \mathrm{CI}-4.0$ to $-2.4, \mathrm{p}<0.01$ ), leg pain (MD $-3.3,95 \% \mathrm{CI}-4.3$ to $-2.2, \mathrm{p}<0.01$ ), and disability (MD $-25.0 \%, 95 \%$ CI -28.7 to $-19.6, p<0.01)$. Patient recollection of preoperative status was significantly more severe than their actual preoperative status for back pain $(\mathrm{MD}+2.3,95 \%$ CI 1.5 to 3.2 , $\mathrm{p}<0.05)$, leg pain (MD $+1.8,95 \%$ CI 0.9 to 2.7 , p < 0.05$)$ and disability (MD $+9.6 \%, 95 \%$ CI 5.6 to $14.0, \mathrm{p}<0.05)$. This was maintained across age, gender, and duration of symptoms prior to surgery. The magnitude of recall bias was moderate to severe and exceeded the minimal clinically important difference (MCID) in more than $67 \%$ of patients for back and leg pain. No significant correlation between actual and recalled scores with regards to back $(\mathrm{r}=0.18)$ or leg pain $(\mathrm{r}=0.24)$ was seen.

Discussion/Conclusion: Patient recollection of preoperative status one year after lumbar spine surgery was significantly more severe than their actual preoperative status for back pain, leg pain, and disability. These findings indicate that relying on patient recollection does not provide an accurate measure of preoperative status, limiting the interpretation and applicability of PROs. 
Paper \#8. Patient Satisfaction Is Not Associated with Pain Medication Consumption in Postoperative Spine Surgery Patients in the Inpatient Setting

Miranda Bice, MD, Amir Abtahi, MD, Dane Barton, MS, Angela Presson, PhD, Qian Ding, PhD, Brandon Lawrence, MD, Darrel Brodke, MD, W. Ryan Spiker, MD

\section{, Salt Lake City, Utah}

Background/Introduction: Despite increased focus on patient satisfaction as a measure of healthcare quality, the factors that influence it are incompletely understood. The aims of this study were to determine the relationships between patient-reported pain, inpatient pain medication consumption, satisfaction with pain control, and overall satisfaction with admission postoperatively.

Materials/Methods: A retrospective review was performed for all patients admitted following spinal surgery with a completed Hospital Consumer Assessment of Healthcare Providers and Systems (HCAHPS) survey between December 2010 and March 2014. Daily averages of patient-reported pain using the numerical pain rating scale (NPRS), narcotic usage in morphine equivalents, alternative pain medication usage, and patient-reported satisfaction obtained from HCAHPS surveys were recorded. Statistical analyses were performed to evaluate for associations among these variables.

Results: Of the 316 patients meeting the inclusion criteria, $49.7 \%$ reported that pain was "always" controlled and $73.8 \%$ were "satisfied" with the hospital admission based on HCAHPS survey responses. There was no significant association observed between type or amount of pain medication use, and satisfaction with pain control nor the hospital ( $p>0.05)$ (Table 1). The average NPRS scores for patients that were versus those that were not satisfied was nearly identical (3.16 vs. 3.19, $\mathrm{p}=0.89$ ). The perception that staff did everything they could to provide pain control was significantly associated with hospital satisfaction $(\mathrm{p}<0.001)$.

Discussion/Conclusion: Patient-reported measures, including satisfaction, are multifactorial and affected by several factors including pain control, care team efforts, patient-specific characteristics, and psychosocial factors. Greater opioid consumption was associated with higher pain scores but not with satisfaction with pain control, suggesting that administration of increased amounts of opioids may not improve satisfaction. However, the significant association observed between care team efforts and satisfaction allude to the importance of providerpatient interactions between. This study demonstrates that increased narcotic consumption is not associated with greater pain satisfaction while perception by patients of efforts provided by the care team to manage pain is associated with satisfaction. These results emphasize the importance of open communication with patients regarding their experiences and treatment plans while questioning the assumption the provisions of greater amount of narcotics will increase satisfaction.

Paper \#9. Effects of Intraoperative Anesthetic Medications on Postoperative Urinary Retention after Single Level Lumbar Fusion

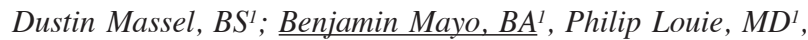
Daniel Bohl, MD, MPH ${ }^{2}$, Stephanie Iantorno, BS ${ }^{l}$, Junyoung Ahn, $B S^{l}$, Ehsan Tabaraee, $M D^{3}$, Kern Singh, $M D^{l}$

${ }^{1}$ Rush University Medical Center, Chicago, IL, ${ }^{2}$ Rush University Medical Center, New Haven, CT, ${ }^{3}$ Stanislaus Orthopaedics, Modesto, CA

Background/Introduction: Postoperative urinary retention
(POUR) is a concerning event that may occur following routine orthopaedic surgery. The purpose is to identify medications that may potentially contribute to an increased likelihood of developing POUR following lumbar spinal fusion procedures.

Materials/Methods: A prospectively collected registry of patients that underwent a primary single-level, minimally invasive transforaminal lumbar interbody fusion between 2009-2013 was reviewed. POUR was defined as a bladder scan of $\geq 300 \mathrm{ml}$, the post-operative necessity of a straight catheterization, or a consult to urology for urinary retention. The most commonly recorded intraoperative medications were analyzed. Student's t-test, Fisher exact t-test, and the Wilcoxon rank-sum test were used to compare the use and dose-response of intraoperative medications between patients with and without POUR. A multivariate analysis was performed to analyze the differential effects of potential risk factors for developing POUR.

Results: A total of 205 patients were included in the study; of which, $17 \%$ experienced POUR $(n=34)$. Patients with POUR had an increased duration of hospitalization. Of the commonly utilized intraoperative anesthetic medications, administration of phenylephrine and neostigmine were associated with POUR (phenylephrine, $32.3 \%$ vs. $13.8 \%, p=0.017$; neostigmine, $19.5 \%$ vs. $6.5 \%, p=0.042)$. When testing for the effects of medication dosage on POUR, parametric analysis demonstrated an association of increasing dose of neostigmine with POUR (4.66 vs. $4.22 \mathrm{mg}, \mathrm{p}$ $=0.023$ ). A nonparametric analysis demonstrated an association of increasing doses of both neostigmine and phenylephrine with POUR (neostigmine, 4.25 vs. $3.16 \mathrm{mg}, \mathrm{p}=0.02$; phenylephrine, 105.88 vs. $40.64 \mathrm{mg}, \mathrm{p}=0.008)$. Multivariate analysis identified neostigmine, phenylephrine, and benign prostatic hyperplasia (BPH) as the three strongest individual risk factors for developing POUR.

Discussion/Conclusion: Approximately $20 \%$ of patients may develop POUR following routine lumbar spine surgery. The use of certain intraoperative anesthetics such as phenylephrine and neostigmine are strongly associated with the development of POUR. This finding suggests that there may be modifiable anesthetic risk factors to prevent the development of POUR in patients undergoing lumbar spine surgery. Future prospective, controlled studies specifically addressing these findings could lead to improved patient care and decreased healthcare costs.

Paper \#10. ASA Class as an Independent Risk Factor for Complications After Adult Spinal Deformity Surgery

Sulaiman Somani, $B S^{1}$, Joung Heon Kim, BS , John Di Capua, MHS, BS $S^{1}$, Parth Kothari, BS ${ }^{1}$, Nathan Lee, BS $S^{1}$, Samuel Cho, MD

${ }^{1}$ Icahn School of Medicine at Mount Sinai, New York, New York, ${ }^{2}$ Icahn School of Medicine at Mount Sinai, New York, NY, ${ }^{3}, \mathrm{NY}, \mathrm{NY}$

Background/Introduction: The American Society of Anesthesiologists (ASA) classification system is the current paradigm in assessing a patient's preoperative health. This study seeks to understand the ASA classification system's association with malicious postoperative outcomes following elective adult spinal deformity surgery (ADS).

Materials/Methods: A retrospective cohort analysis was performed on the prospectively collected American College of Surgeons National Surgical Quality Improvement Program (ACS NSQIP) database from 2010 to 2014 . Patients ( $\geq 18$ years of age) undergoing ADS were divided into cohorts based on whether their ASA classification was $\geq 3$. Chi-square- and t-tests were used to compare the demographic, surgery-specific, preoperative comorbidity, and postsurgical complications for both groups. Multivariate logistic regression models were generated to identify independent risk factors. 
Results: A total of 5,803 patients met the inclusion criteria, where $3,087(53.2 \%)$ of the patients were classified as ASA $\geq 3$. Multivariate logistic regressions revealed ASA class $\geq 3$ to be an independent risk factor for the following complications: mortality $(\mathrm{OR}=20.95(2.84,154.38), \mathrm{p}=0.0028)$, length of stay $\geq 5$ days $(\mathrm{OR}=1.69(1.48,1.94), \mathrm{p}<0.0001)$, any complications $(\mathrm{OR}=$ $1.37(1.19,1.57), \mathrm{p}<0.0001)$, wound complications $(\mathrm{OR}=1.76$ $(1.21,2.56), \mathrm{p}=0.0030)$, pulmonary complications $(\mathrm{OR}=2.28$ $(1.56,3.33), \mathrm{p}<0.0001)$, UTI (OR = $1.59(1.06,2.39), \mathrm{p}=0.0024)$, cardiac complication $(\mathrm{OR}=3.71(1.52,9.08), \mathrm{p}=0.0041)$, transfusion $(\mathrm{OR}=1.27(1.10,1.47), \mathrm{p}=0.0009)$, sepsis $(\mathrm{OR}=$ $2.70(1.68,4.33), \mathrm{p}<0.0001)$, and reoperation within 30 days of surgery $(\mathrm{OR}=1.60(1.18,2.16), \mathrm{p}=0.0023)$.

Discussion/Conclusion: ASA class can serve as a major indicator of potential postoperative complications, including mortality, following elective ADS

Paper \#11. Effect of Surgical Setting (Tertiary versus Community Hospitals) on Hospital Reported Outcomes for Degenerative Elective Lumbar Spinal Procedures

Neil Sardesai, MD, Ehsan Jazini, MD, Tristan Weir, BS, Eugene Koh, MD, PhD, Kelley Banagan, MD, Daniel Gelb, MD, Steven Ludwig, MD

\section{, Baltimore, MD}

Background/Introduction: As hospital compensation becomes increasingly dependent on pay-for-performance and bundled payment compensation models, hospitals seek to reduce costs and improve patient outcomes by decreasing patient length of stay (LOS), potentially avoidable utilizations (PAUs: readmissions/ revisits), and in-hospital provider preventable complications (PPCs - as defined by CMS). Large national databases typically provide such metrics, but are unreliable and lack clinical detail. There is also a lack of data comparing outcome measures between large tertiary centers and community centers. We sought to evaluate hospital-reported outcomes measures - LOS, PAUs, and PPCs for elective lumbar procedures at tertiary versus community (TvC) centers within the same hospital system. We hypothesize that no difference in LOS, PPCs, and PAUs exists between tertiary and community hospitals.

Materials/Methods: A physician-driven, prospective database of a single medical system consisting of one tertiary and four community hospitals was retrospectively evaluated for the three most common lumbar surgeries: laminectomy, laminectomy with fusion, and microdiscectomy. 431 consecutive adults who underwent one of these procedures were evaluated from January through July 2015. The primary outcome was LOS, and secondary outcomes were PPCs (during hospital stay) and PAUs (30 day allcause readmission/revisit) for $\mathrm{TvC}$ facilities. Independent variables included: age, severity of illness (SOI), surgical procedure, and surgical specialty (orthopaedic or neurosurgery).

Results: Univariate analysis demonstrated that LOS was significantly greater in tertiary $(3.7 \pm 4.3$ days $)$ versus community $(2.2 \pm 1.9$ days $)$ facilities $(\mathrm{p}=0.0003$, Table 1$)$. Multivariate regression analysis revealed surgical facility $(\mathrm{p}<0.001)$ significantly affects LOS independent of age, discharge disposition, SOI, and procedure. We found no correlation between surgical facility and PPCs, but a trend existed towards higher PAUs in community versus tertiary facilities $(3.2 \%$ versus $0 \%, \mathrm{p}=0.068)$. Higher PPCs were significantly associated the procedure performed, SOI, and discharge disposition. Surgical specialty was not associated with PPC rates.

Discussion/Conclusion: While tertiary centers have a significantly longer LOS, there is a trend towards higher revisits/ readmission rates in community facilities. There is no association between tertiary versus community hospitals and rates of PPCs. These findings suggest a tradeoff between LOS and revisit/ readmissions. This surgeon driven data can help develop more effective protocols to decrease LOS while minimizing PAUs and PPCs.

Paper \#12. Predicting Discharge Disposition After Lumbar Fusion

Ashley Rogerson, $M D^{1}$, Jenis Louis, $M D^{2}$, Andrew Mason, BS, $M S^{3}$, Jonna Capecci, $P T^{4}$, David Tybor, $P h D, M P H^{5}$

${ }^{1}$ Newton Wellesley Hospital, South Boston, MA, ${ }^{2}$ Massachusetts General Hospital, Boston, MA, ${ }^{3}$ Tufts University School of Medicine, Boston, MA, ${ }^{4}$ Newton Wellesley Hospital, Newton , MA, ${ }^{5}$ Tufts University, Boston, MA

Background/Introduction: Annual rate and cost of lumbar spine fusion are increasing. Discharge disposition after elective inpatient surgery has an effect on overall cost and length of stay (LOS) after surgery. Oldmeadow et al. developed the Risk Assessment and Predictor Tool (RAPT) to identify patients that are high risk for discharge to rehab facility after total joint arthroplasty. Our goal was to develop a similar scoring tool that can be administered before patients undergo lumbar spine fusion.

Materials/Methods: Retrospective review of 300 patients undergoing lumbar fusion was performed and patient demographic, intraoperative and postoperative data were collected. Patients discharged to rehabilitation facility were compared to those discharged home to determine which variables were significantly different between the groups. Variables that could be collected preoperatively were used to create a logistic regression analysis model. Balancing ease of use with predictive accuracy of each variable, the Preoperative Discharge Assessment Tool (PDAT) was developed. This tool was then tested on the patient cohort to determine its internal predictive accuracy. Significance was set at $\mathrm{p}<0.05$.

Results: 227 patients (76\%) were discharged home and 73 patients $(24 \%)$ were discharged to a rehab facility. Median LOS was $3 \pm 1$ days for all patients. Variables included in the PDAT were American Society of Anesthesiologists physical status (ASA) classification, number of levels fused, home living situation (alone or with others), and preoperative assistive device (AD) use. Predictive accuracy of the PDAT was $85.5 \%$. Fifteen patients requested to be discharged to rehab and 14 (93.3\%) of those patients were ultimately discharged to rehab. These patients were older (67.9 vs 58.5 years) and more often lived alone (46.2\% vs $7.0 \%)$.

Discussion/Conclusion: Patients discharged to rehab were tended to be older ( 68.3 vs 56.0 years), have higher a higher BMI (31 vs 29$)$, live alone (20\% vs $5.3 \%$ ) and undergo larger operations. Our scoring tool uses preoperative living situation, AD use, number of levels fused and ASA classification to predict discharge destination. It has similar predictive properties to the RAPT. Patient preference for a discharge to rehab should be taken into consideration as well. 
Paper \#13. Predictors of Discharge to a Non-Home Facility in Patients Undergoing Lumbar Decompression without Fusion for Degenerative Spine Disease: An ACS-NSQIP Study

Meghan Murphy, M.D. ${ }^{1}$, Patrick Maloney, M.D. ${ }^{2}$, Brandon McCutcheon, M.D. ${ }^{2}$, Panagiotis Kerezoudis, M.D. ${ }^{2}$, Daniel Ubl, B.A. ${ }^{3}$, Cynthia Crowson, M.S. ${ }^{3}$, Mohamad Bydon, M.D. ${ }^{4}$

${ }^{1}$ Mayo Clinic, Rochester, Minnesota, ${ }^{2}$ Mayo Clinic, Rochester, $\mathrm{MN},{ }^{3}$ Mayo Clinic, Rochester , MN,${ }^{4}$, Rochester, $\mathrm{MN}$

Background/Introduction: Patients recovering from decompressive laminectomy without fusion may require assistance with activities of daily living and physical/occupational therapy upon hospital discharge. Using a national surgical registry, the premorbid status and perioperative characteristics of patients undergoing lumbar decompression were examined for associations with discharge status.

Materials/Methods: The 2011-2013 American College of Surgeons National Surgical Quality Improvement Program(ACSNSQIP) data was used for this retrospective cohort analysis. Patients admitted from home with degenerative spine disease for lumbar decompression without fusion were included. Thirtyday outcomes and operative characteristics were compared as a function of patient discharge using chi-square and Kruskal-Wallis tests. Multivariable logistic regression was used to investigate factors that were independently associated with discharge to a non-home facility.

Results: Patients $(\mathrm{N}=8627)$ were stratified into two groups by discharge disposition, home versus non-home facility. On unadjusted analysis, dismissal to a non-home facility was significantly associated with increased age, steroid use for chronic condition, hypertension requiring medication, chronic kidney disease requiring dialysis, severe COPD, smoking status within one year, BMI, dependent preoperative functional status, ASA score, and $3+$ levels of decompression(all p<0.001). On multivariable analysis, age ( $85+$ vs $<65$, OR $13.57,95 \%$ CI: 9.47-19.49), number of levels of decompression(3+ vs 1, OR 1.75, 95\% CI: 1.40-2.20), African American race vs Non-Hispanic White(OR 1.87, 95\% CI: 1.39-2.51), BMI(40+vs 18.5-24.9, OR 1.74, 95\% CI: 1.23-2.47), ASA score(4 vs <3, OR 2.35, 95\% CI: 1.56-3.55), hypertension requiring medications(OR $1.29,95 \% \mathrm{CI}: 1.06-1.57)$, dependent functional status(OR 3.92, 95\% CI: 2.71-5.67), diabetes(OR $1.47,95 \%$ CI: 1.22-1.76), smoking(OR 1.40, 95\% CI: 1.10-1.9), glomerular filtration rate(GFR) $\left(<15\right.$ vs $\geq 90 \mathrm{~mL} / \mathrm{min} / 1.73^{\wedge} 2$, OR $4.17,95 \%$ CI: $1.48-11.78)$, hematocrit $(<35$ vs $35+$, OR $1.76,95 \%$ CI: $1.36-2.28), \operatorname{INR}(\geq 1.3$ vs $<1.3$, OR $2.32,95 \%$ CI: $1.38-3.87)$, and operative time( $3+$ hours vs $<1$ hour, OR $5.34,95 \%$ CI: $3.74-$ 7.64) were independently associated with an increased odds of discharge to non-home facilities.

Discussion/Conclusion: Particular premorbid status markers and operative course variables can influence the discharge disposition in lumbar decompression patients. Identifying specific factors that contribute to a greater likelihood of dismissal to skilled facility or rehab placement can further inform both surgeons and patients during preoperative counseling and disposition planning.

Paper \#14. Readmission Rates, Reasons, and Risk Factors in Elderly Patients Treated with Lumbar Fusion for Degenerative Pathology

Varun Puvanesarajah, BS ${ }^{1}$, Hamid Hassanzadeh, $M D^{1}$, Adam Shimer, $M D^{1}$, Francis Shen, $M D^{2}$, Anuj Singla, $M D^{2}$

\section{${ }^{1}$, Charlottesville, VA, ${ }^{2}$, Charlottesville, Virginia}

Background/Introduction: Early post-operative readmissions following spine surgery represent a significant source of increased cost and morbidity. As the elderly population represents a demographic with a growing need for spine surgery, readmissions within this population is of significant interest. The objectives of this study were to determine readmission rates following 1-2 level, primary, elective lumbar spinal fusion surgery for degenerative pathology and elucidate risk factors that predict increased risk of thirty day readmission.

Materials/Methods: Medicare data (2005 to 2012) from an insurance database was queried for patients who underwent primary 1-2 level posterolateral lumbar spine fusion surgeries for degenerative lumbar pathology. After applying specific exclusion criteria to select for elderly patients (65-84 years) undergoing mostly elective procedures, 52,567 patients formed the final study population. Readmission rates for medical, surgical, and all reasons were calculated within thirty days, ninety days, and one year post-operatively. Risk factors for medical, surgical, and all thirty-day readmissions were also determined.

Results: Within thirty days, ninety days, and one year, 1,510 $(2.9 \%), 2,776(5.3 \%)$, and 6,574 (12.5\%) patients were readmitted, respectively. At thirty days, surgical diagnoses constituted $50.1 \%$ of all readmissions. This decreased to $44.8 \%$ and $31.8 \%$ at ninety days and one year, respectively. Common medical reasons for readmission were of cardiac, gastrointestinal, and pulmonary origin (Figure 1A). Wound infection was the reason for readmission in $25.8 \%$ of all readmissions within thirty days (Figure 1B). Diagnoses of chronic pulmonary disease (OR 1.41 $95 \%$ CI $1.22-1.63$ ), obesity (OR $2.2095 \%$ CI $1.90-2.54$ ), and positive smoking history (OR $1.3395 \%$ CI 1.15 - 1.54) were associated with increased risk of surgical readmission.

Discussion/Conclusion: Elderly patients undergoing lumbar spine fusion experience 30-day, 90-day, and 1-year readmission rates of $2.9,5.3$, and $12.5 \%$ for both medical and surgical reasons. Surgical site infection and wound complications are among the most common surgery-related reasons for readmission. Medical diagnoses are more predominant during later readmissions, highlighting the comorbidity burden present in elderly patients.

Paper \#15. Spine Surgical Invasiveness Index is a Predictor for Readmission

Ashley Rogerson, $M D^{l}$, Jenis Louis, $M D^{2}$, Andrew Mason, BS, $M S^{3}$, Jonna Capecci, $P^{4}$

${ }^{1}$ Newton Wellesley Hospital, South Boston, MA, ${ }^{2}$ Massachusetts General Hospital, Boston, MA, ${ }^{3}$ Tufts University School of Medicine, Boston, MA, ${ }^{4}$ Newton Wellesley Hospital, Newton , MA

Background/Introduction: Surgical invasiveness index (SII) was defined by Mizra et al. in 2008. SII has since been used to correlate increasing scores with a higher risk of postoperative surgical site infection requiring return to the operating room (Cizik et al, 2012). Our goal was to examine whether an increase in SII was associated with an increased risk of 90 day readmission after any lumbar spine surgery.

Materials/Methods: All patients undergoing lumbar spine surgery at a single community-based academic hospital from January 1 to December 31, 2014 were reviewed. SII was calculated for each surgery based on the dictated operative report. Readmissions are tracked by the institution quality and safety database. Wilcoxon Rank-Sum test was used to assess the difference in mean SII for readmitted patients compared with those who were not readmitted. Logistic regression analysis was used to test the association of SII with 90-day readmission. Due to strong association of BMI with readmission risk, BMI > 35 was included in a multivariate logistic regression model. 
Results: SII was calculated for 626 patients. There were 19 readmissions within 90 days of surgery. Nine patients had postop infections, 4 patients had a wound complication, 4 patients had hardware complication, and 4 had a medical readmission. Two patients had both a medical readmission and hardware complication. Median SII for all patients was 3 (IQR=1-10). Median SII for patients who were readmitted was $8(\mathrm{IQR}=4-12)$ compared with a mean of $3(\mathrm{IQR}=1-10)$ for those who were not readmitted $(\mathrm{p}<0.05)$. RR of readmission was $5.41(\mathrm{p}=0.026)$ for the high invasiveness group compared with the low invasiveness group $(\mathrm{p}<0.05)$. BMI $>35$ is also a significant predictor of readmission with a RR of $3.61(p=0.005)$. The high invasiveness group remained significant as a predictor of readmission when BMI was included in logistic regression model.

Discussion/Conclusion: Planning a safe discharge are spinal surgery is imperative. Close attention should be devoted to patients undergoing surgery with a high SII. This information can be useful in caring for the patient during their hospital stay and coordinating discharge. Patients with a SII $>8$ are at increased risk for 90 day readmission.

Paper \#16. The Effect of Vancomycin Powder on the Rates of Infection and Pseudarthrosis in Lumbar Spine Surgery; A Retrospective Analysis of 532 Patients

Sukanta Maitra, $M D^{l}$, Jordan Kump, $B S^{1}$, Yue Zhang, $P h D^{2}, W$. Ryan Spiker, $M D^{3}$, Brandon Lawrence, $M D^{3}$, Darrel Brodke, $M D^{3}$

${ }^{1}$ University of Utah, Salt Lake City, UT, ${ }^{2}$ University of Utah, Salt lake City, Utah, ${ }^{3}$, Salt Lake City, Utah

Background/Introduction: Surgical site infection (SSI) represents a major complication in spine surgery. The application of topical vancomycin powder has been shown to effectively reduce SSI's. Recent in vitro studies have suggested that topical application of lyophilized vancomycin has an inhibitory effect on osteoblast proliferation and differentiation that may adversely affect fusion rates. The primary purpose of this study was to examine our institution's overall revision rate before and after the routine use of vancomycin powder in patients undergoing spinal fusion. Our secondary goal was to determine the infection rate prior to and after the initiation of vancomycin powder into the wound.

Materials/Methods: A retrospective chart review including all patients undergoing lumbar fusion with at least 2 years of clinical follow up. The pre-vancomycin group $(1 / 1 / 2007-12 / 31 / 2009)$ were compared to the vancomycin group $(1 / 1 / 2012-12 / 31 / 2013)$ for development of a SSI requiring debridement, return to OR for revision of the prior surgery and data including number of levels fused, use of bone morphogenetic protein (BMP), and use of interbody grafts was recorded.

Results: A total of 273 patients were identified in the prevancomycin group and 259 in the vancomycin group. Overall infection rate was $10 / 259(3.9 \%)$ in the pre-vancomycin group and $6 / 273(2.2 \%)$ in the vancomycin group. There was a significant difference in the use of interbody grafts $(p=0.016)$ and BMP use in the pre-vancomycin group $(\mathrm{p}=0.001)$. However, no significant difference was noted in levels fused or revision rates between the two groups.

Discussion/Conclusion: The use of topical vancomycin powder did not significantly alter our fusion or revision rates despite significantly less use of inter-body grafts and BMP in our Vancomycin cohort. Further in vivo and in vitro studies are necessary to fully elucidate vancomycin powder's role in the maturation of a fusion.
Paper \#17. Occult Infection Demonstrated in Patients Who Have Undergone Spinal Surgery

Tucker Callanan, $B S^{l}$, Darren Lebl, $M D^{2}$, Andrew Sama, $M D^{2}$, Federico Girardi, $M D^{3}$, Sam Miller, $B S^{4}$, Frank Cammisa, $M D^{2}$, Celeste Abjornson, $\mathrm{PhD}^{2}$

${ }^{1}$, New York, NY, ${ }^{2}$ Hospital for Special Surgery, New York, New York, ${ }^{3}$, New York, New York, ${ }^{4}$, Providence, RI

Background/Introduction: Often in revision orthopedic surgery, chronic pain and/or pseudoarthrosis are the main indications, and normal screening rules out the presence of infection. If insidious organisms exist in low concentrations in a biofilm that surrounds the majority of implant surfaces, these organisms can disrupt bone formation leading to micro-motion (pain generation) or failure of a solid fusion. A previous study demonstrated that patients with chronic low back pain that were provided antibiotic treatment showed significant improvement compared to a placebo group, suggesting that occult infection could be a factor. The purpose of this investigation was to ascertain the incidence of occult pathogens in revision spinal surgery.

Materials/Methods: Forty-one consecutive patients undergoing a posterior revision requiring removal of pedicle screw implementation were studied. Swabs from each screw hole and around each screw head were taken for long cultures (14 days). The hardware was catalogued and analyzed for wear. Cultured samples were embedded for histological examination to quantify the existence of colonies of bacteria in proximity to the implant. A regression analysis was performed to find the relationship of variables collected to determine predictive indicators of occult pathogens.

Results: Occult bacteria were found in $37 \%(15 / 41)$ of the tissue surrounding the implants of patients undergoing spine revision surgery. The most prevalent bacteria found were $P$. acnes, in $47 \%(7 / 15)$ of infected patients. Bacteria took on average five days to grow out on culture plates. There was no significant difference in the average length between index and revision procedures between patients with positive cultures (4.8 years) and patients with negative cultures (4.4 years). There was also no direct correlation between implant parameters or surgical location, however males showed a significantly higher incidence of positive culture results than females ( $p$-value $<0.05$ ).

Discussion/Conclusion: Identifying the factors that contribute to failed surgery and/or chronic pain are critical. The results of this study demonstrate that occult infections could be a contributing factor to this issue. The study also suggests that long term cultures (fourteen days) are vital to identifying these pathogens. Finally, the findings suggest that males were more susceptible to occult infections then women. 
Paper \#18. Perioperative Complications Do Not Affect Patient Satisfaction Following Adult Spinal Deformity Surgery

Farbod Rastegar, $M D^{\prime}$, D. Hamilton, $M D^{2}$, Jayme Hiratzka, $M D^{l}$, Shannon Hiratzka, MPH ${ }^{1}$, Justin Smith, MD ${ }^{3}$, Shay Bess, $M D^{4}$, Justin Scheer, $B S^{5}$, Alan Daniels, $M D^{6}$, Virginie Lafage, PhD ${ }^{7}$, Robert Hart, M.D. ${ }^{8}$

'Oregon Health \& Science University, Portland, Oregon, ${ }^{2}$ University of Pittsburgh Medical Center, Pittsburgh, PA, ${ }^{3}$ University of Virginia, Charlottesville, VA, ${ }^{4} \mathrm{NYU}$ Hospital for Joint Diseases, White Plains, NY, ${ }^{5}$ University of California San Diego, La Jolla, CA, ${ }^{6}$ Department of Orthopaedics, Division of Spine Surgery- Adult Spinal Deformity Service, Warren Alpert Medical School of Brown University, Providence, Rhode Island, ${ }^{7}$ Hospital for Special Surgery, New York, NY, ${ }^{8}$ Oregon Health and Science University, Portland, OR

Background/Introduction: Patient satisfaction after adult spinal deformity surgery remains incompletely assessed compared to radiographic and clinical outcomes. The goal of this study was to evaluate the change in patient satisfaction after adult thoracolumbar deformity surgery in patients experiencing perioperative complications.

Materials/Methods: A prospective multicenter cohort of 256 patients undergoing thoracolumbar fusion for adult spinal deformity was analyzed retrospectively at minimum 2-year follow-up. Complications were defined as no, minor, major, or any (major + minor). Clinical satisfaction was assessed using Scoliosis Research Society-22- (SRS-22) satisfaction scores, which allows a comparison to satisfaction with pre-operative treatment. Comparisons in satisfaction were made at 6 weeks, 1 year, and 2 year follow up for patients experiencing no, minor, or major, or any complications.

Results: $112(44 \%)$ patients experienced a complication (58 major and 54 minor) within the first 6 weeks after surgery. For patients with no, minor, major, or any complications, there was significant improvement in SRS-satisfaction compared to baseline at 6 weeks, 1 year and 2 years $(p<0.0001$ for all groups). There was no significant change in patient satisfaction from 6 weeks to 2 years for any group $(\mathrm{p}=0.337$ any complication, $\mathrm{p}=0.306$ major, $\mathrm{p}=0.741$ minor). There was no difference in satisfaction between patients with no, minor, major, or any complication at any postoperative time point (Figure 1).

Discussion/Conclusion: Patients undergoing thoracolumbar fusion for adult deformity expressed increased satisfaction with treatment over pre-operative state by 6 weeks post-operatively, and maintained similar levels of satisfaction out to 2 years postoperatively. Perioperative complications do not seem to have a substantial effect on overall patient satisfaction. Determinants of patient satisfaction may be related to other factors, such as preoperative expectations, inpatient experience and relationship with the surgeon.

Paper \#19. Complications of Spine Surgery: Factors Predictive of Malpractice Litigation

Roy Ruttiman, $\mathrm{MS}^{1}$, Adam Eltorai, BAㄹ, J. Mason DePasse, $M D^{3}$, Bielinsky Brea, $B S^{l}$, Mark Palumbo, $M D^{4}$, Alan Daniels, $M D^{5}$

${ }^{1}$, Providence, RI, ${ }^{2}$ Warren Alpert Medical School of Brown University, Providence, RI, ${ }^{3}$ Department of Orthopaedics, Warren Alpert Medical School of Brown University, Providence, Rhode Island, ${ }^{4}$ Department of Orthopaedics, Division of Spine Surgery, Warren Alpert Medical School of Brown University , Providence, RI, ${ }^{5}$ Department of Orthopaedics, Division of Spine Surgery-
Adult Spinal Deformity Service, Warren Alpert Medical School of Brown University, Providence, Rhode Island

Background/Introduction: Major complications related to spine surgery often lead to litigation. Few studies have evaluated the association between spine surgical complications and medical malpractice proceedings, outcomes and awards.

Materials/Methods: A search for "spine surgery" utilizing the medicolegal research service VerdictSearch (ALM Media Properties, LLC, New York, NY) was conducted spanning February 1988 to May 2015. The age and sex of the patient, type of surgical complication, and the medical specialty of provider were recorded. Complications were sorted into catastrophic and non-catastrophic categories. Catastrophic complications were defined as paralysis, anoxic/hypoxic brain injury, and death; noncatastrophic complication included all other complications. Chi squared and t-tests were utilized to evaluate the effect of these variables on case outcomes and awards granted

Results: A total of 569 legal cases were examined; 335 cases were excluded due to irrelevance or insufficient information. Of the 234 cases available for review, 28\% (66/234) involved catastrophic complications with $72 \%$ related to non-catastrophic complications (Table 1). Overall, 54.2\% (115 cases) resulted in a defendant ruling, 26.1\% (58) resulted in a plaintiff ruling, and $19.7 \%$ (42) resulted in settlement. Total liabilities of the 234 cases were $\$ 335,550,287$. The award granted for the plaintiff rulings ranged from $\$ 134,000$ to $\$ 38,323,196$ (mean $\$ 4,045,205 \pm$ $\$ 6,804,647$ ) while the award for settlements ranged from $\$ 125,000$ to $\$ 9,000,000$ (mean $\$ 1,930,278 \pm \$ 2,113,593$ ) (Table 2). In cases with a plaintiff ruling, catastrophic complications resulted in awards significantly larger than non-catastrophic complications (\$6.1 million vs $\$ 2.9$ million; $\mathrm{p}<0.04$ ). In cases resulting in settlement, there was no statistical difference between awards granted for catastrophic complications and non-catastrophic complications ( $\$ 2.4$ million vs $\$ 1.5$ million; $p<0.09$ ). In cases of catastrophic complications, physicians were more likely to lose in court $(66.7 \%$ vs $37.5 \% ; \mathrm{p}<0.001)$ and to settle out of court $(33.3 \%$ vs $14.3 \% \mathrm{p}<0.001)$ compared to non-catastrophic complication cases. The medical specialty of provider and the age and sex of the patient were not statistically associated with case outcome or award granted.

Discussion/Conclusion: Catastrophic complications following spine surgery are a predictor of medicolegal case outcome in favor of the plaintiff and are linked to large sums awarded to the plaintiff.

\section{Paper \#20. Bioskills Laboratory for Resident Education}

\author{
Barrett Boody, M.D. ${ }^{1}$, Jason Savage, M.D. ${ }^{2}$, Wellington Hsu, \\ $M . D .{ }^{3}$
}

${ }^{1}$ Northwestern Memorial Hospital, Chicago, illinois, ${ }^{2}$ The Cleveland Clinic, Cleveland, Ohio, ${ }^{3}$ Northwestern Memorial Hospital, Chicago, Illinois

Background/Introduction: Educating young surgeons in spine surgery is a difficult task due to the work hour restrictions placed on residents as well as the high-stakes nature of the surgical procedures. We propose that a brief bioskills laboratory would improve residents' performance and understanding of open lumbar decompressions.

Materials/Methods: 20 orthopaedic residents and medical students were enrolled in the study and randomized to control $(n=9)$ or intervention $(n=11)$ groups based on level of experience (medical students, junior resident, senior resident). A pre-test and post-test sawbones lumbar laminectomy on a degenerative lumbar stenosis sawbones model was performed by each participant 
and evaluated by a blinded observer for subjective performance (Objective structured assessment of technical skills, OSATS) and objective completion of decompression. Prior to the post-test, the intervention group underwent a 40-minute educational module consisting of a powerpoint presentation and hands-on sawbones practice with focused feedback on technical skills and steps of the procedure. Following the post-test, participants self rated their technical skills and knowledge of the procedure (Physician Performance Diagnostic inventory scale, PPDIS).

Results: Results were analysed using the Wilcoxon T-test to account for limited sample sizes and variability in reported scores. The intervention group yielded a significant improvement in overall OSATS (possible scores 5-25, mean improvement 6.3 vs $0.9, p=0.0337$ ) and PPDIS (possible scores 5-25, mean improvement 5.5 vs $0.1, \mathrm{p}=0.0016$ ) scores. While the objective decompression scores improved in the intervention group, this did not reach significance (possible scores 6-24, mean improvement 6.4 vs $2.2, \mathrm{p}=0.0733$ ). Subgroup analyses based on level of experience is limited due to small sample sizes.

Discussion/Conclusion: A brief lumbar laminectomy bioskills laboratory can be useful resident and student educational tool, leading to significantly improved scores in residents' subjective performance and trending toward improvement in objective grading of completeness of decompression. Power analyses for this project are unclear, since the size of anticipated effect has not been reported previously in the literature. While our study is limited to 20 participants due to funding, we believe that the statistically significant observed improvement in participant skills is clinically relevant and that similar laboratories can be powerful educational tools for surgeons in training.

Paper \#21. The Utility of Routine Hematologic Values Following Elective Posterior Lumbar Instrumented Spinal Fusion

Daniel Baluch, $\mathrm{MD}^{\prime}$; Christopher O'Boynick, $M D^{2}$, Daniel Dixon, MD ${ }^{3}$, Bruce Darden, $\mathrm{MD}^{3}$, Susan Odum, PhD ${ }^{4}$, Leo Spector, $M D^{3}$

${ }^{1}$ OrthoCarolina Spine Center, Charlotte, NC, ${ }^{2}$ Premier Care Orthopedics \& Sports Medicine, St. Louis, MO, ${ }^{3}$ OrthoCarolina, Charlotte, NC, ${ }^{4}$ OrthoCarolina Research Institute, Charlotte, NC

Background/Introduction: Background: With advances in surgical techniques and perioperative management, the need for postoperative blood transfusion after spinal arthrodesis has decreased. Currently there are no evidence-based guidelines for ordering routine postoperative hemoglobin levels after posterior lumbar instrumented fusion. The utility of routine post-op hemoglobin levels has been evaluated in other surgeries and not been shown to improve patient care. This study evaluates the utility of routine hematologic testing in this patient population.

Materials/Methods: Methods: A retrospective chart review was performed on 368 patients who underwent elective posterior spinal instrumented fusion at our institution over a five-year period. Exclusion criteria included age under 18 years, anterior or lateral procedures, thoracic instrumentation, trauma, and absence of pre- or postoperative hemoglobin levels. 292 patients met the inclusion criteria. The number of levels fused ranged from one to four. Patients were grouped by age as less than or greater than 65 years. An age and gender-adjusted receiver operator curve was used to determine the area under the curve (AUC), or precision of predicting transfusion, using preoperative hemoglobin thresholds of $11 \mathrm{~g} / \mathrm{dL}, 12 \mathrm{~g} / \mathrm{dL}$, and $13 \mathrm{~g} / \mathrm{dL}$.

Results: Results: Of the 292 patients, 22 (7.5\%) received a postoperative blood transfusion. Transfused patients were more likely to be female $(11 \%$ vs. $3 \%$; $=0.01)$ and had significantly lower average preoperative hemoglobin levels $(12.0 \mathrm{~g} / \mathrm{dL}$ vs. $13.6 \mathrm{~g} / \mathrm{dL}, \mathrm{p}<0.0001)$. Neither age, estimated blood loss, use of an intraoperative autologous blood transfusion device, nor the reinfusion volumes were significantly associated with the need for transfusion. Using the preoperative hemoglobin thresholds of $11 \mathrm{~g} / \mathrm{dL}, 12 \mathrm{~g} / \mathrm{dL}$, and $13 \mathrm{~g} / \mathrm{dL}$, the decision of whether or not to transfuse would be correct $72 \%, 82 \%$, and $80 \%$ of the time, respectively [AUC $0.72(95 \%$ CI .61-.83), AUC 0.82 (95\% CI .73-.91), AUC 0.80 (95\% CI .72-.87)].

Discussion/Conclusion: Conclusions: The transfusion rate for our study was $7.5 \%$. Transfused patients tended to be female and had significantly lower starting hemoglobin values. These findings suggest that a preoperative hemoglobin value of $12 \mathrm{~g} / \mathrm{dL}$ may be a reasonable threshold that can be used as a guide for ordering routine postoperative hemoglobin levels and preoperative type and cross.

Paper \#22. Results of LSRS Membership Questionnaire Regarding Fusion Surgery for Lumbar Stenosis and Spondylolisthesis

David Kim, $M D^{1}$, Kadimcherla Praveen, $M D^{2}$, Kevin Baker, $P h . D^{3}$, Jeffrey Fischgrund, $M D^{3}$

${ }^{1}$ New England Baptist Hospital, Boston, MA, ${ }^{2}$, Jersey City, NJ, ${ }^{3}$, Royal Oak, Michigan

Background/Introduction: Lumbar degenerative spondylolisthesis is the most common diagnosis leading to spinal fusion surgery in the United States. Over the past several years, marked changes in practice patterns have occurred in terms of surgical technique and use of bone graft alternatives.

Materials/Methods: A survey was distributed among the spine surgeon attendees of the 2014 LSRS annual meeting to determine common practice for treatment of patients with degenerative spondylolisthesis/stenosis (primary, non-revision single level).

Results: Among 61 eligible member attendees, 37 returned completed questionnaires (60.7\% response rate). The responses were compiled and the following average rates were reported. Over the preceding year, surgeons fused $81 \%$ of patients with a grade 1 slip (range $10-100 \%$ ). $91 \%$ of fusions were performed with instrumentation $(0-100 \%)$. $51 \%$ of fusions were performed with an interbody technique (0-100\%). For grade 2 slips, the following averages were reported: $96 \%$ of patients fused (25-100\%), 94\% of fusions were instrumented (5-100\%), 66\% of fusions included interbody technique (0-100\%). In terms of posterolateral bone graft options, $100 \%$ of surgeons routinely used local bone graft with or without extenders, $5.9 \%$ BMP, $17.6 \%$ ceramic composite, $17.6 \%$ DBM, 8.8\% iliac crest autograft, $14.7 \%$ bone marrow aspirate, $32.4 \%$ corticocancellous allograft chips. $23.5 \%$ of surgeons used solely local bone. For the interbody space, $45.2 \%$ of surgeons used only local bone in addition to the cage, $16.1 \%$ ceramic composite, $11.8 \%$ iliac crest autograft, $9.8 \%$ DBM, 5.9\% corticocancellous allograft chips, $5.9 \%$ BMP, $2.9 \%$ bone marrow aspirate. $90.5 \%$ used PEEK cages, 5.9\% structural allograft, $4.8 \%$ carbon fiber and $4.8 \%$ titanium cages. $88.2 \%$ of surgeons routinely remove cartilage from facet joints and $64.7 \%$ place graft in the joint. $61.8 \%$ believe bilateral facet joint fusion is clinically equivalent to posterolateral fusion in terms of long-term outcome, while $17.6 \%$ believe unilateral facet joint fusion is equivalent.

Discussion/Conclusion: There is a broad range of practice patterns even among a select group of lumbar spine surgical specialists with respect to treatment for single-level spondylolisthesis/stenosis. The overwhelming majority of surgeons perform instrumented fusion, and most include interbody fusion. There is even greater variation in bone graft selection with heavy reliance on local autograft despite recent evidence suggesting poor fusion rates with use of only local bone graft. 
Paper \#23. The Utility of EMG Stimulation Compared to Intraoperative Imaging With the O-Arm for Evaluating Pedicle Screw Breaches: A Prospective Study of 1006 Screws in 164 Cases

Ricky Kalra, M.D. ${ }^{1}$, Erica Bisson, MD, MPH ${ }^{2}$, Meic Schmidt, $M D, M B A^{2}$; Andrew Dailey, $M D^{2}$

\section{${ }^{1}$, Salt Lake City, UT, ${ }^{2}$ University of Utah, Salt Lake City, UT}

Background/Introduction: Historically, intraoperative stimulus-evoked electromyography (EMG) has been utilized to detect pedicle screw breaches. The use of intraoperative O-arm imaging to assess pedicle fixation has replaced EMG assessment for screw breaches at our institution. There have been no reports on the reliability of EMG when compared with intraoperative imaging.

Materials/Methods: A total of 1006 lumbar screws were placed in 164 consecutive cases. Prospective data was obtained by stimulating all screws and evaluating them with intraoperative $\mathrm{O}$-arm imaging. EMG data was compared with $\mathrm{O}$-arm images to assess pedicle breaches, and all breaches were corrected at the time of initial surgery by palpating the entire trajectory and directly visualizing the pedicle in cases of medial breach.

Results: There were 35 breaches (20 lateral, 15 medial) visualized during surgery with $\mathrm{O}$-arm imaging; $3.5 \%$ of all lumbar screws placed. Of the breaches, 14 screws stimulated below the 12-mA threshold; 9 screws stimulated between 12-20 $\mathrm{mA}$; and 12 breached screws did not generate an EMG response. 40 screws stimulated below a $12-\mathrm{mA}$ threshold but showed no breach on imaging. Using the 12-mA threshold for our analysis, the sensitivity of EMG was $40 \%$, specificity $96 \%$, and a PPV of 26\% (95\% CI: $15-40 \%$ ). All 35 breached screws were corrected during surgery. There were no false-positives in the O-arm group as assessed by direct visualization and palpation of the trajectory. There were no postoperative symptoms caused by breached screws and no patients required re-operation.

Discussion/Conclusion: Our data confirm that the EMG modality for testing breaches is unreliable. In the modern era of intraoperative imaging, lumbar screws should be evaluated for breaches with O-arm rather than relying on EMG stimulation if there are doubts about the position. Direct visualization of the pedicle and palpation of the entire trajectory confirm that $\mathrm{O}$-arm is highly accurate in assessing pedicle breaches.

Paper \#24. Impact of Sarcopenia on Post-Operative Morbidity and Mortality Following Lumbar Spine Surgery

Steven Bokshan, $M D^{l}$, Stephen Marcaccio, $B S^{2}$, Alex Han, $B S^{2}$ J. Mason DePasse, $M D^{1}$, Alan Daniels, $M D^{3}$

${ }^{1}$ Department of Orthopaedics, Warren Alpert Medical School of Brown University, Providence, Rhode Island, ${ }^{2}$ Warren Alpert Medical School of Brown University, Providence, Rhode Island, ${ }^{3}$ Department of Orthopaedics, Division of Spine Surgery- Adult Spinal Deformity Service, Warren Alpert Medical School of Brown University, Providence, Rhode Island

Background/Introduction: Sarcopenia is the loss of muscle mass associated with aging and advanced disease. While sarcopenia has been shown to be an independent predictor of morbidity and mortality in medical and abdominal surgery patients, the impact of sarcopenia on thoracolumbar spine surgery outcomes is unknown.

Materials/Methods: This study retrospectively examined thoracolumbar spine surgery patients over 55 years old $(n=50)$ who underwent surgery from 2003 to 2015 . Each patient's comorbidity burden was determined utilizing the Charlson Comorbidity Index, and the Mirza Surgical Invasiveness Index was utilized to measure procedural complexity. Sarcopenia was diagnosed by measuring total cross sectional area of the psoas muscle (TPA) at the L4 vertebrae using perioperative CT scans. Outcomes measures included postoperative in-hospital complications, length of postoperative inpatient stay, discharge location, and mortality.

Results: Results: Of the 50 patients assessed, 16 were in the lowest tertile for L4 total psoas area (sarcopenic). The sarcopenic patient cohort was significantly older than the non-sarcopenic cohort (mean age of 76.6 vs. $70.8, p=0.027$ ), but did not have a significantly different mean Charlson Comorbidity Index (3.6 vs. 3.2, p $=0.59$ ) or mean Mirza Surgical Invasiveness Index ( 8.8 vs. $6.8, p=0.33$ ). Sarcopenic patients had a hospital length of stay 2.6-fold longer than non-sarcopenic patients (12.3 vs. $4.8, \mathrm{p}=0.005)$, a 4.5 -fold increase in postoperative in-hospital complications ( 1.8 vs. $0.4, \mathrm{p}=0.001)$, and were more likely to require discharge to a rehabilitation or nursing facility $(68.8 \%$ vs $47.1 \%, \mathrm{p}=0.02)$. Sarcopenia conferred an odds ratio of 40.1 for mortality $(\mathrm{p}=0.014)$ with a significantly lower cumulative survival $(\log$ rank $<0.001)$.

Discussion/Conclusion: Sarcopenic patients have a significantly increased risk of in-hospital complications, longer length of stay, increased rates of discharge to rehabilitation facilities, and increased mortality following thoracolumbar spinal surgery. Sarcopenia may be a useful clinical measure of perioperative risk in spine surgery patients.

Paper \#25. Transforaminal Lumbar Interbody Fusion Protects Against Early Pedicle Screw Loosening

David Kim, $M D^{1}$, Riya Joshi, MPH, MBBS ${ }^{2}$, Kevin Baker, Ph.D. ${ }^{3}$, Gyu Ho Lee, BA ${ }^{4}$, Daniel Park, $M D^{5}$, Rick Sasso, $M D^{6}$, Paul Arnold, $M D^{7}$, Jeffrey Fischgrund, $M D^{3}$

${ }^{1}$ New England Baptist Hospital, Boston, MA, 2, Waltham, MA, ${ }^{3}$, Royal Oak, Michigan, ${ }^{4}$, Carlisle, PA, ${ }^{5}$, Royal Oak, MI, ${ }^{6}$, Carmel, IN, ${ }^{7}$, Kansas City, KS

Background/Introduction: Transforaminal lumbar interbody fusion (TLIF) has become a popular surgical option to complement posterolateral fusion (PLF) for treatment of degenerative spinal conditions. Purported advantages of TLIF over PLF alone include enhanced fusion rates, improved sagittal alignment, and direct decompression of the neuroforamen. Earlier studies have examined these issues and yielded inconclusive results. Another often suggested advantage, that TLIF provides superior immediate stability and protects against early pedicle screw loosening, has never been formally examined.

Materials/Methods: A post hoc analysis was performed on an original prospective randomized multicentre trial of 241 patients undergoing single-level instrumented posterior lumbar spinal fusion surgery for degenerative disease. Postoperative computed tomography (CT) and plain radiography were obtained 6 months following surgery and assessed by three independent reviewers. Clinical patient self-reported outcome measures included Visual Analog Scale (VAS) for low back and leg pain, Health-Related Quality of Life Questionnaire Short-Form 36 (SF-36), and Oswestry Disability Index (ODI).

Results: 183 patients had adequate imaging data and were included in the analysis. 89 patients underwent TLIF+PLF (Group A) and 94 patients underwent PLF alone (Group B). At 6-month follow-up, $10.1 \%$ of patients in Group A demonstrated loosening of one or more screws compared to $21.3 \%$ of Group B (ChiSquare value 4.2728; $\mathrm{p}=0.0387$ ). TLIF level or side (right vs. left) was not associated with loosening $(\mathrm{p}=0.1711$ and $\mathrm{p}=0.1402$, respectively) Lucency around the cage was observed in $10.2 \%$ of patients but was not associated with screw loosening $(\mathrm{p}=0.0692)$. 
Although gender was not associated $(\mathrm{p}=0.0833)$, patient age was a significant independent predictor of loosening $(\mathrm{p}=0.0336)$.

Discussion/Conclusion: Early pedicle screw loosening (within 6 months of surgery) occurs at a rate of approximately $16 \%$ per patient and in most cases can only be identified using CT imaging. Associated interbody fusion appears to have a protective effect in reducing rates of early loosening. This protective effect appears independent of actual radiographic evidence of successful interbody fusion and may represent an advantage in weaker bone or for patients in whom early loosening is a particular concern. Increased age is an independent risk factor for early screw loosening.

Paper \#26. Outcomes and Complications of Sacral AlarIliac (S2AI) versus Traditional Iliac Pelvic Fixation

Daniel Kang, $M D^{1}$; Mahati Mokkarala, B.S. ${ }^{2}$, Jacob Buchowski, $M D^{2}$, Panya Luksanapruska, $M D^{3}$, Lawrence Lenke, $M D^{4}$

${ }^{1}$ Madigan Army Medical Center, Tacoma, WA, ${ }^{2}$ Washington University School of Medicine, St. Louis, MO, ${ }^{3}$ Department of Orthopedic Surgery, Faculty of Medicine Siriraj Hospital, Bangkoknoi, Bangkok, ${ }^{4}$, New York, NY

Background/Introduction: Posterior instrumented spinal fusions crossing the lumbosacral junction have a significant rate of complications. There has been increasing popularity of the SacralAlar-Iliac (S2AI) fixation technique; however, the outcomes and complications compared to the well-established iliac screw fixation have not been completely evaluated.

Materials/Methods: We performed a retrospective analysis of adult spinal fusion patients treated at one institution, by two senior surgeons over 10 years (2003-2013) requiring pelvic fixation using either traditional iliac or S2AI screws. Perioperative clinical records were reviewed for demographic data, comorbidities [Charlson Comorbidity Index (CCI)], surgical data, and complications.

Results: Overall there were 421 patients ( $n=270$ iliac screws and $n=151 \mathrm{~S} 2 \mathrm{AI}$ ) included. S2AI compared to Iliac patients were older (59.9 vs 54.4, p<0.001), had a higher mean ASA (2.37 vs 2.26, $\mathrm{p}=0.048)$, higher CCI (2.30 vs $1.95, \mathrm{p}=0.024)$, and were more frequently undergoing a revision procedure $(43.5 \%$ vs $30.0 \%, \mathrm{p}=0.004)$, with no difference in BMI $(27.1$ vs $26.8,0.571)$. We also found the S2AI group compared to Iliac group had a larger number of mean instrumented levels $(11.1 \mathrm{v} 9.0, \mathrm{p}<0.001)$ and more frequently had a PSO procedure $(61.8 \%$ vs $39.8 \%$, $\mathrm{p}<0.001)$. In regards to complications, there was no difference between groups in the rate of screw pullout ( $0 \%$ vs $1.9 \%, \mathrm{p}=0.091)$ and pseudoarthrosis $(1.5 \%$ vs $0 \%, \mathrm{p}=0.109)$. However, the S2AI compared to Iliac group had less broken screws (0\% vs $4.1 \%$, $\mathrm{p}=0.019)$, symptomatic/painful pelvic screw $(2.2 \%$ vs $13.3 \%$, $\mathrm{p}<0.001)$, and fewer reoperations (7.5\% v 20.8\%, p=0.001). The S2AI group had a higher rate of neurologic complications compared to the Iliac group ( $8.2 \%$ vs $0.4 \%, \mathrm{p}=0.257$ ), although the S2AI group underwent more complex, osteotomy procedures.

Discussion/Conclusion: We found the S2AI fixation technique is associated with a lower rate of broken screws, symptomatic/ painful pelvic screws, and fewer reoperations compared to traditional iliac fixation. While the S2AI group had a higher rate of neurologic complications, they were more complex patients requiring osteotomy procedures.
Paper \#27. Reoperation Rates Following Single-Level Discectomy

Patrick Heindel, $B S^{l}$, Alexander Tuchman, $M D^{l}$, Patrick Hsieh, $M D^{l}$, Martin Pham, $M D^{l}$, Neil Patel, $M D^{l}$, Andre Jakoi, $M D^{l}$; Raymond Hah, $\mathrm{MD}^{2}$, John Liu, $M D^{l}$, Zorica Buser, PhD ${ }^{l}$, Jeffrey Wang, $M D^{2}$

${ }^{1}$, Los Angeles, CA, ${ }^{2}$ Keck School of Medicine of USC, Los Angeles, CA

Background/Introduction: Lumbar discectomy is the most commonly performed procedure for treatment of radiculopathy due to lumbar disc herniation. Randomized clinical trials have demonstrated the advantage of surgical removal of herniated disc material over non-surgical treatment options, allowing for a more rapid reduction in symptoms and return of function. Population-level data regarding reoperation following single level hemilaminotomy and discectomy is limited.

Materials/Methods: Data were collected for patients undergoing single-level lumbar discectomy between January 2007 and September 2014 using the commercially available PearlDiver database (PearlDiver, Inc., Fort Wayne, IN, USA). The nationwide Humana private insurance database was queried for patients billed with the Current Procedural Terminology (CPT) code for our index procedure, hemilaminotomy and removal of disc material. Patients receiving concurrent lumbar surgeries were excluded from the index group. The index group was then followed for 3 months, 6 months, 1 year, 2 years, and 4 years for recurrent lumbar surgery, including spinal fusion, laminectomy, and additional discectomy.

Results: Analysis of data obtained from 13,654 patient records revealed a rate of additional lumbar surgeries following singlelevel discectomy of $3.95 \%(539 / 13654)$ within 3 months and $12.2 \%(766 / 6274)$ within 4 years from the index procedure. Lumbar spinal fusion was performed on $5.9 \%(370 / 6274)$ of patients within 4 years, with interbody fusion in $75.4 \%(279 / 370)$ and multi-level fusion in $47.0 \%(174 / 370)$ of cases. Revision discectomy with no subsequent surgeries occurred in $2.71 \%$ (170/6274) of patients followed out to 4 years. Patients who received a revision discectomy within 2 years of the index procedure went on to receive lumbar fusion at a rate of $38.4 \%$ (48/125) within the 4 years following their second procedure.

Discussion/Conclusion: In this study, we report a reoperation rate of $12.2 \%$ after single-level discectomy. We show that the rate of progression to lumbar fusion after revision discectomy is $38.4 \%$ within 4 years of reoperation. To our knowledge, this is the largest population study delineating reoperation rates across the US after single-level lumbar discectomy. Further studies are needed regarding the best treatment algorithm in patients with re-herniation or iatrogenic instability following lumbar discectomy.

Paper \#28. The Effect of an Adjustable Hinged Operating Table on Lumbar Lordosis during Lumbar Surgery

Arjun Sebastian, $M D^{l}$, Amin Mohamed Ahmed, $M D^{2}$, Ilyas Aleem, MD, MSc, FRCSC ${ }^{2}$; Bradford Currier, M.D. ${ }^{1}$, Michelle Clarke, $M D^{l}$, Paul Anderson, $M D^{3}$; Ahmad Nassr, $M D^{2}$;

${ }^{1}$, Rochester, MN, ${ }^{2}$ Mayo Clinic, Rochester, $\mathrm{MN},{ }^{3}$ University of Wisconsin, Madison, WI

Background/Introduction: Hinged operative tables have the theoretical advantage of allowing surgeons to adjust lumbar spine positioning to facilitate both decompressive and fusion surgery. The amount of lumbar lordosis in neutral, flexion, and extension positions has not been quantified prospectively using a hinged table for prone lumbar spine surgery. 
Materials/Methods: Thirty patients scheduled to undergo elective lumbar decompression and/or discectomy at a single institution were enrolled. Preoperative MRI as well as standing $\mathrm{x}$-rays taken in neutral, maximal flexion, and maximal extension were obtained. Following prone positioning on an OSI Axis Jackson table, x-rays in neutral, maximal flexion, and maximal extension were taken. Total lumbar lordosis was calculated in all 6 images utilizing the Cobb method by two physicians. The degeneration of the lumbar intervertebral discs was graded using Pfirrmann MRI disc grades. Rater reliability was calculated, and comparisons of lumbar lordosis between radiographs were made.

Results: The average age of the cohort was 42.5 years. Interrater and intra-rater reliability was strong with intraclass coefficient ranges of 0.78-0.89 and 0.77-0.82 respectively. Lumbar lordosis on the operative table was $56.5^{\circ} \pm 2.1^{\circ}, 43.6^{\circ} \pm 2.2^{\circ}, 63.2^{\circ} \pm 2.0^{\circ}$ in neutral, flexion, and extension respectively compared to $46.9^{\circ}$ $\pm 3.1^{\circ}, 33.2^{\circ} \pm 2.8^{\circ}, 52.3^{\circ} \pm 3.3^{\circ}$ on the standing films. Average flexion $\left(12.9^{\circ} \pm 1.1^{\circ}\right)$ and extension $\left(6.7^{\circ} \pm 1.2^{\circ}\right)$ were significantly different from neutral on the operative table $(\mathrm{p}<.001)$. Lordosis was significantly higher on the operative table $(\mathrm{p}<.001)$ in comparison to standing films. Total range of motion (maximal extension to maximal flexion) was $19.6^{\circ} \pm 1.9^{\circ}$ on the table and $19.1^{\circ} \pm 2.0^{\circ}$ on standing films without significant difference $(\mathrm{p}$ $=0.42$ ). Average Pfirrmann disc grade for the cohort was $2.77 \pm$ 0.10 . No significant correlation was found between disc score and total lumbar range of motion $(\mathrm{p}=0.40)$.

Discussion/Conclusion: A hinged operative table allowed for a physiologic arc of motion of nearly $20^{\circ}$ from flexion to extension. Measurements of lumbar flexibility were unaffected by disc degeneration. A considerable amount of lumbar sagittal motion can be obtained on hinged operative tables without decreasing overall lumbar lordosis below physiologic levels.

\section{Paper \#29}

Total en bloc Spondylectomy for Locally Aggressive and Primary Malignant Tumors of The Lumbar Spine: Surgical and Oncological Outcomes

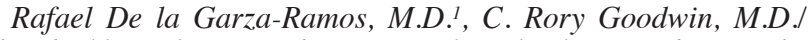
Ph.D. ${ }^{l}$, Ali Bydon, M.D. ${ }^{1}$, Jean-Paul Wolinsky, M.D. ${ }^{1}$, Timothy Witham, MD ${ }^{2}$, Ziya Gokaslan, M.D. ${ }^{3}$; Daniel Sciubba, M.D. ${ }^{.}$

${ }^{1} J o h n s$ Hopkins Hospital, Baltimore, Maryland, 'Johns Hopkins University School of Medicine, Baltimore, MD, ${ }^{3}$ Brown University, Providence, Rhode Island

Background/Introduction: Total en bloc spondylectomy (TES) in the lumbar spine is relatively uncommon, given that most spinal tumors present in the thoracic region and the surgical challenge associated with the unique anatomy of the lumbar spine - proximity of lumbar plexus, iliopsoas muscle, major vessels, and bowel.

Materials/Methods: A retrospective review of neurosurgical patients undergoing lumbar TES for primary aggressive/ malignant tumors at a single institution between 2004 and 2014 was performed. Patient characteristics, tumor characteristics (primary pathology, Enneking stage, and Tomita Type), and surgical data were ascertained from clinical notes. Outcomes included perioperative complication rates, reoperation rates for instrumentation failure/pseudoarthrosis, disease-free survival, and overall survival.

Results: A total of 21 patients were identified and followed for an average of 51 months (range $6-117$ months). The most common primary histology was chordoma in $52.4 \%$ of cases, followed by sarcoma in $19 \%$. The perioperative complication rate was $61.9 \%$, and the most common complication was wound infection in $23.8 \%$ of cases. Eight patients $(38.1 \%)$ required reoperation for instrumentation failure/pseudoarthrosis on average 26 months after index spondylectomy; this rate was not significantly different for single vs. multi-level spondylectomy $(\mathrm{p}=0.346)$. The 5 -year disease-free survival was $66.5 \%$ and the 5 -year overall survival was $82 \%$. There were no significant differences in disease-free survival and overall survival based on tumor pathology, Enneking stage, or Tomita Type by the log rank tests.

Discussion/Conclusion: Although the perioperative complication rate and instrumentation failure rate after total en bloc spondylectomy is considerably high, this technique may provide a high disease-free and overall survival rate for patients with primary malignant and locally aggressive tumors of the lumbar spine.

Paper \#30. Randomized Controlled Trial of Minimally Invasive Sacroiliac Joint Fusion Using Triangular Titanium Implants vs. Non-Surgical Management for Sacroiliac Joint Dysfunction

David Polly, Jr, $\mathrm{MD}^{l}$; Jonathan Sembrano, $\mathrm{MD}^{2}$, Charles Ledonio, $M D^{3}$, Daniel Cher, $M D^{4}$

${ }^{1}$ University of Minnesota, Minneapolis, MN, ${ }^{2}$, Minneapolis , MN, ${ }^{3}$, Minneapolis, MN, ${ }^{4} \mathrm{SI}$ - Bone, Inc., San Jose, CA

Background/Introduction: Sacroiliac (SI) joint dysfunction is a prevalent cause of unremitting low back pain. Does minimally invasive SIJ fusion provide superior outcomes compared to nonsurgical treatment?

Materials/Methods: 148 subjects with SI joint dysfunction were randomly assigned at 19 US centers to minimally invasive SI joint fusion with triangular titanium implants $(\mathrm{N}=102)$ or nonsurgical management (NSM, n=46). Pain, disability and quality of life scores were collected at baseline and at 1,3,6 and 12 months. Success rates were compared using Bayesian methods. Crossover from nonsurgical to surgical care was allowed after the 6month study visit was complete.

Results: Six-month success rates were higher in the surgical group $(81.4 \%$ vs. $26.1 \%$, posterior probability of superiority $>0.9999$ ). Clinically important ( $\geq 15$ point) ODI improvement at 6 months occurred in $73.3 \%$ of the SIJ fusion group vs. $13.6 \%$ of the NSM group $(\mathrm{p}<.0001)$. Improvements in SIJ pain and ODI were sustained at 12 months in the surgical group. Subjects who crossed over had improvements in pain, disability and quality of life similar to those in the original surgical group. Adverse events were slightly more common in the surgical group (1.3 vs. 1.1 events per subject, $\mathrm{p}=0.3063$ ). One patient underwent immediate revision surgery for neuropathic pain related to implant malposition. 77 and $78 \%$ of subjects assigned to SIJ fusion were "very satisfied" with SIJ fusion at 6 and 12 months, respectively.

Discussion/Conclusion: In this study, minimally invasive SI joint fusion using triangular titanium implants was more effective than nonsurgical management at one year in relieving pain, improving function and improving quality of life in patients with SI joint dysfunction.

Paper\#31.SpinalStenosisin the Absence of Spondylolisthesis: Can Interlaminar Stabilization at Single and Multi-levels Provide Sustainable Relief?

Byung Jo Yoon, M.S. ${ }^{1}$, Daniel Shein, $M D^{2}$, Samuel Grinberg, $B A^{2}$, Frank Cammisa, $M D^{2}$, Celeste Abjornson, $P h D^{2}$

${ }^{1}$ Hospital for Special Surgery, New York, NY, ${ }^{2}$ Hospital for Special Surgery, New York, New York

Background/Introduction: In the absence of instability or 
deformity (non-spondylolisthesis), fusion procedures are generally not recommended. However, decompression alone often does not provide long-term clinical success of intractable leg and back pain. Decompression with Interlaminar stabilization (ILS) offers a new clinical continuum of care by providing a non-fusion surgical alternative. The objective of this study is to examine the sustainability of decompression with ILS and to further understand the clinical success at either a single level or two levels as a surgical treatment for non-spondylolisthesis patients with spinal stenosis.

Materials/Methods: Under FDA-regulated IDE study, a total of 322 patients from 21 sites in the US were enrolled between 2006 and 2010 in the prospective, randomized trial. This investigation focuses only on the subset of patients (116 total) who were treated with decompression + ILS (coflex ${ }^{\circledR}$, Paradigm Spine, NY, NY) at 1 or 2 levels and who did not present with spondylolisthesis preoperatively. The patients were assessed before and after surgery at 6 weeks and $3,6,12,18,24$, and 48 months with patient self-assessment, physical and neurological examination, and radiographic evaluation.

Results: ODI scores difference at all follow-up time points were statistically significant compared to baseline out to 48 months for both 1 and 2 level patients, but were not different at any time point between patient populations. At 48 months follow-up, there was no statistically significant difference in ODI $\geq 15$ point improvement between patient populations. For both treatments, over $90 \%$ of patients reported no persistent new or increasing sensory or motor deficit $(\mathrm{p}=0.238)$. At 48 months, $97.3 \%$ of 1 -level and $97.1 \%$ of 2-level reported $\geq 20 \mathrm{~mm}$ improvement in VAS leg pain. Patients reported improvement in their physical state according to SF-12 scores (90.6\% of 1-level, $100 \%$ of 2-level, $\mathrm{p}=0.238$ ). Patient satisfaction at 48 months was high (97.2\% of 1-level, $100 \%$ of 2-level).

Discussion/Conclusion: The therapeutic sustainability for the treatment of spinal stenosis without spondylolisthesis with interlaminar stabilization at 1 or 2 levels in the lumbar region has been shown to be safe and efficacious for patients who have failed conservative treatment.

Paper \#32. Outcomes of Lumbopelvic Fixation in the Treatment of Complex Sacral Fractures Using Minimally Invasive Surgical Techniques

Ehsan Jazini, $M D^{l}$, Emeka Nwodim, $M D^{2}$, Oliver Tannous, $M D^{l}$, Nicholas Caffes, $B S^{l}$, Timothy Costales, $B S^{l}$; Tristan Weir. $\underline{B S}^{l}$, Eugene Koh, $M D, P h D^{l}$, Kelley Banagan, $M D^{l}$, Daniel Gelb, $M D^{l}$, Steven Ludwig, $M D^{l}$

\section{1, Baltimore, MD, ${ }^{2}$, Baltimore , MD}

Background/Introduction: This is a retrospective cohort study of minimally invasive lumbopelvic fixation (LPF) as a treatment for complex sacral fractures at a single institution. Complex sacral fractures with vertical and anterior pelvic ring instability treated with traditional fixation methods are associated with high rates of failure and poor clinical outcomes. Supplemental LPF has been applied for additional stability to help with fracture union. The purpose of this study was to determine whether minimally invasive LPF provides reliable fracture stability and acceptable complication rates in cases of complex sacral fractures.

Materials/Methods: Twenty-eight patients who had undergone LPF at our level I trauma center from 2008 through 2014 were retrospectively selected from a pool of 105. Patients underwent posterior sacroiliac fixation, with or without anterior fixation, followed by minimally invasive LPF. Main outcomes were return to the operating room (OR) for instrumentation revision secondary to loss of correction or failure of fixation, return to the OR for treatment of infection, and radiographic evaluation to assess for loss of reduction. Injury Severity Score (ISS), transfusion requirements, length of hospital stay, postoperative day at mobilization, and mortality were also evaluated.

Results: Three (11\%) patients returned to the OR for infection. One $(3.5 \%)$ required revision surgery for instrumentation malposition and neurological deficit. No patient required return to the OR for revision surgery because of failure of instrumentation or loss of correction. Average length of surgery was 3.9 hours. Estimated average blood loss was $192 \mathrm{~mL}$, and average transfusion requirement was 2.2 units of packed red blood cells. The majority of patients were mobilized on postoperative day 4 . No mortalities occurred.

Discussion/Conclusion: This is currently the largest reported series of high-energy complex sacral fractures treated with minimally invasive LPF. Our series reinforces the principle that appropriate reduction and adequate biomechanical stability can be achieved in high-energy pelvic and sacral fractures with acceptable complication rates. Although other studies have evaluated biomechanical stiffness and complication rates among cases treated with open LPF, none have specifically elucidated the role of minimally invasive LPF.

Paper \#33. Evaluation of Sacral End-plate Penetrating Screw for Lumbosacral Fusion: A Cadaveric Biomechanical Study

Grigor Grigoryan, $\mathrm{MD}^{l}$, Wayne Cheng, $\mathrm{MD}^{2}$, Serkan Inceoglu, PhD ${ }^{3}$, Olumide Danisa, $M D^{4}$, Yusuf Akpolat, $M D^{5}$, Phillip Grigsby, $B S^{5}$

${ }^{1}$ Loma Linda University, Loma Linda, california, ${ }^{2}$, Colton, CA, ${ }^{3}$ Loma Linda University, Department of Orthopedic Surgery, Loma Linda, CA, ${ }^{4}$ Loma Linda University, Department of Orthopaedic Surgery, Loma Linda, CA, ${ }^{5}$, Loma Linda, CA

Background/Introduction: Cortical bone trajectory(CBT) technique has been shown to provide equivalent and possibly superior purchase in osteoporotic bone. CBT employs a more medial starting point. Currently, the accepted method of instrumenting to the sacrum for lumbosacral fusion using CBT is the S1-Ala screw (S1AS). A novel endplate penetrating screw (EPS) trajectory may provide a more optimal screw purchase; however, to our knowledge no biomechanical cadaveric studies have been performed to investigate the pull-out strength of this trajectory to the standard S1AS.

Materials/Methods: This is a cadaveric biomechanical study. Eight fresh frozen human cadaveric sacra were used, four of which had normal bone mineral density (BMD) $(1.038 \pm 0.105 \mathrm{~g} /$ $\mathrm{cm} 2)$, and the other four had low BMD $(0.689 \pm 0.100 \mathrm{~g} / \mathrm{cm} 2)$. Each side of the sacra were randomly assigned to either EPS or S1AS trajectory using $7.5 \mathrm{~mm}$ diameter screws ranging $40 \mathrm{~mm}$ to $50 \mathrm{~mm}$ in length using fluoroscopy. Reflective markers were used to quantify relative motion using a motion analysis system. $6 \mathrm{~mm}$ of relative motion was designated as an endpoint to cyclic loading. Each screw underwent cyclic loading of $4 \mathrm{Nm}$ at $2 \mathrm{~Hz}$ for 20000 cycles unless they reached the designated endpoint sooner. The screws were then pulled out vertically at a rate of $5 \mathrm{~mm} / \mathrm{min}$ and the pull-out force was recorded.

Results: The mean pull-out force following cyclic loading was significantly higher $(\mathrm{P}<0.0001)$ for fixation with EPS $(1712 \mathrm{~N} \pm 886 \mathrm{~N})$, than fixation with $\mathrm{S} 1 \mathrm{AS}(641 \mathrm{~N} \pm 725 \mathrm{~N})$. The difference was more pronounced in osteoporotic bone with the EPS $(1075 \mathrm{~N})$ having on average 2.95 times higher pull-out force compared to S1AS (mean365N). In sacra with normal BMD the pull-out force was 2.56 times higher for EPS(mean 2349N).

Discussion/Conclusion: The EPS trajectory appears to have 
much higher pullout force compared to the S1AS which is the current preferred method of instrumentation when using CBT trajectory. Amongst all the subgroups, the screws that had the lowest pull-out force were the S1AS in osteoporotic bone. This raises the concern for potential hardware failure risk. EPS trajectory is a viable and far superior option for instrumentation.

Paper \#34. Does Single- versus Two-Stage Pedicle Subtraction Osteotomy in Spinal Deformity Surgery Influence Perioperative Complications?

Daniel Kang, $M D^{l}$, Ronald Lehman, $M D^{2}$, Lawrence Lenke, $M D^{3}$, Panya Luksanapruska, $M D^{4}$, Todd Chapman, $M D^{5}$, Lionel Metz, $M D^{5}$, Jamal McClendon, $J r$., $M D^{5}$

${ }^{1}$ Madigan Army Medical Center, Tacoma, WA, ${ }^{2}$, New York, NY, ${ }^{3}$, New York, NY, ${ }^{4}$ Department of Orthopedic Surgery, Faculty of Medicine Siriraj Hospital, Bangkoknoi, Bangkok, ${ }^{5}$ Washington University School of Medicine, St. Louis, MO

Background/Introduction: PSO for complex spinal deformity can be challenging with a high rate of perioperative complications. We evaluated the rate of perioperative complications comparing single- versus two-stage PSO.

Materials/Methods: Adult spinal deformity patients undergoing all-posterior PSO were retrospectively analyzed as two cohorts, single-stage (SS) vs. two- staged (TS). Perioperative clinical records were reviewed, and any complications occurring within the first two months postoperatively were recorded.

Results: 173 consecutive adult patients were identified that underwent PSO, with 103 patients meeting inclusion criteria and having complete data (59 SS and $44 \mathrm{TS}$ ). There were no differences in demographics (age, BMI, gender, ASA classification, Charlson Comorbid Index (CCI), smoking status, primary $\mathrm{v}$ revision surgery, preop diagnosis). We found no difference in total \# of complications [SS 21 (35.6\%) vs TS $13(29.5 \%), \mathrm{p}=0.519]$, or number of neurologic deficits (SS 7 v TS $11, \mathrm{p}=0.082$ ). We found a difference in total EBL (SS 2632 vs TS $3246 \mathrm{~mL}, \mathrm{p}=0.019$ ), and total \# of hospital days (SS 9.42 vs TS 14.3 days, $\mathrm{p}=0.00$ ). However, there were no statistical differences in total surgery time (SS 617 vs TS 728 min, p=0.129), total \# of intraoperative pRBC transfusions (SS $4.66 \mathrm{v}$ TS 5.25 units, $\mathrm{p}=0.332$ ), or total \# of postoperative $\mathrm{pRBC}$ transfusions (SS $1.47 \mathrm{v}$ TS $1.86, \mathrm{p}=0.391$ ).

Discussion/Conclusion: There were no differences in perioperative complications between single- versus two-stage PSO procedures for adult spinal deformity patients. Two-staged PSO resulted in greater EBL and increased length of hospitalization, but there were no differences in total surgery time or \# of intraop/ postop blood transfusions.

Paper \#35. Surgical Timing, and not Surgical Invasiveness, is Predictive of Perioperative Outcomes After Long Posterior Fusions for Adult Spinal Deformity: A Need for Speed?

Andre Samuel, $B B A^{1}$, Michael Fu, $M D^{2}$, Nidharshan Anandasivam, $B S^{l}$, Adam Lukasiewicz, $M S c^{l}$, Matthew Webb, $A B^{l}$, Han Jo Kim, $M D^{2}$, Jonathan Grauer, $M D^{3}$

${ }^{1}$ Yale School of Medicine, New Haven, CT, ${ }^{2}$ Hospital for Special Surgery, New York, NY, ${ }^{3}$, New Haven, CT

Background/Introduction: Morbidity is high after long posterior fusions for adult spinal deformity. While previous reports have demonstrated an association between perioperative outcomes and the extent of spinal fusion with modern surgical techniques that minimize the risk of acute intraoperative errors, operative time (and the associated effects of longer anesthesia time and bleeding) may be the leading driver of postoperative complications.

Materials/Methods: All patients, age 40 or older, undergoing posterior spinal fusion for spinal deformity were identified in the 2010 - 2013 National Surgical Quality Improvement Program (NSQIP) database. Multivariate analysis was used to determine the independent effects of operative timing, surgical invasiveness (number of levels fused, combined anterior-posterior fusions, pelvic fixation, osteotomies, perioperative transfusion), and other patient factors on perioperative outcomes.

Results: A total of 1,066 patients undergoing posterior spinal fusion for adult spinal deformity were identified. The overall rate of complications was $16.23 \%$, while the rate of serious complications was $6.10 \%$. In multivariate analysis age 50 years and older (odds ratio [95\% C.I.]: 2.91 [1.32 - 6.41] for ages 50 59), ASA class of IV and above (4.07 [1.96 - 8.44]), and operative timing of 420 minutes and longer (2.43 [1.07 - 5.52] for 420 - 479 minutes; Figure 1) were predictive of any complication while other operative factors (number of levels fused, anterior fusion, osteotomies, pelvic fixation, and perioperative transfusion) were not statistically significant risk factors. Age 80 years and older (7.39 [1.79 - 30.50]), ASA class of IV and above (3.37 [1.18 - 9.63]), and operative timing of 480 minutes and longer (9.46 [1.79 - 49.95] for 480 - 549 minutes) were predictive of serious complications while other operative factors were not.

Discussion/Conclusion: Operative time is a better predictor of perioperative complications than other operative factors. Rather than avoidance of more invasive surgical procedures, such as longer posterior fusions, combined anterior and posterior fusions, and pelvic fixation, which have been shown to improve stability and long-term outcomes, it may be that emphasis should be placed on reduction of overall operative time while still performing adequate deformity correction.

Paper \#36. Low Fusion Grade Does Not Impact Two-Year Health Related Quality of Life Unless Rod Fracture Occurs for Adult Spinal Deformity

Farbod Rastegar, $M D^{l}$, Tamir Ailon, $M D^{2}$, D. Hamilton, $M D^{3}$, Robert Hart, M.D. ${ }^{4}$, Eric Klineberg, $M D^{5}$, Virginie Lafage, $P h D^{6}$, Shay Bess, $M D^{7}$, Alan Daniels, $M D^{8}$, Justin Smith, $M D^{9}$, Christopher Shaffrey, $M D^{10}$

${ }^{1}$ Oregon Health \& Science University, Portland, Oregon, ${ }^{2}$ Vancouver Spine Surgery Institute, Vancouver, BC, ${ }^{3}$ University of Pittsburgh Medical Center, Pittsburgh, PA, ${ }^{4}$ Oregon Health and Science University, Portland, OR, ${ }^{5}$ University of California Davis, Sacramento, California, ${ }^{6}$ Hospital for Special Surgery, New York, NY, ${ }^{7}$ NYU Hospital for Joint Diseases, White Plains, NY, ${ }^{8}$ Department of Orthopaedics, Division of Spine SurgeryAdult Spinal Deformity Service, Warren Alpert Medical School of Brown University, Providence, Rhode Island, 'University of Virginia, Charlottesville, VA, ${ }^{10}$ University of Virginia Medical Center, Charlottesville, VA

Background/Introduction: Pseudarthrosis after adult spinal deformity (ASD) surgery may result in instrumentation failure and need for revision. However, the correlation between fusion grade with or without rod fractures and HRQoL is not known. We assessed the association between HRQoL, fusion grade, and rod fracture with and without revision surgery.

Materials/Methods: A prospective, multi-center ASD database with 2 year followup data was reviewed. Fusion grade was determined on plain films using published criteria; Grade III or IV at one or more segments was considered failure of fusion. HRQoL measures included ODI, SF-36 PCS and MCS, and SRS-22r 
Total. Radiographs from 205 patients with posterior or combined anterior/posterior thoracolumbar fusion were assessed.

Results: Radiographic evidence of solid fusion was achieved in $73 \%$ of patients. HRQoL were similar between those that achieved versus did not achieve fusion (ODI 27.3/26.2; PCS 40.6/40.8; MCS 49.4/54.2; SRS-Total 3.7/3.8; SRSS 4.5/4.5) ( $>>0.05)$. Rod fractures occurred in $35(17 \%)$ of patients at an average of 32.7 months postoperatively. Of these 35 patients, 17 required revision and 18 did not. Comparing patients with no rod fractures to those with rod fractures and those with rod fractures requiring revision, we found a progressive and significant worsening in ODI $(\mathrm{p}<0.0001), \operatorname{PCS}(\mathrm{p}=0.02)$, MCS $(\mathrm{p}=0.03)$, and SRS-Total $(\mathrm{p}=0.006)($ Table 1$)$.

Discussion/Conclusion: Failure of fusion at $\geq 1$ level after ASD surgery did not significantly impact 2-year HRQoL. The occurrence of rod fractures requiring revision surgery was associated with significantly worse 2-year HRQoL outcomes as compared to no rod fractures. Fusion grade was less sensitive to clinical outcomes than the occurrence of rod fracture, especially for patients requiring revision surgery.

Paper \#37. The Impact of Surgical and Patient Factors on Lumbar Lordosis for Single-Level Lumbar Fusion

Grace Xiong, $B A^{1}$, Serena $H u, M D^{l}$, Todd Alamin, $M D^{l}$, Ivan Cheng, $M D^{2}$

${ }^{1}$, Redwood City, CA, ${ }^{2}$ Stanford University Medical Center, Redwood City, CA

Background/Introduction: Greater investigation of the impact of surgical factors on spinal balance and patient-reported outcomes is needed to provide surgeons with adequate information to assess how their practice might affect a patient's overall alignment and post-surgical outlook. In particular, the diversification of approaches, while showing significant differences in operative time and blood loss in some studies, has little data examining the impact of these approaches on overall spinal balance. The purpose of this study was to examine the effect of patient and surgical factors on lumbar lordosis in the setting of single-level fusions.

Materials/Methods: Consecutive patients who received a single-level lumbar fusion performed at a single institution between 2012 and 2014 were identified by CPT code or operative report documentation. Basic demographic data, operative details, and pre- and post-operative notes were examined. Pre-operative and the most recent post-operative radiograph were measured for lumbar lordosis (LL) and interbody lordosis. VAS-Back, VAS-Leg, and Oswestry Disability Index (ODI) scores were also collected from chart review.

Results: 80 patients were identified meeting criteria with an average followup of 436 days. The degree of LL correction was significantly different based on level fused ( $\mathrm{p}=0.0018)$, with the greatest lordosis correction at the L4-L5 level. Age, sex, BMI, smoking status, and Charlson co-morbidities did not have a significant effect on LL. ALIF cases had greater interbody lordosis correction compared with XLIF or TLIF approaches, however this significance was diminished when controlling for fused level. Other surgical factors such as the use of a percutaneous pedicle screw system versus an open technique did not have a significant effect. ODI scores decreases after surgery were significantly correlated with the degree of lordosis correction $(\mathrm{p}=0.0003)$.

Discussion/Conclusion: This retrospective study found significant correlations between the degree of lumbar lordosis correction and decrease in ODI scores following single-level lumbar fusion. No significant correlation was found between patient factors and lordosis correction, and more study is needed to elucidate the relationship between surgical approaches and lordosis correction. Examination across larger datasets will also determine which patterns are consistent and widely applicable.

Paper \#38. Sagittal Imbalance in Degenerative Scoliosis Can Be Corrected By Addressing Only the Symptomatic Levels

Philip Louie, $M D^{I}$, Steven Presciutti, $M D^{2}$, Michael Hellman, $M D^{l}$, Christopher Dewald, $M D^{l}$, Howard An, $M D^{l}$

${ }^{1}$ Rush University Medical Center, Chicago, IL, ${ }^{2}$ Emory University, Atlanta, GA

Background/Introduction: The optimal method of addressing the loss of lumbar lordosis (LL) often present in degenerative scoliosis (DS) is controversial. The goal is to achieve an adequate decompression as well as correct the sagittal and coronal deformities. An important distinction is whether or not the loss of LL often seen is truly structural or more positional in nature. The purpose of this study is to determine (1) if the loss of LL often associated with DS is structural or rather largely due to positional factors secondary to spinal stenosis, and (2) if the entire DS curve or a lesser local fusion of only the neurologically symptomatic levels need to be addressed.

Materials/Methods: Pre- and post-operative radiographs and preoperative MRIs were analyzed for LL, pelvic incidence (PI), and pelvic tilt (PT). Patients were included if they had DS, a loss of LL preoperatively $\left(\mathrm{PI}-\mathrm{LL}>9^{\circ}\right)$, and underwent a decompression and instrumented fusion of only their symptomatic levels.

Results: Sixty-eight consecutive patients with average followup of 22 months were reviewed. Preoperative MRIs and lumbar extension radiographs revealed an average $\mathrm{LL}$ of $42^{\circ}\left(10-66^{\circ}\right)$ and $48^{\circ}\left(20-74^{\circ}\right)$, respectively. LL postoperatively was corrected to a mean of $44^{\circ}\left(14-68^{\circ}, \mathrm{p}<0.001\right)$, resulting in an average PI-LL of $9^{\circ}$. Of patients who achieved PI-LL within $9^{\circ}$ on their extension radiographs, $63 \%$ of patients were within $9^{\circ}$ on their postoperative films. Only $15 \%$ of patients were not able to achieve that mismatch on extension radiographs ( $\mathrm{p}=0.001, \mathrm{OR}=9.58)$. Similarly, $54 \%$ of patients were able to achieve a PI-LL $<9^{\circ}$ postoperatively (as on pre-operative MRI) In contrast, only $22 \%$ of patients achieved that goal postoperatively if their mismatch was greater than $9^{\circ}$ on their MRI ( $\mathrm{p}=0.003, \mathrm{OR}=4.25)$.

Discussion/Conclusion: Despite an average PI-LL $>20^{\circ}$ preoperatively, LL was corrected to within $9^{\circ}$ of the PI in this cohort of symptomatic DS patients with decompression and instrumented fusion of their symptomatic levels only, without exposing them to the complications associated with longer instrumented fusions and/or osteotomies. Importantly, the loss of LL is positional rather than structural and the amount of LL correction achievable can be predicted from preoperative MRI and extension radiographs.

Paper \#39. A New Classification and Treatment Algorithm for Acute and Subacute Adolescent Spondylolysis

Jeffrey Dick, $M D$

Twin Cities Orthopedics, Edina, MN

Background/Introduction: Stress fractures of the pars interarticularis seem to be more prevalent likely due to trends toward overtraining in adolescent sports. Most providers make no attempt to get the fracture to heal and many of these patients have ongoing pain of varying degrees into adulthood and seek out numerous treatments and sometimes surgery. There could 
be substantial cost savings and improved quality of life in these patients if the acute stress fractures could heal.

Materials/Methods: Patients were studied prospectively. Fractures were classified based on their MRI and CT appearance into the following groups: TYPE I: High signal on STIR images but no fracture visible on CT. TYPE II: High signal on STIR images and a partial crack visible at least on one side on CT. TYPE III: High signal on STIR images with a complete crack at least on one side on CT. TYPE IV: Normal signal on STIR images and a complete crack on CT. Type IV fractures were excluded. All patients were treated with Vitamin D3 and calcium supplements. Type I and Type II fractures were told to abstain from athletic activity for three months. TYPE III Fractures wore a TLSO \#5 brace molded in neutral lordosis for a period of 6 months. CT scans were repeated post treatment.

Results: 53 patients were treated with Type I, II or III fractures. $100 \%$ of the TYPE I fractures healed. $93 \%$ of the Type II fractures healed. 55\% of the Type III fractures healed.

Discussion/Conclusion: This shows that virtually all Type I and II fractures heal when treated appropriately and in a timely fashion. Adolescent athletes should be evaluated with MRI if they have ongoing symptoms of low back pain that last longer than two or three weeks. With proper immobilization and nutritional treatment the majority of these patients can be spared from a lifetime of recurring pain and healthcare resources can be redirected.

Paper \#40. Downstream Mechanisms of Aryl Hydrocarbon Receptor Activation: Inhibitory Effects on Osteogenic Differentiation

Danielle Chun, $B A^{1}$, Chawon Yun, $P h D^{2}$, Joseph Weiner, $B S^{3}$, Ralph Cook, $B S^{1}$; Michael Schallmo, BS ${ }^{3}$, Ryan Freshman, $B S^{1}$, Jonghwa Yun, N/A ${ }^{4}$, Anjan Ghosh, N/A4, Wellington Hsu, M.D. ${ }^{5}$, Erin $\mathrm{Hsu}, \mathrm{PhD}^{3}$

${ }^{1}$ Northwestern University Department of Orthopaedic Surgery, Chicago, IL, ${ }^{2}$ Northwestern University Department of Orthopaedic Surgery , Chicago, IL, ${ }^{3}$ Northwestern University Department of Orthopaedic Surgery, Chicago , IL, ${ }^{4}$, Chicago, IL, ${ }^{5}$ Northwestern Memorial Hospital, Chicago, Illinois

Background/Introduction: Cigarette smoking is associated with increased rates of pseudarthrosis after spine fusion procedures. Numerous toxic ligands for the Aryl hydrocarbon receptor are present in cigarette smoke, and recent evidence suggests that Ahr activation may inhibit osteogenic differentiation. We showed previously that activation of the Aryl hydrocarbon receptor by dioxin inhibits bone regeneration and spine fusion in the rat. The purpose of this study was to elucidate downstream mechanisms of dioxin action, and to identify therapeutics that mitigate the effects of dioxin on bone.

Materials/Methods: Bone marrow stromal cells were isolated from rat femurs and tibiae and cultured under standard or osteogenic conditions. Factors that are critical to osteogenesis were evaluated after exposure to vehicle control, dioxin, Ahr antagonists, or antagonists + dioxin. These included markers for osteogenic differentiation (ALP activity, cell migration rate, and mineralization capacity) and RNA and protein expression for several pro-osteogenic genes. Antagonists evaluated were: alphanaphthoflavone (ANF), resveratrol (Res), 3,3'-diindolylmethane (DIM), and luteolin (Lut).

Results: We found that dioxin inhibits ALP activity, BMSC migratory capacity, and matrix mineralization. Co-treatment with each of the 4 antagonists mitigates these effects. Dioxin was also found to inhibit BMSC chemotaxis towards various chemoattractants. Co-treatment with ANF and Res generally rescued the anti-chemotactic effects of dioxin to a greater degree than DIM and Lut. RNA and protein expression of numerous pro-osteogenic genes was down-regulated by dioxin exposure. Co-treatment of BMSC with Ahr antagonists prevented dioxininduced expression changes of Col2, Col12, Phex, MMP-3, and MMP-13 proteins to varying degrees.

Discussion/Conclusion: Ahr activation may play a critical role in the adverse effects of cigarette smoke on bone healing. Three of the Ahr antagonists used in this study are naturallyoccurring phytochemicals, some of which are under investigation for chemoprotective effects, and are available over-the-counter as food supplements. The identification of safe Ahr antagonists that provide a protective effect against the negative impacts of dioxin on bone could answer the call for a therapeutic that may protect against the deleterious effects of smoking on bone healing.

Paper \#41. Peptide Amphiphile-Collagen Slurry Nanogel Composite as an Improved BMP-2 Carrier for Spinal Arthrodesis

Ralph Cook, $B S^{1}$, Joseph Weiner, $B S^{2}$, Danielle Chun, $B A^{1}$, Sungsoo Lee, PhD ${ }^{3}$, Mark McClendon, PhD , Ryan Freshman, $B S^{1}$, Michael Schallmo, BS $S^{2}$, Samuel Stupp, PhD $D^{4}$ Erin $H s u, P h D^{2}$, Wellington Hsu, M.D. ${ }^{5}$

${ }^{1}$ Northwestern University Department of Orthopaedic Surgery, Chicago, IL, ${ }^{2}$ Northwestern University Department of Orthopaedic Surgery, Chicago , IL, ${ }^{3}$ Northwestern University Department of Materials Science and Engineering,., Chicago, IL, ${ }^{4}$ Northwestern University Department of Materials Science and Engineering, Chicago, IL, ${ }^{5}$ Northwestern Memorial Hospital, Chicago, Illinois

Background/Introduction: Advances in biologics and bone graft substitutes have improved spine fusion rates; however, there still exists a need for a product that elicits high fusion rates with minimal adverse effects. Recombinant human bone morphogenetic protein-2 (rhBMP-2) applied onto absorbable collagen sponges (ACS) promotes fusion rates of $>90 \%$ in humans. However, the supraphysiologic dose required when rhBMP-2 is utilized with this carrier can lead to serious complications. In previous work, we evaluated nanofiber scaffolds composed of peptide amphiphiles (PA), which localize BMP-2, thereby reducing the requisite dose of exogenous growth-factor for successful fusion. The purpose of the current study was to develop a novel growth-factor carrier that would enhance the properties of our PA nanoscaffold while reducing the amount of rhBMP-2 necessary to achieve fusion. We hypothesized that a collagen slurry vehicle would optimize assembly of a carboxyl-rich (E3)PA nanogel, yielding a malleable paste that could be used to fill bone defects and potentially reduce the exogenous growth-factor necessary to achieve arthrodesis.

Materials/Methods: Female Sprague-Dawley rats and New Zealand white rabbits underwent L4-L5 posterolateral spine fusion (PLF) procedures with placement of collagen slurry +/-E3PA or ACS alone. Scaffolds were preloaded with saline or rhBMP2 (100ng/rat or $60 \mu \mathrm{g} / \mathrm{rabbit})$. Bone regeneration and fusion were assessed using radiographs, manual palpation-based fusion scoring, microCT imaging (rats), and histology (rats).

Results: Preloading E3PA-collagen slurry with 100ng rhBMP2 in rats elicited a significantly higher mean fusion score relative to equivalently pre-loaded ACS $(\mathrm{p}<0.001)$ or control slurry $(\mathrm{p}<0.001)$. Successful fusion was seen in $100 \%$ of animals treated with E3PA-collagen slurry +100ng rhBMP-2. This was significantly higher than fusion rates of equivalently pre-loaded ACS $(0 \%)$ and control slurry $(8 \%)$. Similarly, fusion rates in rabbits treated with E3PA-collagen slurry $+60 \mu \mathrm{g}$ rhBMP-2 (100\%) were significantly higher than equivalently pre-loaded ACS $(50 \%)$ $(\mathrm{p}<0.05)$.

Discussion/Conclusion: Multiple groups have established 
$10 \mu \mathrm{g}$ rhBMP-2 applied on ACS as a $100 \%$ fusion positive control in the rodent model. Our data suggests that E3PA-collagen slurry can effectively reduce the requirement for rhBMP-2 by a factor of 100 relative to ACS, the current FDA-approved carrier. E3PA also served as an improved rhBMP-2 carrier in the rabbit PLF model.

Paper \#42. Comparative Analysis of Fusion Rates and Associated Complications in Patients With and Without rhBMP2 Two Years After Undergoing Anterior Lumbar Interbody Fusion

Jim Youssef, MD; Douglas Orndorff. MD, Sue Lynn Myhre, PhD, Cacia Pope, BS, Tara Faye, BS

Spine Colorado, Durango, CO

Background/Introduction: To perform a comparative analysis of fusion rates and associated complications in patients with and without rhBMP2 two years after undergoing Anterior Lumbar Interbody Fusion (ALIF). Superior fusion outcomes have been observed for ALIF surgeries when rhBMP2 was incorporated. However, compared to those without rhBMP2, there are questions of whether or not the potential benefits outweigh the risk of complications (retrograde ejaculation, subsidence and urogenital problems) that can be anticipated.

Materials/Methods: Data was retrospectively collected for 203 ALIF patients with or without rhBMP2 from 2008-2012. The analysis included the amount of rhBMP2 used, time to fusion, and associated complications. Fusion statuses were assessed via $\mathrm{x}$-ray and $\mathrm{CT}$ at standard of care visits. The majority of patients underwent a single level fusion $(\mathrm{N}=188)$, while the remaining patients experienced a two-level fusion $(\mathrm{N}=15)$.

Results: rhBMP2 was utilized in varied amounts for 110 patients, and allograft alone was employed in 93 patients. Of the latter, 26 experienced complications: pseudarthrosis $(n=7)$, radiculopathy $(n=3)$, adjacent segment disease (ASD; $n=2)$, non-index level foraminal narrowing $(n=3)$, and others $(n=12)$. Of the rhBMP2 cohort, 17 had complications: ASD $(n=3)$, radiculopathy $(n=2)$, canal stenosis $(n=1)$, erectile dysfunction $(n=1)$, and others $(10)$, with no pseudarthrosis. On average, those that received 1.4-2.8 cc $(\mathrm{n}=64)$ of rhBMP2 fused by 12.4 months, while those that received 5.6-8.0 $\mathrm{cc}(\mathrm{n}=31)$ fused by 12.8 months $(\mathrm{p}>0.05)$.

Discussion/Conclusion: Between the two cohorts, those who did not receive rhBMP2 had nearly twice as many complications as those with rhBMP2. These results support previous studies showing the arthrodesis advantages of rhBMP2 and also demonstrated fewer complications, which lends additional support for the use of rhBMP2. In addition, the amount of rhBMP2 does not seem to influence the rate of fusion nor increase the risk of complications in this patient population. More well designed studies are needed to confirm our finding that the amount of rhBMP2 does not impact the quality of fusion and patient outcomes.

Paper \#43. Regeneration of Intervertebral Disc using Human Umbilical Cord-derived Mesenchymal Stem Cells

\section{Mick Perez-Cruet, $M D$}

\section{, Southfield , Michigan}

Background/Introduction: Degenerative disc disease (DDD) is caused due to the loss of nucleus pulposus (NP) of the intervertebral disc (IVD) and is commonly responsible for chronic back pain disorders. Chrondrocytes represent the primary cell type seen within the NP and may be responsible for producing and maintaining the extra-cellular water matrix (i.e. glucosaminoglycans (GAG)) that keep the disc hydrated. Currently, no biologic disc regenerative treatment is available for this debilitating disease that restores the integrity of the NP completely. Human umbilical cord-derived mesenchymal stem cells (UC-MSCs) with fewer HLA mismatches and immunosuppressive properties are an attractive and promising source for cell therapy to restore the IVD and alleviate pain. In this study, we investigated the therapeutic potential of UC-MSCs and chondroprogenitor cells (i.e., UC-MSCs differentiated in cell culture into chondrocytes) in an IVD degeneration rabbit model.

Materials/Methods: A DDD rabbit model described previously was injected with PKH26-labeled UC-MSCs or chondroprogenitor cells into degenerated disc (L2-3 or L4-5), L3-4 served as a control. Eight weeks post-implantation, rabbits were euthanized and harvested discs were subjected to glycosaminoglycan (GAG) content analysis, extracellular matrix composition and expression of NP specific genes using biochemical assay, immunohistological staining, and qRT-PCR, respectively. In-vitro analysis was also conducted using magnetic resonance imaging pre and postimplantation of stem cells.

Results: Both UC-MSCs and chondroprogenitor cells Implanted into the DDD survived and integrated into the NP region. Differentiated chondroprogenitor cells maintained structural and cellularity integrity of the NP much better than did the undifferentiated UC-MSCs. In addition, discs implanted with chondrogenic derivatives had higher GAG content and expressed higher levels of NP specific markers such as SOX9, COL2, ACAN, FOXF1, and KRT19. MRI showed improved water content in disc implanted with chondroprogenitor cells then in experimental nonimplanted disc and those implanted with UC-MSCs.

Discussion/Conclusion: Implanted umbilical cord blood stem cells survived, dispersed, and integrated into the disc. However, chondroprogenitor cells derived from UC-MSCs showed greater potential to regenerate the NP. The results of this study demonstrates the promising therapeutic potential of umbilical cord blood MSCs derived chondroprogenitor cells and provides basis for translational applications to treat DDD.

Paper \#44. Notochordal Cell-Based Therapeutics Can Regenerate the Degenerative Disc

Ajay Matta, PhD $^{l}$, Muhammad Karim,DVMMSC MPhil(Hons) $)^{2}$; William Erwin, $D C P h D^{3}$

${ }^{1}$ Krembil Research Institute, Toronto, Ontario, ${ }^{2}$ Krembil Research Institute, Toronto, Ontario, ${ }^{3} \mathrm{Krembil}$ Research Institute/ Toronto Western Hospital, Toronto, Ontario

Background/Introduction: Degenerative disc disease (DDD) currently has no biological therapy capable of influencing the course of the disease. However the non-chondrodystrophic (NCD) or "mongrel" dog that retains its population of notochordal cells (unlike humans) does not develop DDD if at all, until much later in life. Here we demonstrate that NCCM is capable of regenerating the degenerative disc in a pre-clinical animal model of DDD.

Materials/Methods: We used a 26-gauge needle and image guidance to develop DDD in a pre-clinical rodent model and characterized the degenerative cascade from healthy through 10-weeks by analyzing the IVD NPs until 6-weeks post injury. Meanwhile we generated notochordal cell conditioned medium (NCCM) from the intervertebral disc (IVD) nucleus pulposus (NP) of NCD canines using protein deficient tissue culture medium. The NCCM was concentrated using molecular weight cut-off filters and 8ul of NCCM diluted 50:50 with PBS was injected into 4 disc/ animal (4-weeks post injury) and in control animals we injected 8ul PBS. 6-weeks post injury (10 weeks total) we sacrificed the animals and assayed the IVDs. 
Results: DDD induced in the pre-clinical rat-tail IVD NP proceeds in P38 MapKinase-dependent fashion and is associated with pronounced inflammatory events culminating at 10 -weeks post injury when the formerly healthy, notochordal cell-rich NP degenerates to become a fibrocartilagenous phenotype. At 6-weeks post injury, there is almost a complete loss of notochordal and stem cells in the injured groups with marked reduction in collagen type II and aggrecan. However, the NCCM groups retain/ regenerate a notochordal and stem-cell rich NP with abundant aggrecan and collagen type II immunostaining that is significantly downgraded in the degenerative PBS-treated discs. Furthermore, within the NCCM injected discs the expression of Cox2, MMP-13 and MMP-3 is undetectable whereas the PBS injected control discs demonstrate abundant Cox 2 and MMP expression demonstrating the marked anti-inflammatory, pro-anabolic and anti-catabolic activity of NCCM.

Discussion/Conclusion: The components of NCCM can regenerate/restore the needle puncture injured degenerative disc in a rodent model of DDD. The necessary and sufficient factors contained within NCCM could be used in a minimally invasive molecular therapy for the treatment of degenerative dis disease.

Paper \#45. Cytokine Receptor Knockdown via CRISPR/ Cas9 for Treatment of Disc Herniation-Associated Pain

\author{
Jing Han, $B A^{l}$, Liufang Jing, $M S^{2}$, Jun Chen, Ph.D. ${ }^{1}$, Lori \\ Setton, Ph.D. ${ }^{2}$
}

${ }^{1}$ Duke University, Durham, NC, ${ }^{2}$ Washington University in St. Louis, St. Louis, MO

Background/Introduction: Intervertebral disc (IVD) herniation is frequently associated with radicular pain mediated by both mechanical compression of nerve roots and presence of proinflammatory cytokines (e.g. TNF- $\alpha$, IL17A). Previously, biologics have been explored to antagonise these cytokines with application to neuroinflammation and symptom development. Recent advances in the use of the novel genome engineering tool Clustered Regularly Interspaced Short Palindromic Repeats interference (CRISPRi) enable highly specific knockdown of target proteins using guide RNAs (gRNA) directed to loci of interest.

Materials/Methods: We developed CRISPRi-gRNA constructs to knockdown receptors to TNF- $\alpha$ and IL17 to abrogate development of neuropathic pain. Guide RNA sequences targeting rat TNF receptor type I (TNFRI) and IL17 receptor type A (IL17RA) promoter regions were selected based on appropriate protospacer adjacent motifs (crispr.mit.edu), cloned into plasmids under control of the U6 promoter, and screened for effective knockdown via qRT-PCR. The gRNA showing greatest knockdown was cloned into lentiviral (LV) vectors to facilitate single-vector co-expression with dCas9-KRAB-GFP. Rat PC12 cells and primary DRG neurons were transduced with TNFRI and IL17RA LV CRISPRi vectors and qRT-PCR was performed to identify knockdown. Transduction efficiency was measured via flow-cytometry for GFP expression (Accuri C6). Functional effect was confirmed by quantifying TNF- $\alpha$ - and IL17A-induced ERK phosphorylation (PerkinElmer AlphaLISA).

Results: Selected CRISPRi-gRNA constructs were able to knockdown $78 \%$ and $95 \%$ of mRNA for TNFRI and IL17RA, respectively, in rat PC12 cells. Flow cytometry in primary rat DRG cells demonstrated $60.3 \%$ transduction efficiency. p-ERK levels significantly decreased (84\% for TNFRI and $93 \%$ for IL17RA at 15 minutes) in response to TNF- $\alpha$ challenge after both TNFRI and IL17RA CRISPRi knockdown in PC12 cells. This suggests an ability to interfere with TNF- $\alpha$-induced cell signalling that can contribute to painful neuropathy.
Discussion/Conclusion: CRISPRi knockdown of TNFRI and IL17RA can be used to modulate cellular response to inflammatory challenges in neuronal cells, DRG neurons and cells. Despite incomplete transduction efficiency, knockdown of either receptor expression inhibits ERK, a proximal effector in the intracellular signalling pathway associated with pain development. Future work will evaluate functional assessments of CRISPRi-mediated knockdown in DRGs in vitro and in vivo following cytokine challenge or IVD herniation.

RF Paper 1. Frailty is Predictive of Adverse Postoperative Events in Patients Undergoing Lumbar Fusion $\underline{B S}^{2}$

Dante Leven, DO ${ }^{l}$, Samuel Cho, $M D^{l}$; John Di Capua, MHS,

${ }^{1}, \mathrm{NY}, \mathrm{NY},{ }^{2}$ Icahn School of Medicine at Mount Sinai, New York, New York

Background/Introduction: Lumbar fusion is a common surgical procedure for elderly patients with spinal pathology though high complication rates are reported. Frailty is a measure of health status and can be used to predict postoperative complications. The $\mathrm{mFI}$ has been validated in several surgical cohorts, though has not been studied in patients undergoing posterior or transforaminal lumbar interbody fusion (PLIF or TLIF).

Materials/Methods: The American College of Surgeons National Surgical Quality Improvement Program (ACS NSQIP) database is a large multicenter clinical registry that collects over 200 perioperative variables from about 400 hospitals nationwide. The study cohort was patients over 18 years old who underwent PLIF or TLIF between 2005 and 2012. The previously described $\mathrm{mFI}$ was calculated and univariate and multivariate logistic regression analysis were used to analyze risk factors associated with morbidity, mortality and adverse postoperative events.

Results: 6094 patients met inclusion criteria. The mean mFI was $0.087(0-0.545)$. Increasing $\mathrm{mFI}$ score was associated with increased complications, reoperations, prolonged length of stay (LOS), and morbidity $(\mathrm{p}<0.05)$. As the $\mathrm{mFI}$ score increased from 0.27 (3/11 variables present) to $\geq 0.36(4 / 11)$ the rate of any complication increased from $26.8 \%$ to $35 \%$ ( $\mathrm{p}<0.0001$ ), sepsis $2.4 \%$ to $5.2 \%(\mathrm{p}<0.0001)$, wound complications $4.4 \%$ to $6.5 \%$ $(\mathrm{p}<0.0001)$, unplanned readmissions $4.7 \%$ to $20 \% \quad(\mathrm{p}=0.02)$, urinary tract infection (UTI) $4.1 \%$ to $10.4 \%(\mathrm{p}<0.0001)$. An mFI of $>/=$ to 0.36 was an independent predictor of any complication (OR 2.2,1.3-3.7), sepsis (OR 6.3,1.8-21), wound complications (OR 2.9,1.1-8.2), prolonged LOS (OR 2.3,1.4-3.7) and readmission (OR 4.3,1.5-12.7).

Discussion/Conclusion: Patients with higher $\mathrm{mFI}$ scores $(\geq 4 / 11$ variables) are at a significantly higher risk of major complications, readmissions and prolonged LOS following lumbar fusion.

RF Paper 2. The Effect of Coagulopathies on Perioperative Complications and Clinical Outcomes in Patients Treated with Posterior Lumbar Fusion

Varun Puvanesarajah, $B S^{1}$, Amit Jain, $M D^{2}$, Adam Shimer, $M D^{l}$, Francis Shen, $M D^{3}$, Hamid Hassanzadeh, $M D^{l}$

${ }^{1}$, Charlottesville, VA, ${ }^{2}$, Baltimore, MD, ${ }^{3}$, Charlottesville, Virginia

Background/Introduction: Both hypercoagulable states and coagulopathies are not uncommon conditions that have the potential to significantly alter perioperative patient management. 
However, there are few studies that document the added risk of medical complications following spine surgery in these patient populations. The objective of this study is to compare complication rates following 1-2 level lumbar spine surgery in patients with primary hypercoagulable states and coagulopathies.

Materials/Methods: The PearlDiver database (2005-2012) was utilized to determine perioperative complication rates in patients with primary hypercoagulable states and coagulopathies who underwent primary 1-2 level posterolateral lumbar spine fusion. Control cohorts without specific hematologic disorders were matched by demographics and comorbidities. Ninety-day complication rates were determined, along with revision rates at one and two years. When considering complication rate comparisons between matched cohorts, $\mathrm{p}<0.005$ was considered significant.

Results: In total, 746 patients with coagulopathies and 2,753 patients with primary hypercoagulable states were selected. Matched control cohorts contained 74,879 and 54,007 patients, respectively. Hypercoagulable patients had significantly increased rates of medical complications (OR 1.4), infections (OR 1.6), and venous thromboembolisms (OR 9.0) during the three months following spine surgery and same-day transfusions (OR 1.2) when compared to matched controls $(\mathrm{p}<0.001)$. Patients with von Willebrand disease or hemophilia had increased rates of 3-month infections (OR 2.3) and transfusion (OR 2.0) when compared to a matched control group $(p<0.005)$. One- and two-year revisions rates were not significantly higher for either pathologic cohort when compared to matched controls.

Discussion/Conclusion: Both primary hypercoagulable states and coagulopathies increase the rate of transfusions and infections within three months when compared to matched controls. Increased rates of venous thromboembolisms and medical complications are also observed following lumbar spine surgery in patients with primary hypercoagulable states. The increased risk of complications in these two cohorts must be considered when evaluating patients with hematological abnormalities for lumbar spine surgery.

RF Paper 3. National Trends for Primary and Revision Lumbar Disc Arthroplasty Throughout the United States from 2005-2013

Comron Saifi, $M D^{l}$, Alex Ha, $M D^{2}$, Alejandro Cazzulino, $B A^{l}$, Lawrence Lenke, $M D^{1}$, Ronald Lehman, $M D^{3}$

\section{${ }^{1}$, New York, NY, ${ }^{2}$, New York , NY,${ }^{3}$, New York, NY}

Background/Introduction: Given the increasing focus on health care utilization and value-based care, it is essential to determine the number of primary and revision lumbar disc arthroplasty (LDA) per year throughout the United States. Additionally patient demographics and economic data associated with primary and revision LDA are critical to understanding and improving health care utilization.

Materials/Methods: The data utilized in this study was collected between 2005-2013 across 44 states from the National Inpatient Sample (NIS) database on patients who underwent either primary LDA (ICD-9 CM 84.65) or revision LDA (ICD-9 CM 84.68). Demographic and economic data were obtained which included the annual number of surgeries, age, sex, total charges, total costs, length of stay (LOS), insurance type, location, and frequency of routine discharge. The NIS database represents a $20 \%$ sample of discharges from U.S. hospitals, excluding rehabilitation and long-term acute care hospitals, which is weighted to provide national estimates.

Results: The total number of primary LDA has progressively decreased $86 \%$ from 3,059 in 2005 to 420 in 2013. The mean total cost, including hospital and physician related costs, of LDA increased 33\% from 2006 to 2013 from $\$ 17,747$ to $\$ 23,804$ with a mean of $\$ 22,310$ per case over the nine year study period. The mean LOS decreased from 2.8 days in 2005 to 2.3 days in 2012. The mean routine discharge of $90 \%$ did not vary significantly based on year. The number of revision procedures similarly declined $65 \%$ from 205 in 2007 to 71 in 2011. The mean national revision burden was $10 \%$ (range $6 \%$ to $24 \%$ ). The mean cost of revision LDA ranged from $\$ 12,752$ to $\$ 22,282$ over the study period. The mean LOS after the revisions was 3.4 days.

Discussion/Conclusion: Over the course of this study (20052013), primary and revision LDA progressively declined in the U.S. by $86 \%$ and $65 \%$, respectively. The total costs for primary LDA increased by $33 \%$ between 2006 and 2013. Over this time period the national revision burden throughout the United States has risen significantly from $6 \%$ to $24 \%$.

RF Paper 4. National Trends for Primary and Revision Anterior Lumbar Interbody Fusion Throughout the United States from 2004-2013

Comron Saifi, $M D^{1}$, Alex $H a, M D^{2}$, Alejandro Cazzulino, $B A^{l}$, Lawrence Lenke, $M D^{I}$, Ronald Lehman, $M D^{3}$

\section{${ }^{1}$, New York, NY, ${ }^{2}$, New York , NY,${ }^{3}$, New York, NY}

Background/Introduction: For this study our group hypothesized that the number of anterior/lateral lumbar interbody procedures including anterior lumbar interbody fusions (ALIF), extreme lateral interbody fusion (XLIF), and direct lateral interbody fusion (DLIF) has increased over the past decade. Patient demographics and economic data associated with primary and revision anterior/lateral lumbar interbody cases is critical to understanding and improving optimal health care utilization.

Materials/Methods: The data utilized in this study was collected between 2004-2013 across 44 states from the National Inpatient Sample (NIS) database on patients who had undergone ALIF/XLIF/DLIF (ICD-9-CM-81.06) or revision ALIF/XLIF/ DLIF (ICD-9-CM-81.36). Demographic and economic data including, annual number of surgeries, age, sex, total charges, total costs, length of stay (LOS), insurance type, location, and frequency of routine discharge were obtained. The NIS database represents a $20 \%$ sample of discharges from U.S. hospitals, which is weighted to provide national estimates.

Results: An estimated 319,730 and 15,938 patients underwent primary ALIF/XLIF/DLIF and revision ALIF/XLIF/DLIF procedures, respectively, throughout the U.S. during the study period. The total number of these anterior/lateral lumbar interbody operations has progressively increased $69 \%$ from 20,900 to 35,195 over the ten-year study period. The mean total cost, including hospital and physician related costs, of these surgeries increased $26 \%$ from $\$ 30,561$ to $\$ 38,614$ over the same period. The mean LOS decreased from 5.0 to 3.7 days. The mean routine discharge of $74 \%$ did not vary significantly based on year. The mean national revision burden, the ratio of revision procedures to the sum of primary and revision procedures, was $4.8 \%$. The mean cost of revision anterior/lateral lumbar spine interbody surgeries was $\$ 38,898$. The mean LOS after the revisions was 5.0 days.

Discussion/Conclusion: Over the past ten years (2004-2013) there has been a progressive increase in primary anterior/lateral interbody lumbar surgeries by $69 \%$ throughout the United States as well as an increase in total costs for primary anterior/lateral interbody surgeries by $26 \%$. This is likely driven in part by an increasing interest in XLIF procedures. LOS has progressively decreased over the same time period to 3.7 and 4.4 days for primary and revision cases, respectively. The mean national revision burden has remained constant at $4.8 \%$. 


\section{RF Paper 5. Missing Data May Invalidate Spine Database Studies}

Bryce Basques, $M D^{l}$, Andre Samuel, BBA ${ }^{2}$, Adam Lukasiewicz, $M S c^{2}$, Matthew Webb, $A B^{2}$, Daniel Bohl, MD, MPH $H^{3}$, Junyoung Ahn, $B S^{l}$, Kern Singh, $M D^{l}$, Jonathan Grauer, $M D^{4}$

${ }^{1}$ Rush University Medical Center, Chicago, IL, ${ }^{2}$ Yale School of Medicine, New Haven, CT, ${ }^{3}$ Rush University Medical Center, New Haven, CT, ${ }^{4}$, New Haven, CT

Background/Introduction: National databases are being increasingly used for spine research, however missing data is a significant limitation that has received little attention. This study uses spine surgery patients from the American College of Surgeons National Quality Improvement Program (ACS-NSQIP) database to illustrate how missing data can significantly skew results of these studies.

Materials/Methods: Patients who underwent spine surgery between 2005 and 2013 were identified from the ACS-NSQIP database. Demographics, comorbidities, and perioperative lab values were tabulated for each patient and the percent of missing data was noted for each variable. These variables were tested for association with "any adverse event" using two separate multivariate regressions that used the two most common treatments for missing data. In the first regression, patients with any missing data were simply excluded. In the second regression, missing data was treated as a negative, or "reference" value. The results of these regressions were compared in order to determine how the different treatments of missing data could affect the results of spine studies using the ACS-NSQIP database.

Results: A total of 88,471 spine surgery patients were identified. Nineteen comorbidity variables had $65.54 \%$ missing data, including smoking history, alcohol use, and myocardial infarction. Multivariate logistic regressions using the two different treatments of missing data (Table 1) found vastly different significant risk factors for adverse events: out of 33 risk factors found to be significantly associated with adverse events in either analysis, only $16(48.4 \%)$ of these risk factors were common between the two regressions.

Discussion/Conclusion: Missing data was common for spine surgery patients in the ACS-NSQIP. Importantly, 19 comorbidity variables had $65.54 \%$ missing data, as they are now only collected at certain ACS-NSQIP participating sites. This is not made clear in the basic user manual distributed with the dataset and researchers must be diligent when using this data. As shown, differing treatments of missing data can significantly affect the results of spine studies using the ACS-NSQIP. This study raises significant questions about using spine patients from the ACS-NSQIP and it is important for researchers to be aware of these limitations when designing, performing, and evaluating such investigations.

RF Paper 6. The Clinical Spectrum of PROMIS Physical Function Scores Over Time in Patients with Operative Lumbar Pathology

Stephen Pehler, $M D^{l}$, Brandon Lawrence, $M D^{2}, W$. Ryan Spiker, $M D^{2}$, Yue Zhang, $P h D^{3}$, Darrel Brodke, $M D^{2}$

${ }^{1}$ University of Utah, Salt Lake City, Utah, ${ }^{2}$, Salt Lake City, Utah, ${ }^{3}$ University of Utah, Salt Lake City, Utah

Background/Introduction: With a continually increasing focus on Value in today's healthcare economy, Patient reported outcome measures provide critical information on assessing the effectiveness of care delivered. Patients seek care at a spine center generally with two goals in mind: to increase physical function, and decrease pain. Prior studies have identified problems with legacy scores (NDI, ODI, SF-36, etc) in measuring patient outcomes. PROMIS physical function domain scores have been shown to be valid, accurate, and have low floor and ceiling effects in the spine patient population. What this study attempts to describe and understand is how PF CAT scores vary over time, from preoperative evaluation to the post-operative period, with treatment.

Materials/Methods: Retrospective review of PRO scores from 2013 to 2015 were collected at a single university spine center. Patients were included in the study if they were over the age of 18 and had a complaint of primary operative lumbar pathology. PRO scores were collected at each visit and aggregated over time from preoperative treatment to 15 months in the postoperative course.

Results: A total of 870 unique patients were followed during the collection period. A linear mixed effect model and linear mixed quantile regression model were fitted, respectively. The predicted PF CAT scores and PF CAT quantiles were mapped over time at intervals from the preoperative period to 15 months postop (attached figure 1 and 2)

Discussion/Conclusion: The PROMIS PF item bank has been previously shown to be a valid and accurate method of assessing patient reported outcomes in the spine patient population. To our knowledge, this is the first study to describe the overall trend of PF CAT scores over time, as well as the responsiveness of PROMIS scores, to treatment. Starting in the preoperative setting, this data can potentially assist in educating patients on their expected progress with treatment of lumbar pathology. Also, this data provides a unique perspective for a patient's current state as it relates to other patients with similar pathology at different stages of treatment.

RF Paper 7. The Effect of Bone Mineral Density on Proximal Junctional Failure in Thoraco-Lumbar Fusion

Tarush Rustagi, $M D^{l}$, Richard Tallarico, $M D^{l}$, Nikhil Thakur, $M D^{l}$, Mike Sun, $M D^{l}$, Ian Madom, $M D^{l}$; Henaku Yirenkyi, $\mathrm{MD}^{2}$, William Lavelle, $M D^{\prime}$ NY

${ }^{1}$, Syracuse, NY, ${ }^{2}$ SUNY Upstate Medical University, Syracuse,

Background/Introduction: Proximal junctional failure (PJF) can be a catastrophic complication associated with adult spinal deformity surgery. Due to regional sclerosis, spinal DEXA scan scores have been reported as flawed and unreliable. Recently, investigators have reported using CT scans as surrogates in place of spinal DEXA scores. We investigated if CT based assessments of bone mineral density (BMD) can predict the possibility of PJF.

Materials/Methods: Records and data of spinal deformity patients $(n=26)$ operated on (2007-2012) were retrospectively reviewed from a single center. All patients had constructs involving fusions to the pelvis and encompassing at least five lumbar levels. Indications included flat back, positive sagittal balance and degenerative lumbar scoliosis. Each underwent preoperative standing x-rays and CT scans. Patients divided into groups with PJF as either having progression in kyphosis over 10 degrees (proximal junctional kyphosis; PJK) or having fracture at proximal level. Three groups created: Group 1 patients with no PJF ( $n=16)$; Group 2 with PJK $(n=6)$; and Group 3 proximal level fracture $(n=4)$. CT based BMD was calculated by defining a region of interest within the vertebral body in sagittal plane. Hounsfield units (HU) measured within this region of interest. The vertebra at most cranial end of final construct defined as upper instrumented vertebra (UIV). Measurements made at UIV, UIV + 1(proximal), and UIV -1(distal).

Results: Comparison between patients with and without 
proximal junctional failure shows that 10/26 had failure. T5-9 was most proximal levels for PJF (50\%) and without PJF (62.5\%). There was no significance comparing mean BMD between Group 1 and 2, but between Group 1 and Group 3, UIV +1 showed significance $(\mathrm{p}=0.041)$. Mean proximal kyphosis was 13.8 degrees (10-32 degrees). Four patients with proximal level fracture, two involved vertebral body with implant back out and two had endplate fracture.

Discussion/Conclusion: BMD measured from a CT scan was an effective tool in planning proximal extent of fusion in adult deformities. BMD values of less than $130 \mathrm{HU}$ at planned upper instrumented vertebra was a risk factor for a proximal junctional fracture. For proximal junctional kyphosis, our study found no statistical significance.

RF Paper 8. Diabetes Mellitus as a Predictor for Adverse Outcomes in Adult Spinal Deformity Cases

John Di Capua, MHS, BS ${ }^{1}$, Sulaiman Somani, BS $S^{1}$, Joung Heon Kim, $B S^{2}$, Nathan Lee, $B S^{1}$, Parth Kothari, $B S^{1}$, Samuel Cho, $M D^{3}$

${ }^{1}$ Icahn School of Medicine at Mount Sinai, New York, New York, ${ }^{2}$ Icahn School of Medicine at Mount Sinai, New York, NY, ${ }^{3}, \mathrm{NY}, \mathrm{NY}$

Background/Introduction: Diabetes mellitus is a prevalent chronic disease with extensive secondary sequela and may influence the incidence of adverse outcomes in elective spinal deformity cases.

Materials/Methods: This was a retrospective analysis of prospectively collected data from the National Surgical Quality Improvement Program (NSQIP) database between 2010 and 2014 for patients undergoing Adult Spinal Deformity (ASD) Surgery. The NSQIP database collected 135 patient demographic, comorbidity, operative condition and postoperative outcome variables up to 30 days following the procedure. Patients were categorically divided into 3 classes by diabetes status: nondiabetic, non-insulin-dependent diabetes mellitus (NIDDM) and insulin dependent diabetes mellitus (IDDM). The groups were compared using chi-square tests and multivariate logistic regression models were employed to determine the effect of diabetes on postoperative outcomes.

Results: 5,338 (91.7\%) cases met the inclusion criteria for this study. 4,548 (85.2\%) patients were non-diabetic, $540(10.1 \%)$ patients had NIDDM and $250(4.7 \%)$ patients had IDDM. Multivariate logistic regression revealed diabetic status to be an independent risk factors, IDDM and NIDDM compared to Non Diabetic, in length of stay $(\mathrm{OR}=1.55,(1.15,2.09) \mathrm{p}$-value $=0.0042$; $\mathrm{OR}=1.27, \quad(1.02,1.58) \quad \mathrm{p}$-value $=0.0337)$, all postoperative complications $(\mathrm{OR}=1.27,(0.93,1.74) \mathrm{p}$-value $=0.1300 ; \mathrm{OR}=1.27$, $(1.01,1.58) \mathrm{p}$-value $=0.0374)$, postoperative UTI $(\mathrm{OR}=0.85,(0.34$, 2.15) $\mathrm{p}$-value $=0.7369 ; \mathrm{OR}=1.87,(1.14,3.05) \mathrm{p}$-value $=0.0124)$ and postoperative cardiac complications $(\mathrm{OR}=4.05,(1.72,9.51)$ $\mathrm{p}$-value $=0.0013 ; \mathrm{OR}=1.95,(0.83,4.62) \mathrm{p}$-value $=0.1273)$.

Discussion/Conclusion: Diabetes mellitus is significantly associated with a variety of adverse postoperative outcomes including increased length of stay, postoperative 30 day all complication rate, postoperative 30 day UTI and 30 day postoperative cardiac complications for patients undergoing elective ASD procedures.
RF Paper 9. Transcranial Motor-Evoked Potentials for Prediction of Postoperative Neurologic Deficit Following Surgery for Thoracolumbar Scoliosis

Sam Miller, $B S^{l}$, Sean Donegan, $B A^{2}$, Adam Eltorai, $B A^{3}$, Niesha Voigt, BA, Jason Machan, $P h D^{4}$, Alan Daniels, $M D^{5}$, Teena Shetty, $M D^{6}$

${ }^{1}$, Providence, RI, ${ }^{2}$ Hospital for Special Surgery, New York, New York, ${ }^{3}$ Warren Alpert Medical School of Brown University, Providence, RI, ${ }^{4}$ Department of Biostatistics, Warren Alpert Medical School of Brown University and Rhode Island Hospital, Providence, RI, ${ }^{5}$ Department of Orthopaedics, Division of Spine Surgery- Adult Spinal Deformity Service, Warren Alpert Medical School of Brown University, Providence, Rhode Island, ${ }^{6}$ Department of Neurology, Hospital for Special Surgery, New York, New York

Background/Introduction: TcMEPs have become an effective tool for monitoring the descending motor pathway during scoliosis surgery. By comparing potentials measured before and after correction, data can be provided to the surgeon that may inform maneuvers to prevent postoperative functional loss in distal muscles. There is currently no consensus on which muscles should be monitored for scoliosis surgeries. By comparing the efficiency of different muscle combinations, the least invasive monitoring protocol that will yield the best predictive insight may be determined.

Materials/Methods: A retrospective review of 118 patients who had TcMEP monitoring during surgical correction of thoracolumbar scoliosis at Hospital for Special Surgery between January 2008 and May 2015 was conducted. 20 of these patients had a postoperative neurologic consult due to new onset lower extremity weakness- representing the cohort who had both TcMEP monitoring and postoperative neurologic consult. The remaining 98 patients were a control group consisting of a consecutive cohort who did not receive a postoperative neurologic consult. TcMEPs were recorded from vastus lateralis (VL), tibialis anterior (TA), peroneus longus (PL), aBductor hallucis (AH) and abductor pollicis brevis (APB) bilaterally. A TcMEP loss was defined as an intraoperative loss of a potential that did not resolve by the completion of surgery. Effectiveness of each muscle combination was evaluated independently and then compared to other combinations using Akaike Information Criterion (AIC) with lower values suggestive of a better quality statistical model.

Results: Monitoring of VL, TA, PL, AH and APB yielded a sensitivity of $85.00 \%$ and specificity of $90.82 \%$ (AIC $=63.98$ ). Monitoring of VL, TA, PL and AH yielded a sensitivity of $85.00 \%$ and specificity of $90.82 \%$ (AIC $=63.98$ ). Monitoring of VL, TA and PL yielded a sensitivity of $80.00 \%$ and specificity of $91.84 \%$ $(\mathrm{AIC}=67.84)$. TA, PL and AH yielded a sensitivity of $85.00 \%$ and specificity of $92.86 \%(\mathrm{AIC}=59.55)$. Monitoring of TA and PL yielded a sensitivity of $80.00 \%$ and specificity of $94.90 \%$ (AIC $=60.39$ ) (Table 1).

Discussion/Conclusion: Intraoperative monitoring of TcMEPs of the TA, PL, and AH provided the highest sensitivity and specificity and best predictive power for postoperative lower extremity weakness following thoracolumbar deformity surgery. 


\section{RF Paper 10. MRI for Flexible Sagittal Imbalance}

Akshay Sharma, $B A^{l}$; Sina Pourtaheri, $M D^{2}$, Jason Savage, M.D. ${ }^{3}$, Iain Kalfas, $\mathrm{MD}^{4}$, Edward Benzel, M.D. ${ }^{5}$, Thomas Mroz, M.D. ${ }^{5}$, Michael Steinmetz, $M D^{4}$

${ }^{1}$ Case Western Reserve University, School of Medicine, Cleveland Heights, OH, ${ }^{2}$ University of California, Los Angeles, Santa Monica, California, ${ }^{3}$ The Cleveland Clinic, Cleveland, Ohio, ${ }^{4}$ Cleveland Clinic, Department of Neurosurgery , Cleveland, OH, ${ }^{5} \mathrm{Cleveland}$ Clinic Foundation, Neurological Institute, Cleveland, $\mathrm{OH}$

Background/Introduction: Flatback deformity refers to the loss of normal lumbar lordosis and can result in sagittal imbalance. Surgical treatment of sagittal imbalance has been noted to improve quality of life. Scoliosis x-rays are the standard for assessing preoperative lumbar lordosis; however, particularly for flexible deformities, it is difficult to predict from these images the extent of intraoperative correction required, as a standing radiograph cannot predict the thoracolumbar alignment after intraoperative positioning.

Materials/Methods: The authors retrospectively identified 138 patients with sagittal imbalance, undergoing a total of 148 fusions of the lumbar spine. Demographic and clinical parameters were obtained. Pelvic incidence and lumbar lordosis were obtained from images preoperatively and at 6 months and 2 years followup. Health and quality of life outcomes were evaluated using the patient-reported EQ-5D, Pain Disability Questionnaire, and Patient Health Questionnaire (PHQ-9). Patient reported outcomes were obtained preoperatively and at 12 months follow-up.

Results: The patient sample included 42 men $(30 \%)$ and 96 women $(70 \%)$ with a mean age of 61 years. The mean difference was 2.9 degrees between the lumbar lordosis measured on supine MRI as compared to the intraoperative $x$-rays. The difference in lumbar lordosis between standing $\mathrm{x}$-rays and intraoperative $\mathrm{x}$-ray was 5.53 degrees. Flexible deformity was defined as 10 degrees or more of disparity in lumbar lordosis between the standing $\mathrm{x}$-rays and MRI. For flexible patients $(n=24)$, the lumbar lordosis measured on MRI more accurately predicted the intraoperative lumbar lordosis, as compared to the standing x-ray measurements $(\mathrm{p}=0.0009)$. At 12 months, the flexible cohort exhibited pre- to postoperative improvement that was significant for the EQ-5D ( $p$ $=0.0026)$, PDQ $(\mathrm{p}=0.0048)$, and PHQ-9 $(\mathrm{p}=0.018)$. However, a matched cohort of non-flexible deformities showed no statistically significant change in pre- to postoperative patient reported outcomes at 12 months.

Discussion/Conclusion: Within the flexible cohort, lumbar lordosis measured on MRI accurately predicted the intraoperative lumbar lordosis. The ability to preoperatively predict the intraoperative lumbar lordosis with positioning also helps with surgical planning and patient counseling regarding expectations and risks of surgery. Lastly, the flexible sagittal deformities had better clinical outcomes with surgery than the non-flexible, matched cohort.

RF Paper 11. Publication Rate of Abstracts Presented at LSRS Meetings is Greater Than that of Other Spine Meetings

Patawut Bovonratwet, $B S^{l}$, Matthew Webb, $A B^{l}$, Nathaniel Ondeck, B.S. ${ }^{2}$, Jonathan Grauer, $M D^{2}$

${ }^{1}$ Yale School of Medicine, New Haven, CT, ${ }^{2}$, New Haven, CT

Background/Introduction: Annual scientific meetings are a forum for disseminating new research findings prior to publication in peer-reviewed journals. A scientific meeting's quality may be directly correlated to the publication rate of abstracts presented.
Although publication rates from multiple orthopedic research conferences have been published in the literature, those of the Lumbar Spine Research Society (LSRS) have never been reported.

Materials/Methods: LSRS podium presentations from 2008 to 2012 and posters from 2010 to 2012 were reviewed; these are all the abstracts accepted by all LSRS meetings through 2012. For each abstract, we searched PubMed to determine if a full-text publication existed. Searches included the interval three years prior to and following a given meeting. First the title was searched. If the title was not found, each individual author's name was searched. If authors and content found on PubMed were similar to the abstract, then the abstract was considered published. Chisquared tests were used to compare LSRS publication rates to those of other spine meetings reported in the literature.

Results: From 2008 to 2012, a total of 332 podium and poster presentations were identified (See Table 1). The publication rate for podium presentations in 2008 was $51.5 \%$ and trended to increase through 2012 to $66.0 \%$. The publication rate for posters was lower, but showed a similar increasing trend. The overall publication rate for the lifetime of the meeting is $55.1 \%$ $(183 / 332)$. This is statistically greater than the publication rates that have been reported for North American Spine Society (40\%, $\mathrm{p}<0.001)$, Eurospine (Spine Society of Europe) $(37.8 \%, \mathrm{p}<0.001)$, International Society for the Study of the Lumbar Spine $(45 \%$, $\mathrm{p}=0.012$ ), and the Scoliosis Research Society (47\%, $\mathrm{p}=0.042$ ). The publication rate of the podium presentations at LSRS from $2008-2012(136 / 235,57 \%)$ was statistically similar to that of the Cervical Spine Research Society from 2007-2011 (211/321, $65.7 \%, \mathrm{p}=0.059)$.

Discussion/Conclusion: Our findings suggest that papers accepted for presentation at LSRS have a high likelihood of publication in a peer-reviewed journal on PubMed. Although LSRS is a relatively young society, the publication rate of LSRS meetings was greater than or similar to that of the meetings of other spine societies.

RF Paper 12. Do surgeons need to rescrub during operations that last longer than three hours?

Pooria Hosseini, M.D.. ${ }^{1}$, Gregory Mundis, M.D., ${ }^{2}$, Robert Eastlack, M.D., ${ }^{3}$, Jeff Pawelek, BS ${ }^{4}$, Stacie Nguyen, $M P H^{4}$

${ }^{1}$ San Diego Spine Foundation, San Diego , California , ${ }^{2}$ San Diego Spine Foundation, San Diego , California, ${ }^{3}$ Scripps Clinic, La Jolla , California , ${ }^{4}$ San Diego Spine Foundation, San Diego , California

Background/Introduction: Despite advances in infection control, surgical site infection (SSI) remains a substantial cause of morbidity and mortality in hospitalized patients. A variety of methods are being used to decrease SSI, however SSI can still cause complications especially in long operations ( $>3$ hours). There is evidence that the efficacy of the scrubbing material fades away after three hours. Hence, it can be postulated that the scrubbed surgeon's hands may become a progressive source of contamination during surgery. In addition, it has been shown that gloves do not provide a permanent physical barrier against microbes. We aimed to determine the level of surgeons' recurrent hand contamination after uninterrupted operations lasting more than three hours.

Materials/Methods: Three spine surgeons at the same facility used the same scrubbing technique and material. Exclusion criteria included procedures less than three hours, and procedures that required changing of both sets of gloves. Twenty consecutive spine surgeries meeting these criteria were included. Surgeons' hands were swabbed with sterile cotton tip applicators and $5 \mathrm{ml}$ sterile $75 \mathrm{mM}$ Phosphate Buffered Saline with $0.1 \%$ Triton-X (pH 
7.9) at the following time points: Prior to hand scrubbing (prescrub), immediately following hand scrubbing (post-scrub) and immediately following surgery (post-operative).

Results: All samples had zero colony count at post-scrub. None of the cases lasting 3-4 hours had colony growth post-operatively. Surgeries that were at least five hours had the post-operative colony count reach the same level or higher than the pre-scrub. The longer the operation, the higher the level of contamination post-operatively with a linear regression coefficient of 0.89 and $\mathrm{p}=0.005$. Fig 1 .

Discussion/Conclusion: Our results demonstrate that after four hours, a surgeon's hands become recolonized, despite preoperative scrubbing that successfully removed contaminants. In some cases, there was higher colony forming unit / $\mathrm{ml}$ after the procedure than before any scrubbing had occurred. Based on these findings, consideration should be given to rescrubbing during the course of surgical procedures extending beyond four hours.

RF Paper 13. Anatomic Considerations of Extraforaminal Lumbar Interbody Fusion: A Cadaveric Study

Abhishek Kumar, MD, FRCSC ${ }^{1}$, Sheeraz Qureshi, $M D, M B A^{2}$, Robert Merrill, $B S^{2}$

${ }^{1}$ Mount Sinai Medical Center, New York , NY, ${ }^{2}$ Mount Sinai Medical Center, New York, NY

Background/Introduction: While transforaminal and lateral remain the workhorse minimally invasive approaches to access the lumbar intervertebral disc, there has been recent interest in a new extra-foraminal lumbar interbody fusion (ELIF) intended to reduce procedural complications and morbidity. The purpose of this cadaveric study was to determine the safety of the ELIF approach with respect to the exiting nerve roots and the lumbar plexus.

Materials/Methods: A cadaveric study was done using live fluoroscopy to pass needles lateral to the facet joint into the lumbar intervertebral discs. With the cadaver in prone position the $\mathrm{C}$-arm was centered over the disc of interest, aligned perpendicular to the superior endplate of the inferior vertebral body. The C-arm was then rotated so that the anterior margin of the superior articular process (SAP) of the inferior vertebral body bisected the superior endplate. Maintaining a trajectory inline with the fluoroscopy beam, three needles were passed into the disc, all equally spaced along the accessible area. The process was repeated at each disc level from L1 body to L5 body bilaterally on 2 cadavers, totaling 16 passes for each trajectory. After the needles were placed, the cadaver was dissected to expose and measure the proximity from each pin to exiting nerve root as well as the lumbar plexus.

Results: The overall average distance from the medial, middle and lateral pins to the exiting nerve root was $3.2 \mathrm{~mm}, 1.1 \mathrm{~mm}$ and $-0.2 \mathrm{~mm}$ respectively (negative value denotes that the pin was lateral to the root). The pin pierced the nerve root on $2 / 16$ passes from the middle position and 3/16 from the lateral position. The overall average distance to the lumbar plexus was $14.3 \mathrm{~mm}$, $9.1 \mathrm{~mm}$ and $5.1 \mathrm{~mm}$ for the medial, middle and lateral trajectories, respectively.

Discussion/Conclusion: In regards to maintaining a safe distance from both the exiting nerve root as well as the lumbar plexus, the safest trajectory was the medial pin, passing just adjacent to the SAP. There was no incidence of nerve root injury with this trajectory however the close proximity means that neuromonitoring as well as tubular dilators would be necessary in the clinical setting.
RF Paper 14. High-Dose Parathyroid Hormone (1-34) Treatment Combined with Low-Dose rhBMP-2 in a Rabbit Model of Lumbar Spinal Fusion

Christina Holmes, $P h D^{l}$, Benjamin Elder, $M D, P h D^{2}$, Wataru

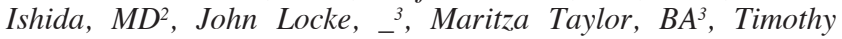
Witham, $M D^{\prime}$

${ }^{1}$ Johns Hopkins University School of Medicine, Baltimore, MD, ${ }^{2}$ Johns Hopkins University School of Medicine, Baltimore, Maryland, ${ }^{3}$, Baltimore, Maryland

Background/Introduction: Parathyroid hormone (1-34) (PTH) treatment has been shown to increase bone mass and reduce the risk of fracture in osteoporotic patients. Previously, we demonstrated that low-dose $(10 \mu \mathrm{g} / \mathrm{kg})$ PTH (1-34) treatment resulted in increased fusion mass volume in a rabbit model. However, this did not translate into improved biomechanical stiffness and did not improve fusion when combined with BMP-2 treatment (Lina et al, Spine [Phila Pa 1976]. 2014; 39(5):347). Studies in rodent models have shown that the effects of PTH (1-34) on spinal fusion may be dose-dependent (Ming et al, Spine [Phila. Pa. 1976]. 2012; 37(15): 1275; Abe et al, Bone.2007; 41(5): 775). We thus aim to evaluate whether higher doses of PTH (1-34) increase both the fusion mass volume and biomechanical stiffness and/or exhibits synergistic effects with low-dose BMP-2 treatment.

Materials/Methods: Posterolateral intertransverse process lumbar spinal fusion surgery was performed on 60 male, New Zealand White rabbits. Six experimental groups were evaluated: [1] iliac crest autograft alone $(\mathrm{n}=10)$; [2] autograft $+20 \mu \mathrm{g} / \mathrm{kg}$ PTH (1-34) (n = 10); [3] autograft $+40 \mu \mathrm{g} / \mathrm{kg}$ PTH(1-34) $(\mathrm{n}=$ 10); [4] rhBMP-2 alone ( $=10)$; [5] rhBMP-2 + $20 \mu \mathrm{g} / \mathrm{kg}$ PTH (1-34); and, [6] rhBMP-2 +40 $\mu \mathrm{g} / \mathrm{kg}$ PTH (1-34). Fusion was assessed 6 weeks post-operatively via manual palpation and CT analysis. Fusion mass mechanical stiffness was evaluated via fourpoint bending nondestructive biomechanical testing.

Results: In the autograft groups, $\mathrm{PTH}(1-34)$ treatment yielded increased rates of fusion via manual palpation, with increasing doses leading to increased fusion mass volume as assessed via CT. However, increased mechanical stiffness was only observed in the $20 \mu \mathrm{g} / \mathrm{kg}$ group via four-point bending tests.

Discussion/Conclusion: The observed increase in fusion mass mechanical stiffness in the $20 \mu \mathrm{g} / \mathrm{kg}$ PTH(1-34) group, but not the $40 \mu \mathrm{g} / \mathrm{kg}$ group, suggests that there may be an optimal dose in the rabbit model. We are currently studying groups treated with both PTH (1-34) and low-dose BMP-2.

RF Paper 15. Trisulfated-Glycosylated Peptide Amphiphile Nanofiber Scaffold for Spinal Arthrodesis

Joseph Weiner, $B S^{1}$, Danielle Chun, BA ${ }^{2}$, Ralph Cook, $B S^{2}$, Michael Schallmo, BS ${ }^{1}$, Sungsoo Lee, PhD ${ }^{3}$, Timmy Fyrner, PhD ${ }^{4}$, Mark McClendon, PhD ${ }^{3}$, Samuel Stupp, PhD , Wellington Hsu, M.D. ${ }^{6}$, Erin Hsu, PhD ${ }^{l}$

${ }^{1}$ Northwestern University Department of Orthopaedic Surgery, Chicago , IL , ${ }^{2}$ Northwestern University Department of Orthopaedic Surgery, Chicago, IL, ${ }^{3}$ Northwestern University Department of Materials Science and Engineering,, Chicago, IL, ${ }^{4}$ Northwestern University Department of Materials Science and Engineering, Chicago , IL, ${ }^{5}$ Northwestern University Department of Materials Science and Engineering, Chicago, IL, ${ }^{6}$ Northwestern Memorial Hospital, Chicago, Illinois

Background/Introduction: Use of recombinant human BMP-2 (rhBMP-2) has decreased the rate of pseudarthrosis 
after spine fusion procedures. However, reported complications have provoked considerable interest in the development of novel scaffolds, such as self-assembling nanofiber peptide amphiphiles (PA), that allow for reduced concentrations of rhBMP-2 without compromising its therapeutic effects. Our lab has shown that PAs synthesized with a trisulfated monosaccharide(TriS-GPA) mimic natural glycosaminoglycans (GAGs) and can bind to BMP-2. The aim of this study was to evaluate the efficacy of a trisulfatedGPA nanofiber scaffold in promoting spinal arthrodesis in a rat posterolateral fusion (PLF) model. We hypothesized that the TriSGPA scaffold will increase lumbar fusion rates relative to both a nonsulfated GPA and ACS scaffold with equivalently-loaded rhBMP-2.

Materials/Methods: 36 female Sprague-Dawley rats underwent L4-L5 PLF procedure with placement of one of three absorbable collagen sponge (ACS) scaffolds: (1) ACS +TriS-GPA, (2) ACS +non-sulfated GPA, or (3) ACS alone (negative control). All scaffolds were preloaded with $100 \mathrm{ng}$ rhBMP-2 per animal. ACS alone scaffolds were also pre-loaded with $10 \mu \mathrm{g}$ rhBMP-2 per animal as a positive control. Spines were evaluated for successful fusion and new bone formation via manual palpation, microCT imaging, and histologic analysis. Fusion scores were determined by blinded palpation using an established scoring system: $0=$ no bridging bone, $1=$ unilateral bridging, and $2=$ bilateral bridging bone. Spines with an average score of $\geq 1.0$ were considered fused.

Results: TriS-GPA preloaded with $100 \mathrm{ng}$ rhBMP-2,elicited significantly higher fusion scores relative to ACS and control GPA $(\mathrm{p}<0.001)$. The TriS-GPA preloaded with $0,10 \mathrm{ng}$, and $100 \mathrm{ng}$ rhBMP-2 elicited fusion rates of $0 \%, 8.3 \%$, and $100 \%$ respectively, which were also significantly higher than equivalently preloaded ACS (Figure 1).

Discussion/Conclusion: Our data suggests that PAs synthesized with a trisulfated monosaccharide(TriS-GPA) can effectively serve to bind BMP-2 within a fusion bed. Tris-GPA on an ACS carrier is capable of eliciting lumbar spine fusion while utilizing lower doses of rhBMP-2 than the established $100 \%$ fusion positive control model (10 $\mu \mathrm{g}$ rhBMP-2).

RF Paper 16. Comparison of Two Commercially available DBM against Autologous Bone in a Rat Posterolateral Spine Fusion Model

Samuel Grinberg, BA ${ }^{l}$, Antonio Brecevich, BA ${ }^{2}$, Paul Kiely, MD ${ }^{3}$, Byung Jo Yoon, M.S. ${ }^{4}$, Joseph Nguyen, MPH ${ }^{5}$, Frank Cammisa, $M D^{l}$, Celeste Abjornson, PhD ${ }^{l}$

${ }^{1}$ Hospital for Special Surgery, New York, New York, ${ }^{2}$ Hospital for Special Surgery, New York, New York, ${ }^{3}$ Nicklaus Children's Hospital, Miami, Florida, ${ }^{4}$ Hospitial for Special Surgery, New York, NY, ${ }^{5}$ Hospital for Special Surgery, New York, New York

Background/Introduction: Spinal fusion procedures are intended to stabilize the spinal column for a multitude of disorders. As an aid in the bone healing response, bone graft materials are utilized to bridge joints for arthrodesis and promote unions in pseudoarthrosis. Currently, the gold standard for stabilizing fusion masses in spinal procedures involves utilizing the osteogenic, osteoinductive, and osteoconductive properties of autologous iliac crest corticocancellous bone graft (ICBG). However, considerable morbidity is associated with harvesting the autologous graft. The objective of the study is to compare the quality and rate of fusion using a new generation of Demineralized Bone Matrix (DBM) against an established DBM and the 'gold standard' bone graft, ICBG, in the athymic rat posterior intertransverse process model.

Materials/Methods: A comparison of the quality and rate of fusion between DBM putty A (Accell Evo3®), Integra LifeSciences Corporation, Plainsboro, NJ) and DBM putty B (Grafton ${ }^{\circledR}$, BioHorizons ${ }^{\circledR}$, Birmingham, AL), with ICBG as the control, was performed. Fifty-one (51) athymic rats received a bilateral intertransverse process fusion, using DBM A, DBM B or ICBG. Fusions were evaluated using manual palpation, high resolution radiographic imaging, micro computed topography (micro-CT) and histological analysis. Twelve (12) rats (four from each group) were sacrificed at 3 weeks postoperatively, while the remaining thirty-six (36) were sacrificed at 8 weeks post-operatively.

Results: Manual palpation and nondestructive mechanical testing results indicated success of the test articles in forming a solid union. High-resolution radiography and micro-CT showed significant bone formation and bridging across the region between the transverse processes. Analysis of radiographic imaging highlights the superiority of fusion quality in the DBM treated rats compared to the ICBG treated rats. Radiographic grading according to the Lenke scale revealed $75 \%$ of rats in both DBM groups were graded as having large and trabeculated bilateral fusion masses compared to only $25 \%$ of the ICBG treated rats.

Discussion/Conclusion: Overall, specimens treated with either DBM demonstrated better bone formation and fusion consolidation than those treated with ICBG. Comprehensively, histological analysis revealed the main findings of the study and demonstrated that DBM A exhibited greater biologic activity at 8 weeks than DBM B.

\section{RF Paper 17 - Retracted}

RF Paper 18. Safety and Mode of Action Assessment of a Novel Progenitor Cell Therapy in a Gottingen Minipig Model for the Treatment of Degenerative Disc Disease

Lara Silverman, $P h D^{l}$, Galina Dulatova, $P h D^{2}$, Terry Tandeski, $P h D^{2}$; Kevin Foley, $\mathrm{MD}^{3}$

${ }^{1}$ University of Tennessee, DiscGenics, Salt Lake City, UT, ${ }^{2}$ DiscGenics, Salt Lake City, UT, ${ }^{3}$ Semmes-Murphey Neurologic Institute, University of Tennessee, DiscGenics, Salt Lake City, UT

Background/Introduction: Cell therapy is a promising approach to treating symptomatic degenerative disc disease, which currently has limited therapeutic options. We have developed a novel progenitor cell therapy derived from human disc tissue, which we term injectable discogenic cell therapy (IDCT). In this study, the allogeneic and xenogenic forms of the therapy were applied to Gottingen minipigs to assess safety and mode of action outcomes over 6 weeks post-treatment, as well as evaluate the duration of cellular persistence.

Materials/Methods: This IACUC-approved study utilized 15 Gottingen minipigs. Three discs per animal were injured using an optimized needle puncture method presented at LSRS 2015. Four weeks later, cohort 1 (6 animals) received one $150 \mu$ injection containing 100,000 human cells/disc of cells+vehicle, vehicle control, or sham ( $\mathrm{n}=6 /$ condition). Cohort 2 (9 animals) received human $(n=9)$ or pig $(n=18)$ IDCT doses loaded with nanocrystals (QTracker 625). Body weight and clinical observations were obtained. X-rays were taken at $0,4,6$ and 10 weeks to assess disc height index. Six weeks after treatment, cohort 1 spines were prepared for histology (H\&E, safO and Masson's trichrome). Cohort 2 spines were sacrificed at day 1 , week 2 and week 6 and the nanocrystals visualized using a confocal microscope. Statistical analysis: 1-way ANOVAs with post hoc tests $(\mathrm{p}<0.05)$.

Results: No safety concerns were noted in either xenograft or allograft treatments. IDCT-treated discs contained a centrally located region of collagen and proteoglycan consistent with intervertebral tissue. Disc height index decreased with injury and was significantly improved after treatment $(\mathrm{p}<0.05)$. Cells persisted to day 1 , but were not found by 2 or 6 weeks. 
Discussion/Conclusion: The large animal model provides support for the safety of IDCT. Additionally, this study demonstrated that within 2 weeks, the cells cause an increase in disc height with the deposition of collagen and proteoglycan in the central region of the disc. Cell disappearance was not due to xenograft rejection. Human clinical trials are anticipated.

RF Paper 19. In Vivo Mechanical Function and Remodeling of Disc-Like Angle Ply Structures (DAPS) for Total Disc Replacement

John Martin, PhD ${ }^{l}$; Sarah Gullbrand, PhD ${ }^{2}$, Dong Hwa Kim, $P h D^{I}$, Lachlan Smith, $P h D^{I}$, Dawn Elliott, PhD ${ }^{3}$, Robert Mauck, $P h D^{l}$, Harvey Smith, $M D^{l}$

\section{${ }^{1}$, Philadelphia, Pennsylvania, ${ }^{2}$, Philadelphia, PA, ${ }^{3}$, Newark , DE}

Background/Introduction: For the treatment of end-stage disc disease, total disc arthroplasty with a cellular, engineered replacement is a promising alternative to fusion; a viable, functional substitute may restore normal mechanics to the degenerated spine. To that end, our lab has created disc-like angle ply structures (DAPS) to mimic the structure and function of the native disc, combining an electrospun nanofibrous annulus fibrosus (AF) with a hydrogel nucleus pulposus (NP). Here, we report on the functional and compositional features of pre-matured DAPS over a period of 5 weeks of in vivo implantation.

Materials/Methods: DAPS were fabricated for the rat caudal disc space, seeded with bovine disc cells, and cultured for 5 weeks prior to in vivo implantation. Pre-matured DAPS were implanted into the $\mathrm{C} 8 \mathrm{-C}$ 9 rat caudal disc space, and stabilized with a custom ring-type external fixation device. At $1,3,7,17$, or 35 days $(n=4$ 6 per time point), vertebra-DAPS-vertebra motion segments were analyzed via MRI T2 mapping and alcian blue (proteoglycans) and picrosirius red (collagen) stained histology. Motion segments were mechanically tested in compression ( 20 cycles, 0 to $-3 \mathrm{~N} / \sim 0.3$ $\mathrm{MPa}$ ) after 5 weeks in vivo to quantify toe and linear region modulus, as well as transition and maximum strain.

Results: After 5 weeks in vivo, DAPS were mechanically functional, with toe and linear region compressive moduli matching that of the native disc [Fig 1a-b]. An elongated toe region and higher transition strain was present in DAPS. The NP T2 signal was super-physiologic at day 1 and progressively decreased over 5 weeks [Fig 1c]. MRI findings were reflected in histological results [Fig 1d]; NP composition was initially proteoglycan- and collagen-rich, but at 5 weeks post-implantation, composition was dominated by collagen.

Discussion/Conclusion: After 5 weeks of implantation in vivo, DAPS mechanical properties were similar to that of native tissue, indicating the potential of this engineered implant for functional restoration of motion segment mechanics. However, significant alterations in DAPS structure and composition occurred over this time period, with evidence of remodeling of the cell-seeded hyaluronic acid NP region. Future work will optimize preculture, implantation, and remobilization strategies to further improve implant performance in vivo.
RF Paper 20. Regeneration of the Intervertebral Disc: Evidence Using a Defined Molecular Approach Based Upon the Notochordal Cell Secretome

AjayMatta, $P_{h} D^{1}$, Muhammad Karim,DVMMSC MPhil(Hons) $)^{2}$; William Erwin, $D C P h D^{3}$, David Isenman, $P h D^{4}$

${ }^{1} \mathrm{krembil}$ research institute, Toronto, Ontario, ${ }^{2} \mathrm{Krembil}$ Research Institute, Toronto, Ontario, ${ }^{3}$ Krembil Research Institute/Toronto Western Hospital, Toronto, Ontario, ${ }^{4}$ University of Toronto, Toronto, Ontario

Background/Introduction: We have previously reported that notochordal cell conditioned medium (NCCM)can suppress the catabolic effects of inflammatory cytokines upon nucleus pulposus cells as well stimulate anabolic activity and suppress apoptosis stimulating. Furthermore, we have demonstrated that a single injection of NCCM into the degenerative disc in a pre-clinical rodent model of degenerative disc disease (DDD) can protect the IVD from degeneration. Here we have identified and characterized the necessary and sufficient factors secreted by notochordal cells that can rescue the degenerative disc.

Materials/Methods: We developed NCCM following our established methods and after filtration and centrifugation we subjected the NCCM to FPLC column chromatography from which we obtained 16 molecular weight specific fractions. These fractions were then bioassayed using bovine caudal discs in vitro in the presence of a chemotherapeutic agent 'etoposide' and were assayed for activated caspase $-3 /-7$ activity at 24 and 48 hours. Three fractions demonstrated suppressed caspase $-3 /-7$ activity as well as increased cell viability (MTT assay). LC-MS/MS mass spectroscopy was then used to identify the proteins within these 'active' fractions producing a list of 377 non-redundant proteins. After subsequent in vitro optimization we injected a total volume of $8 \mathrm{ul}$ containing TGFB1+CTGF (factors identified from within the active fractions) into a degenerative rat-tail model of DDD (developed within our lab) and PBS into control discs. Eight weeks post injection we assayed the effects of the active factor injection compared to control PBS injections (12-weeks post injection) using Western blotting and immunohistochemical methods.

Results: A single injection of TGFB1+CTGF induced repair/ regeneration of the degenerative disc including restoration of stem cell and notochordal cell markers and suppressed inflammation whereas PBS injections did not and the control injections resulted in a degenerative, fibrocartilagenous IVD NP. Furthermore, the presence of TGFB, CTGF and CCN5 (WISP2) are undetectable in human, chondrodystrophic dog and degenerative rat-tail discs indicating the importance of this signaling pathway in the development of DDD.

Discussion/Conclusion: Here, for the first time we reveal the necessary and sufficient factors secreted by notochordal cells that are capable of restoring/regenerating DDD induced in a preclinical animal model that could serve as novel, molecular therapy for DDD.

RF Paper 21. Lumbopelvic Fixation Provides Better Fixation Than One Trans-Sacral Screw in Complex Sacral Fractures

Ehsan Jazini, $M D^{l}$, Noelle Klocke, $M S^{2}$, Oliver Tannous, $M D^{l}$, Tristan Weir, BS ${ }^{1}$, Herman Johal, $M D, P h D^{l}$, Daniel Gelb, $M D^{\prime}$, Jascone Nascone, $M D^{l}$, Robert O'Toole, $M d^{1}$, Brandon Bucklen, $P h D^{2}$, Steven Ludwig, $M D^{l}$

${ }^{1}$, Baltimore, MD, ${ }^{2}$, Audubon, PA

Background/Introduction: To determine whether biomechanical stability is greater when lumbopelvic fixation (LPF) 
is used to treat both small $(1 \mathrm{~mm})$ and large $(1 \mathrm{~cm})$ comminutions within zone II, class C-type sacral fractures when anatomical variability and fracture severity traditionally dictates use of a single trans-sacral screw fixation.

Materials/Methods: Flexion-extension was simulated using a "floating hip model" (L4-Pelvis: $\mathrm{n}=7$ ). Pure bending moments $(\leq 8 \mathrm{Nm})$ were applied at L4; the right hemipelvis was permitted 5 degrees-of-freedom and the left was fully constrained. Two surgical techniques were applied: LPF with bilateral cannulated iliac screws (L4-P) and pedicle screws at L4 and L5 connected via a cross-connector; and traditional fixation (T1) with a single trans-sacral screw. Both also had anterior pubis symphysis plates. Two comminution severities were simulated through the sacral foramen: 1) single vertical fracture (small gap, $1 \mathrm{~mm}$ ); or, 2) two vertical fractures, $10 \mathrm{~mm}$ apart with intermediary bone removed (large gap). Relative Eulerian motion was assessed between iliac crests for the pelvic ring (PRM) and across the intended sacral fracture zone (SFM). Motions were normalized to average intact range of motion, and two-way ANOVA revealed significant differences $(\mathrm{p}<0.05)$ and effects associated with fracture size, surgical technique, and interaction effects of the two. A student t-test $(\mathrm{p}<0.05)$ assessed differences between techniques within a given fracture size (LPF vs T1).

Results: The large gap model SFM was significantly reduced ( $\mathrm{p}<0.05$, t-test) with L4-P $(76.4 \pm 22.5 \%)$ versus T1 $(193.9 \pm 25.0 \%)$, but not in the small gap model (L4-P=90.2\% $\pm 85.6 \%$ vs. $\mathrm{T} 1=61.9 \pm 45.8 \%$, Figure). PRM was not significantly different between the two constructs in either the small or large gap models. Two way ANOVA tests revealed that increasing the fracture gap size significantly increased the SFM, regardless of surgical treatment; yet gap size had a greater effect when a traditional construct was used $(\mathrm{T} 1, \mathrm{p}<0.05)$. PRM was not significantly affected by gap size or fixation technique.

Discussion/Conclusion: When two trans-sacral fractures are not clinically feasible, LPF provides significantly increased stability across the sacral fracture over placement of a single trans-sacral screw. The significant negative consequences of fracture gap size on trans-sacral screw fixation biomechanics for comminuted complex sacral fracture makes LPF important for a traumatologist's armamentarium.

RF Paper 22. Percutaneous Lumbopelvic Fixation Technique for Reduction and Stabilization of Sacral Fractures with Spinopelvic Dissociation Patterns

\section{Seth Williams, $M D$}

\section{University of Wisconsin , Madison, WI}

Background/Introduction: Sacral fractures with spino-pelvic dissociation patterns are highly unstable injuries. Reestablishing the anatomic relationship of the pelvis to the axial spine and achieving mechanical stability, while preserving neurological function, are the main treatment goals. Lumbopelvic fixation is tyipically performed through a single posterior extensile midline incision from L4 to the pelvis. Surgery can be lengthy with major blood loss, and associated with wound complications. In an effort to minimize the morbidity of surgery while achieving stable fixation, we developed a percutaneous lumbopelvic reduction and fixation technique.

Materials/Methods: Between March 2009 and April 2015, 17 consecutive patients with sacral fractures causing spino-pelvic dissociation were treated with percutaneous lumbopelvic fixation using lumbar pedicle screws connected to iliac screws with rods. Surgery was performed through separate paired parasagittal incisions under fluoroscopic guidance. Data were collected prospectively including blood loss, fluoroscopy time, screw accuracy, wound complications, neurological status, cases of instrumentation failure, fracture healing, and patient functional status.

Results: Surgical duration averaged 2 hours 24 minutes and blood loss averaged $202 \mathrm{~mL}$. There were no cases of instrumentation failure. Reduction was attempted in 6 patients and was felt to be successful in 4 patients, with an average preoperative kyphosis of 16 degrees and an average post-operative kyphosis of 4 degrees. A traumatic sacral spondyloptosis was anatomically reduced. There was one deep wound infection. All fractures healed with an average follow up of 22 months. All but two patients regained normal bladder function.

Discussion/Conclusion: Sacral fractures with spinopelvic dissociation patterns can be reduced and stabilized with percutaneous lumbopelvic instrumentation. Fracture reduction was not successful in all cases, but became more reliable as our technique evolved. This technique can be performed safely with limited blood loss and within a reasonable surgical duration, theoretically minimizing the physiological burden of surgery in these severely injured patients.

RF Paper 23. A Posterior-Only Lumbopelvic Approach Is Not Biomechanically Stable Enough for a Complex Sacral Zone II Fracture With Anterior Pubis Symphysis Disruption

Ehsan Jazini, $M D^{l}$, Noelle Klocke, $M S^{2}$, Oliver Tannous, $M D^{l}$, Tristan Weir, BS $S^{I}$, Herman Johal, $M D, P h D^{I}$, Daniel Gelb, $M D^{l}$, Jascone Nascone, $\mathrm{MD}^{l}$, Robert O'Toole, $\mathrm{Md}^{l}$, Brandon Bucklen, $P h D^{2}$, Steven Ludwig, $M D^{l}$

\section{${ }^{1}$, Baltimore, $\mathrm{MD},{ }^{2}$, Audubon, PA}

Background/Introduction: To determine whether an anterior pubis symphysis plate is biomechanically necessary to provide full pelvic ring stabilization when lumbopelvic fixation is used to treat zone II sacral fractures with anterior symphysis disruption, or if a posterior-only approach can be used in lieu of a $360^{\circ}$ procedure.

Materials/Methods: A "floating hip model" was used to simulate bilateral stance during flexion-extension moments $(\leq 8 \mathrm{Nm})$ applied to fresh-frozen cadaveric lumbopelvic specimens, which had been previously cleaned and potted at L4 and at the left ischium (Figure). Following intact motion collection, the pubis symphysis was transected, and a small gap fracture model was simulated $(<1 \mathrm{~mm})$ by creating a vertical fracture through the sacral foramen. Next, bilateral cannulated iliac screws were placed in the pelvic wings, and rods (with cross-connectors) connected the pedicle screws placed in L4 and L5. The motion profile was then captured both with (L4-P+Ant: Small Gap) and without (L4P: Small Gap) an anterior pubis symphysis plate $(n=7)$. A larger fracture gap model $(1 \mathrm{~cm})$ was created along the previously created fracture line. Use of an anterior plate was also tested (L4-P+Ant: Large Gap and L4-P: Large Gap, $n=4)$. Sacral fracture zone motion (SFM), pelvic ring motion (PRM), and total lumbopelvic motion (LPM) were normalized on a per-specimen basis, and were evaluated with student t-tests $(\mathrm{p}<0.05)$.

Results: Average motion was always greater than that observed within intact, regardless of anterior plate use, across SFM and PRM (Figure). The anterior plate always reduced overall motion; the only instance of statistical significance was found within the SFM of the small gap model, although findings approached significance $(p=0.078)$ within the SFM large gap model.

Discussion/Conclusion: Use of an anterior plate leads to reduced motion when the pubis symphysis is disrupted in conjunction with zone II sacral fractures of varying complexities. Despite non-significant findings, standard deviations of motion were high when the anterior plate was not added to the surgical construct. This demonstrates that permissible motion was much 
more variable and in some instances substantially greater with this construct. These findings suggest that a $360^{\circ}$ approach may be necessary with such complex traumatic injuries.

\section{RF Paper 24. Lumbosacral Epidural Lipomatosis: a Matched Control Study}

Varun Puvanesarajah, $B S^{1}$, Baris Yildirim, $B S^{2}$, Howard An, $M D^{3}$, Amit Jain, $M D^{4}$, Francis Shen, $M D^{2}$, Hamid Hassanzadeh, $M D^{1}$

${ }^{1}$, Charlottesville, VA, ${ }^{2}$, Charlottesville, Virginia, ${ }^{3}$ Rush University Medical Center, Chicago, IL , ${ }^{4}$, Baltimore, MD

Background/Introduction: First described in 1975, epidural lipomatosis is hypertrophy of adipose tissue in the epidural space often diagnosed on magnetic resonance imaging (MRI). We present the largest known series of epidural lipomatosis patients, with over 200 consecutive cases, to characterize demographics and clinical symptoms of patients who demonstrated epidural lipomatosis on MRI, and to compare these characteristics against a matched control group.

Materials/Methods: Patients evaluated for pathology requiring lumbar MRI imaging between September 2010 and September 2015 were retrospectively reviewed and were included in this study if lumbosacral epidural lipomatosis (Figure 1) was listed as a diagnosis on a radiological note during any visit to our academic medical center. We identified 230 patients who fulfilled the study criteria and were included in the final analysis. Follow-up time for the cohort was $24.9 \pm 19.0$ months (range 1.2 to 67.9 months). A separate patient cohort of 230 unique, age- and gender-matched controls without lumbosacral epidural lipomatosis was created from a database of patients who had lumbar MRI imaging during the same study period.

Results: Average age at diagnosis was $55.0 \pm 12.6$ years (range 25 to 88 years). One hundred and fifty-three patients $(66.5 \%)$ were male. Patients with epidural lipomatosis were more likely to have history of diabetes mellitus type 2 (OR 2.20,95\% CI 1.43 to 3.39 ), history of prior statin use (OR $1.86,95 \%$ CI 1.25 to 2.78 ), or be on disability (OR 2.72, 95\% CI 1.72 to 4.30). Furthermore, patients with epidural lipomatosis had a significantly increased BMI compared to controls (37.6 vs $30.5 \mathrm{~kg} / \mathrm{m} 2$, p < 0.001 ).

Discussion/Conclusion: Patients with lumbosacral epidural lipomatosis tend to be obese with high incidences of diabetes mellitus type 2 and history of statin use. Further studies are required to understand the pathogenesis, risk factors, and treatment outcomes of patients with epidural lipomatosis.

RF Paper 25. Quantifying the Learning Curve for Minimally Invasive Transforaminal Lumbar Interbody Fusion

Abhishek Kumar, MD, FRCSC ${ }^{1}$, Robert Merrill, BS ${ }^{2}$, Joshua Meaike, $B S^{3}$, Samuel Overley, $M D^{3}$, Dante Leven, DO ${ }^{4}$, Sheeraz Qureshi, $M D, M B A^{2}$

${ }^{1}$ Mount Sinai Medical Center, New York , NY, ${ }^{2}$ Mount Sinai Medical Center, New York, NY, ${ }^{3}$, New York, NY, ${ }^{4}$, NY, NY

Background/Introduction: Minimally invasive transforaminal lumbar interbody fusion (MI-TLIF) has been demonstrated to carry benefits over open TLIF in terms of intraoperative morbidity, postoperative recovery and return to function. However, preconceptions regarding the learning curve have lead to hesitancy in adopting this novel technique. Our goal was to quantify the learning curve by tabulating operative statistics over the first 5 years of a single surgeon's experience with MI-TLIF.
Materials/Methods: Data for all single level, primary MI-TLIF performed between 2011 and 2015 were collected from the hospital electronic medical record and PACS system. An analysis for statistical correlation was performed comparing each operative endpoint with chronologic case number. For each endpoint that had a statistically significant correlation we identified the learning curve by pinpointing the case number after which the correlation between the endpoint and case number was no longer significant $(p=>0.05)$. This identifies the time point after which further experience does not confer additional benefit. Complications included any event that led to either readmission to hospital within 30 days of discharge or re-operation at any time point.

Results: We analyzed a total of 111 patients including 57 males and 54 females. Overall, an MI-TLIF required $121.7 \pm 38.3$ minutes of operative time with an estimated blood loss (EBL) of $65.6 \pm 48.0 \mathrm{ml}, 152.5 \pm 52.9$ seconds of fluoroscopy time and a $1.79 \pm 1.38$ day hospital admission. The overall complication rate was $14.6 \%$. Learning curve with respect to operative time, fluoroscopy time and complication rate was identified at case 17, 40 and 47 respectively. In terms of EBL and length of stay, there was no evidence of a correlation with case number.

Discussion/Conclusion: In chronicling one surgeon's experience with MI-TLIF we find that technical proficiency in terms of performing the surgery can be reasonably achieved after 15 to 20 cases. Mastery of the technique, evidenced by a lower complication rate and shorter fluoroscopy time, may require 40 to 50 cases. Surgeons looking to add this technique to their repertoire should be cognizant of these findings and seek appropriate training.

RF Paper 26. X-ray Reliability to Detect Spinous Process Fractures after Placement of Interspinous Process Spacer

William Lavelle, $M D^{I}$, Aaron Bianco, $M D^{l}$, Mark Palumbo, $M D^{2}$, Ian Madom, $M D^{l}$, Richard Tallarico, $M D^{l}$, Mauricio Valdes, $\mathrm{MD}^{3}$, Michael Galgano, $\mathrm{MD}^{4}$; Henaku Yirenkyi, $\mathrm{MD}^{4}$, Fred Geisler, $M D, P h D^{5}$

${ }^{1}$, Syracuse, NY, ${ }^{2}$ Department of Orthopaedics, Division of Spine Surgery, Warren Alpert Medical School of Brown University , Providence, RI, ${ }^{3}$ Hedley Orthopaedic Institute, Phoenix, AZ, ${ }^{4}$ SUNY Upstate Medical University, Syracuse, NY, ${ }^{5}$ Rhausler, Inc., San Carlos, CA

Background/Introduction: Interspinous devices are a potentially less invasive method to manage moderate lumbar stenosis. Spinous process fractures after placement of interspinous spacer devices are documented at $22 \%$ and with spondylolisthesis $52 \%$ raising questions about the ability to recognize fractures on standard post procedural imaging. Our study examined the reliability and accuracy of orthopedic spine surgeons to identify spinous process fractures on plain radiographs.

Materials/Methods: This was a prospective, multicenter IDE study comparing Superion to non-inferior X-stop. Surgeons submitted postoperative plain radiographs to the sponsor. Medical Metrics completed independent reviews of imaging by radiologists confirming fractures through CT scans. Radiographic confirmation of at least moderate spinal stenosis was required. Patients placed into four groups: Superion devices with and without postoperative fractures and X-stop devices with and without postoperative fractures. Plain radiographs were de-identified and randomized. Five orthopedic spine surgeons reviewed films and identified if postoperative spinous process fracture was present. Two subjects from each group presented again allowing collection of intrareliability. Surgeons' accuracy compared to the radiologists.

Results: Overall single measure reliability for surgeons reviewing the image was 0.341 . Average measures reliability was 0.721 . Overall intra-observer reliability was 0.632 for a 
single measure method and 0.760 for an average measures method. Overall accuracy with respect to reported fracture by the radiologist confirmed by a CT scan was $69.6 \%$.

Discussion/Conclusion: Initial IDE studies for X-stop device reported a fracture rate of $1 \%$ at two years. Conversely, studies recently completed identified much higher rates of postoperative spinous process fractures of $28.9 \%$. High rates of spinous process fractures identified on CT compared to x-ray raises the question of needing routine postoperative CT scans. Our study demonstrated only fair to moderate inter-observer reliability while having good intra-observer reliability on plain radiographs. We had $70 \%$ accuracy identifying spinous process fractures. A limitation to this study was the possibility other patients had fractures missed by radiologists. Spinous process fractures are likely under reported on plain radiograph films. Our data demonstrates concern for the accuracy and reliability of postoperative plain x-ray to identify spinous process fractures after placement of interspinous process devices.

RF Paper 27. Does Level of Response to SI Joint Block Predict response to SI Joint Fusion?

David Polly, Jr, MD ${ }^{1}$; Jonathan Sembrano, $\mathrm{MD}^{2}$, Charles Ledonio, $M D^{3}$, Daniel Cher, $M D^{4}$

${ }^{1}$ University of Minnesota, Minneapolis, MN, ${ }^{2}$, Minneapolis , MN, ${ }^{3}$, Minneapolis, MN, ${ }^{4}$ SI - Bone, Inc., San Jose, CA

Background/Introduction: Threshold percent pain relief required to diagnose sacroiliac joint (SIJ) dysfunction during a diagnostic SIJ block (SIJB) is not known. Although there is no gold standard, one reference standard is response to definitive treatment.

Materials/Methods: Subgroup analysis of 320 subjects in two prospective trials of SIJ fusion: INSITE (NCT01681004) a randomized trial vs. nonsurgical management (NSM) and SIFI (NCT01640353), a singlearm study. All participants were diagnosed with SIJ dysfunction via history, at least 3 positive provocative physical examination signs, and a standardized diagnostic SIJB using fluoroscopically guided anesthetic injection to the target joint with a reduction of at least $50 \%$ in pain at 30 or 60 minutes after SIJB. The degree of improvement in VAS SIJ pain and Oswestry Disability Index (ODI) scores, expressed as absolute or percentage improvement from baseline, was correlated with the average acute improvement in SIJ pain during the SIJB, calculated as per Cohen et al.

Results: Average pain reduction during the first hour after SIJB was $79 \%$. This was unrelated to standard demographic predictors. Six months after SIJ fusion, the mean VAS SIJ pain reduction was 50.9 (28.6) points and the mean ODI reduction was 24.6 (20.5) points. SIJ pain and ODI reductions at 12 months in the SIJ fusion group were 50.8 (29.2) and 25.8 (20.5) points, respectively. There was little correlation in 6 or 12 month improvements in SIJ pain or ODI with average reduction in pain during SIJB. In the SIJF group, the proportion of subjects achieving meaningful reductions in SIJ pain and ODI scores did not vary by average response during SIJB ( $\mathrm{p}=0.8407$ and 0.3069 and $\mathrm{p}=0.6368$ and 0.5913 ). Similarly, response during SIJB did not predict the proportion of patients with followup pain scores $<30$ or $\mathrm{ODI}<20$.

Discussion/Conclusion: The degree of improvement in acute pain during an SIJB did not predict 6month improvements in VAS SIJ pain or ODI score. $50 \%$ acute pain reduction threshold during SIJB resulted in excellent surgical outcomes. The use of overly stringent selection criteria for determining which patients should undergo SIJF may serve to withhold a beneficial procedure from a substantial number of patients with SIJ dysfunction.
RF Paper 28. Narcotic Consumption Following a Minimally Invasive Transforaminal Lumbar Interbody Fusion: An Age Based Analysis

Dustin Massel, $B S^{l}$; Benjamin Mayo, BA ${ }^{l}$, William Long, $B A^{l}$, Krishna Modi, $B S^{l}$, Daniel Bohl, $M D, M P H^{2}$, Kern Singh, $M D^{l}$

${ }^{1}$ Rush University Medical Center, Chicago, IL, ${ }^{2}$ Rush University Medical Center, New Haven, CT

Background/Introduction: Elderly patients are at a greater risk of narcotic related adverse events and are may benefit from techniques to minimize postoperative narcotic consumption. The purpose of this study is to examine postoperative narcotic consumption based on patient age following a minimally invasive (MIS) transforaminal lumbar interbody fusion (TLIF).

Materials/Methods: A prospectively maintained database of patients who underwent a primary single-level MIS TLIF between 2007-2015 was reviewed. Patients were stratified into four cohorts by age: $<40,40-49,50-59$, and $\geq 60$ years. Differences in patient demographics and preoperative characteristics were assessed using independent sample t-tests and Chi-square analysis. Peri- and postoperative outcomes were analyzed using Poisson regression with robust error variance (binary outcomes) or linear regression (continuous outcomes) adjusted for patient demographics, preoperative characteristics, and perioperative variables.

Results: A total of 383 patients were identified, of which 74 $(19.3 \%)$ were $<40,90(23.5 \%)$ were $40-49,108(28.2 \%)$ were $50-59$, and $111(29.0 \%)$ were $\geq 60$ years old. The older cohorts had higher percentages of females $(p<0.001)$, non-smokers $(p=0.007)$, operations at the L4-L5 level ( $\mathrm{p}<0.001)$, and a greater comorbidity burden $(p<0.001)$ compared to younger cohorts. The oldest cohort demonstrated the lowest preoperative VAS score $(p=0.013)$, but no trend across cohorts was observed. Increasing age was associated with reduced narcotic utilization on postoperative days (POD) 1, 2, 3 and in total (POD 1: $\mathrm{p}=0.028$; POD 2: $\mathrm{p}=0.001$; POD 3: $\mathrm{p}=0.024$; Total: $\mathrm{p}=0.001$ ). Older age was associated with greater improvements in pain at 6 weeks postoperatively $(\mathrm{p}=0.001)$ and a higher, but not statistically significant, arthrodesis rate $(\mathrm{p}=0.076)$. The 40-59 years old cohorts experienced greater complication rates than those $>60$ or $<40$ years $(\mathrm{p}=0.031)$ (Table 2$)$.

Discussion/Conclusion: This analysis demonstrated distinct narcotic consumption patterns between age groups following a MIS TLIF. Patients $\geq 60$ years old had lower inpatient narcotic utilization compared to the other age groups, while patients $<40$ required the highest dosing. The results of this study suggest more conservative postoperative pain management plans may be reduce narcotic related side effects for older patients ( $\geq 60$ years old). Conversely, younger patients $(<40$ years old) may require greater narcotic dosing and care must be taken to avoid toxicity.

RF Paper 29. Risk Factors for Prolonged Post Operative Narcotic Use After Spine Surgery

Linda Suleiman, $M D^{l}$; Brett Rosenthal, $M D^{l}$, Abhishek Kannan, B.S. ${ }^{2}$, Adam Edelstein, MD ${ }^{I}$, Wellington Hsu, M.D. ${ }^{3}$, Alpesh Patel, $M D, F A C S^{\prime}$

${ }^{1}$ Northwestern University, Chicago, IL, ${ }^{2}$ Northwestern Feinberg School of Medicine, Chicago, Illinois, ${ }^{3}$ Northwestern Memorial Hospital, Chicago, Illinois

Background/Introduction: Patients undergoing elective spine surgery are at increased risk of opioid dependence. While opiates play an important role in perioperative pain control, prolonged use may indicate a failure to achieve goals of surgery. Risk factors for 
prolonged postoperative narcotic use after spine surgery are not well reported.

Materials/Methods: We queried the Illinois Prescription Monitoring Program (PMP) to investigate the use of Schedule II-V controlled substances by patients who underwent surgery by a single spine surgeon over a 5-year period. Prescription data from 3 and 6-month pre- and post-operative time points were obtained. Patients who received narcotics from outside the spine service were categorized as having multiple providers. All narcotic prescription dosages were converted to morphine equivalents for comparison. Demographic characteristics, psychiatric history, smoking status, alcohol use, BMI, type of procedure and 30-day outcomes were included in a multivariate regression model to determine risk factors for prolonged post-operative narcotic use.

Results: 264 patients met our inclusion criteria. 127 patients had 3-month preoperative narcotic use, $92(72.4 \%)$ and 33 $(26 \%)$ of which had 3 and 6-month postoperative narcotic use, respectively. 55 patients with 6-month preoperative narcotic use, $44(80 \%)$ and $22(40 \%)$ of which had 3 and 6-month postoperative narcotic use, respectively. Patients with multiple prescribers 3 months preoperatively used significantly higher narcotics dosages $(\mathrm{p}=0.0011)$. Patients with 3 and 6 -month preoperative narcotic use had significantly higher 6-month postoperative narcotic use ( $\mathrm{p}=0.0034$ and 0.001 , respectively). Multivariate regression modeling showed 6-month preoperative narcotic use to be associated with increased risk of 6-month post-operative narcotic use ( $R R=2.96, C I=1.60-5.47)$, however other factors including age, gender, psychiatric history, smoking, alcohol use, marriage, BMI and surgical approach were not associated with increased rates of postoperative narcotic use (Table 1).

Discussion/Conclusion: Using a state-run controlled substance monitoring program, we have identified 6-month preoperative opioid use as the most significant predictor of prolonged narcotic use after spinal surgery. Preoperatively, patients with multiple prescribers had higher doses of narcotic dispensed to them. These findings will help surgeons preoperatively set patient expectations and predict clinical response to surgical management.

RF Paper 30. Iatrogenic Spondylolisthesis Following Laminectomy for Degenerative Lumbar Stenosis: Systematic Review and Current Concepts

Daipayan Guha, $M D^{1}$, Robert Heary, $M D^{2}$, Mohammed Shamji, $M D, P h D, F R C S C^{1}$

${ }^{1}$ University of Toronto, Toronto, ON, ${ }^{2}$ Rutgers, New Jersey Medical School, Newark, New Jersey

Background/Introduction: Decompression without fusion for degenerative lumbar stenosis is an effective treatment for both the pain and disability of neurogenic claudication. Iatrogenic instability following decompression may require further intervention to stabilize the spine. The authors review the incidence of postsurgical instability following lumbar decompression, and assess the impact of surgical technique as well as study design on the incidence of instability.

Materials/Methods: A comprehensive literature search was performed to identify surgical cohorts of patients with degenerative lumbar stenosis, with and without preexisting spondylolisthesis, who were treated with laminectomy or minimally-invasive decompression without fusion. Data on patient characteristics, surgical indications and techniques, clinical and radiographic outcomes, and reoperation rates were collected and analyzed.

Results: A systematic review of 24 studies involving 2496 patients was performed, assessing both open laminectomy and minimally-invasive bilateral canal enlargement. Postoperative pain and functional outcomes were similar across the various studies, and postoperative radiographic instability was seen in $5.5 \%$ of patients. Instability was seen more frequently in patients with preexisting spondylolisthesis $(12.6 \%)$ and in those treated with open laminectomy (12\%). Reoperation for instability was required in $1.8 \%$ of all patients, and was higher for patients with preoperative spondylolisthesis $(9.3 \%)$ and for those treated with open laminectomy $(4.1 \%)$.

Discussion/Conclusion: Instability following lumbar decompression is a common occurrence. This is particularly true if decompression alone is selected as a surgical approach in patients with established spondylolisthesis. This complication may occur less commonly with the use of minimally-invasive techniques; however, larger prospective cohort studies are necessary to more thoroughly explore these findings.

Poster 1. Cardiopulmonary complications in elective spine surgery

Peter Passias, $M D^{l}$, Gregory Poorman, BA ${ }^{2}$, Cyrus Jalai, $B A^{l}$

${ }^{1}$, New York, NY, ${ }^{2}$, Bronxville, NY

Background/Introduction: Cardiopulmonary complications are the leading causes of intra-operative mortality among elective spine patients. Understanding the risks for and predictors of these sentinel events is important for risk evaluation, and patient counseling.

Materials/Methods: This study was a retrospective review of elective (cervical, thoracolubmar) spine surgical patients from the American College of Surgeon's National Surgical Quality Improvement Program (NSQIP). Incidence of myocardial infarction, cardiac arrest, unplanned reintubation, on-ventilator $>48$ hours, peri-operative pneumonia, and pulmonary embolism intraoperative complications were estimated. Surgical characteristics were evaluated using CPT codes specific to spinal fusion and decompression. Multivariate analysis, controlling for Charlson comorbidity index (CCI), body mass index (BMI), race, sex, ASA class, smoking status, history of heart problems, ventilation dependence, pre-op blood transfusion, bleeding disorders, and fusions greater than 4 levels, evaluated the risk of intra-operative cardiopulmonary complications.

Results: 60,964 patients were identified. Cardiopulmonary incidence rates per 1,000 elective spine patients were 2.1 myocardial infarctions, 1.3 cardiac arrests, 4.3 unplanned intubations, 3.5 on-ventilator $>48$ hours, 6.1 peri-operative pneumonia, and 3.7 pulmonary embolisms. The strongest surgical procedural predictors for cardiopulmonary complications were thoracic approach (OR 3.0, CI 1.3-4.8, p<0.001) Smith-Petersen Osteotomy (OR 2.7, CI $2.0-3.8, \mathrm{p}<0.001$ ), and three-column osteotomy (OR 2.5, CI 1.3-4.8, $\mathrm{p}=0.007)$. The strongest demographic predictors for cardiopulmonary complications were $>10 \%$ weight loss within 6 months (OR 3, CI 1.7-5.2, p<0.001), pre-operative blood transfusion within 72 hours (OR 2.1, CI 1.0-4.1, p=0.057), and hemi/quadra/paraplegia (OR 2.0, CI 1.2-3.3, p=0.006). Patients with cardiac arrest had the highest mortality rate $(34.57 \%)$, followed by ventilator $>48$ hours $(11.74 \%)$, and unplanned reintubation (10.26\%). Patients on ventilators $>48$ hours increased the length of hospital stay the most (17.58 days), followed by unplanned reintubation (13.04), and peri-operative pneumonia (10.58).

Discussion/Conclusion: Presenting comorbidities and surgical invasiveness parameters were equally valuable in predicting for cardiopulmonary complications, as demonstrated by similar OR's. Included is a breakdown of demographic and surgical characteristics predicting cardiopulmonary events, and the complications' impact on peri-operative outcomes. 
Poster 2. The threshold of obesity as a comorbidity in the lumbar spine

\section{Peter Passias, $M D^{l}$, Gregory Poorman, $B A^{2}$, Cyrus Jalai, $B A^{l}$}

${ }^{1}$, New York, NY, ${ }^{2}$, bronxville, ny

Background/Introduction: Obesity is an established predictor for developing back problems and increases risk of complications during surgery. This paper investigates whether there is a BMI threshold in the lumbar spine at which obesity becomes a risk factor for complications, and profiles the increased morbidity of obese patients.

Materials/Methods: This study is a retrospective review of the American College of Surgeon's National Surgery Quality Improvement Program (NSQIP) nationwide database for patients with lumbar pathology. Obesity categories - underweight (BMI $<18.5)$, normal weight (20-29.9), and obesity class 1 (30-34.9), 2 (35-39.9), and $3(40+)$ - facilitated analysis of increasing BMI as a risk factor for different complications by category (cardiopulmonary, infection, airway, surgical, medical, and 'all'), as well as individually. Surgical invasiveness separated higher and lower overall-risk surgeries. Statistical analyses involved group ANOVA comparisons for complication rates between obesity groups, and multivariate analyses to identify changes in risk by obesity-status, controlling for CCI, smoking, race, sex, ASA classification, and surgical invasiveness.

Results: 47,586 lumbar spine patients were analyzed. Increases in BMI were associated with increases complication rates. Obesity had a statistically significant effect on complications compared to non-obese patients at $\mathrm{BMI}=35(\mathrm{p}=0.014)$, however class 1 and class 2 were statistically similar $(\mathrm{p}=0.423)$. There was a significant difference in complication rates between obesity classes 2 and 3 $(\mathrm{p}<0.001)$. In less-invasive surgeries, obesity by class, had a strong impact on renal insufficiency (class $1: \mathrm{OR}=3.1$, class 2: $\mathrm{OR}=3.7$, class 3: $\mathrm{OR}=3.7, \mathrm{p}<0.05$ ), and renal failure (class 3: $\mathrm{OR}=5.4$, $\mathrm{p}=0.005)$ as well as overall complication rates. In more invasive surgeries, obesity was associated with increased odds of sustaining a superficial infection (class $1: \mathrm{OR}=2.0$, class $2: \mathrm{OR}=2.5$, class 3 : $\mathrm{OR}=4.4, \mathrm{p}<0.005$ ) or a surgical complication (class 2 : $\mathrm{OR}=1.8$, class 3: $\mathrm{OR}=2.9, \mathrm{p}<0.05)$.

Discussion/Conclusion: Obesity has a similar effect on moreand less-invasive surgeries. Obesity has significant jumps in complication rates at BMI of 35 and 40 .

Poster 3. Intradiscal Pressure Changes in a Growing Rod Cadaver Model

Sarah Galvis, B.S. ${ }^{1}$, Joshua Arnold, B.S. ${ }^{1}$, Erin Mannen, Ph.D. ${ }^{l}$, Benjamin Wong, BASc ${ }^{l}$, Hadley Sis, B.S.', Eileen Cadel, B.S.', Dennis Anderson, Ph.D. ${ }^{2}$; Paul Arnold, $M D^{3}$, Lisa Friis, Ph.D. ${ }^{l}$

${ }^{1}$ University of Kansas, Lawrence, KS, ${ }^{2}$ Harvard Medical School, Boston, MA, ${ }^{3}$, Kansas City, KS

Background/Introduction: Distraction growing rods for early onset scoliosis (EOS) mechanically stabilize the thorax and improve lung function by applying distraction forces at the rib and/or spine. Over-stabilizing can lead to adjacent level complications necessitating subsequent revisions. We investigated how a unilateral growing rod construct implanted in a simulated rib-to-lumbar attachment affects thoracic intradiscal pressure.

Materials/Methods: Five cadaveric thoracic (T1-T12) spine specimens were prepared (three male; mean $68 \pm 3.6$ years (range 65-73)). Standard Needle Tip Pressure Transducers were inserted into T4/T5 and T8/T9 discs. LabVIEW recorded the pressure transducer implementation. Using the FS20 Biomechanical Spine
Test System, specimens were tested in lateral bending, flexion/ extension, and axial rotation with a $400 \mathrm{~N}$ follower load in displacement control $(1 \% \mathrm{sec})$ to a load limit of $\pm 5 \mathrm{Nm}$. After testing the intact thoracic spine, a single growing rod was proximally attached to the $T 5 \mathrm{rib}, 2.5 \mathrm{~cm}$ lateral to the costotransverse joint. The distal attachment was secured to the inferior potting, simulating a rigid joint and attachment to the lumbar spine. The same conditions were used to test the cadaver specimen with the unilateral growing rod construct. Intradiscal pressures of the intact and construct cases were compared using two-tailed paired t-tests $(\mathrm{p}=0.05)$.

Results: There was no significant difference between intradiscal pressure pre- and post-implantation. Average maximum intradiscal pressure at T4/T5 superior adjacent level decreased post-implantation $13 \%$ during axial rotation, $13 \%$ during flexion/extension, and $15 \%$ during lateral bending. Average range of intradiscal pressures changed differently at the superior adjacent level post-implantation. The pressure range increased $9 \%$ during axial rotation, decreased $9 \%$ during flexion extension, and decreased $14 \%$ during lateral bending. Between attachment sites (T8/T9), average maximum intradiscal pressure increased $6 \%$ during axial rotation, $10 \%$ during flexion/extension, and $18 \%$ during lateral bending. Similarly, average range of intradiscal pressure between attachment sites decreased $19 \%$ during axial rotation, $10 \%$ during flexion/extension, and $2 \%$ during lateral bending.

Discussion/Conclusion: Less rigid growing rod constructs do not significantly affect the intradiscal pressure in a cadaver model and may be less likely to cause complications. Less rigid constructs (e.g. unilateral) could be employed in EOS correction to provide adequate stability without causing significant pressure changes.

Poster 4. Complications and Mortality Following 1 to 2 Level Lumbar Fusion Surgery in Patients Above 80 Years of Age

Varun Puvanesarajah, $B S^{1}$, Amit Jain, $M D^{2}$, Adam Shimer, $M D^{l}$, Xudong Li, $M D P h D^{3}$, Anuj Singla, $M D^{3}$, Francis Shen, $M D^{3}$, Hamid Hassanzadeh, $M D^{l}$

', Charlottesville, VA, 2, Baltimore, MD, ${ }^{3}$, Charlottesville, Virginia

Background/Introduction: Patients over eighty years of age represent a substantial proportion of the United States population, with over 11 million such individuals in 2010. Few studies have comprehensively assessed the morbidity associated with spinal fusion surgery in patients older than 80 years. The objective of this study was 1) to determine the 90-day complication rate and 90-day and 1-year mortality in patients 80 years of age and older who were treated with posterolateral lumbar spinal fusion surgery and 2) to compare these rates against those of elderly patients ages 65 to 79 .

Materials/Methods: The PearlDiver database (2005-2012) was utilized to determine morbidity and mortality rates following posterolateral lumbar or lumbosacral spinal fusion surgery of 2-3 vertebrae. Patients $65-79(72,547)$ and $\geq 80$ years old $(12,187)$ were selected. Charlson comorbidity index scores were analyzed and compared, as were various comorbid conditions 90-day complication rates and mortality at 90-days and 1 year were compared between cohorts.

Results: The $\geq 80$ year cohort had a higher average $\mathrm{CCI}$ score than the $65-79$ year cohort (7.99 vs $6.54, \mathrm{p}<0.0001)$. The proportion of patients experiencing at least one major complication was relatively increased by $45.6 \%$ in patients $\geq 80$ year $(13.87$ vs $9.52 \%$; OR $1.5395 \%$ CI $1.44-1.62 \mathrm{p}<0.0001$ ) (Table 1 ). Ninety-day (0.30 vs $0.09 \%$; OR: $3.50,95 \%$ confidence interval: $2.33-5.26, \mathrm{p}<0.0001)$ and one-year $(0.48$ vs $0.18 \%$; OR: 2.58 , $95 \%$ confidence interval: $1.90-3.52, \mathrm{p}<0.0001)$ mortality were 
significantly higher in the $\geq 80$ year cohort compared to the $65-79$ year old control group.

Discussion/Conclusion: Patients 80 years of age or older have significantly greater rates of major medical complications and mortality following 1-2 level lumbar spinal posterolateral fusion surgery compared to patients 65-79 years of age. Patients in this age group should be appropriately counseled and selected to prevent unfavorable outcomes.

Poster 5. Preoperative Anemia as an Independent Risk Factor for Complications Following Elective Adult Spinal Deformity Surgery.

Sulaiman Somani, $B S^{1}$, John Di Capua, MHS, BS ${ }^{1}$, Joung Heon Kim, BS $S^{2}$, Nathan Lee, BS $S^{l}$, Parth Kothari, BS , Samuel Cho, $M D^{3}$

${ }^{1}$ Icahn School of Medicine at Mount Sinai, New York, New York, ${ }^{2}$ Icahn School of Medicine at Mount Sinai, New York, NY, ${ }^{3}, \mathrm{NY}, \mathrm{NY}$

Background/Introduction: Preoperative anemia is of paramount importance in adult spinal deformity surgery (ADS) because it confers an increased risk for high intraoperative blood loss and larger metabolic demands by the spinal cord. Previous literature concurs with this idea, showing anemia to be an independent risk factor for postoperative morbidity and mortality in many surgical procedures. This study examines preoperative anemia as a risk factor in ADS complications.

Materials/Methods: A retrospective cohort analysis was performed on the prospectively collected American College of Surgeons National Surgical Quality Improvement Program (ACS NSQIP) database from 2010 to 2014. Patients ( $\geq 18$ years of age) undergoing ADS were categorized as anemic (males: hematocrit $\leq 39 \%$; females: hematocrit $\leq 36 \%$ ) and non-anemic. Chi-squareand t-tests were used to compare the demographic, surgeryspecific, preoperative comorbidity, and postsurgical complications for both groups. Multivariate logistic regression models were generated to identify independent risk factors.

Results: A total of 5,337 patients met the inclusion criteria, 1,093 $(20.5 \%)$ of which were anemic. Multivariate logistic regressions revealed anemia to be an independent risk factor for the following complications: mortality $(\mathrm{OR}=5.88(2.72,12.60), \mathrm{p}<0.0001)$, length of stay $(\mathrm{OR}=2.00(1.71,2.33), \mathrm{p}<0.0001)$, wound complications $(\mathrm{OR}=2.08(1.43,3.03), \mathrm{p}<0.0001)$, peripheral nerve injury $(\mathrm{OR}=7.69(1.42,42.52), \mathrm{p}=0.0179)$, cardiac complications $(\mathrm{OR}=3.49(1.80,6.79), \mathrm{p}=0.0002)$, postoperative transfusion $(\mathrm{OR}$ $=2.17(1.83,2.55), \mathrm{p}<0.0001)$, sepsis $(\mathrm{OR}=2.08(1.37,3.16), \mathrm{p}$ $=0.0006)$, reoperation $(\mathrm{OR}=1.96(1.44,2.64), \mathrm{p}<0.0001)$, and readmission $(\mathrm{OR}=1.75(1.36,2.26), \mathrm{p}<0.0001)$.

Discussion/Conclusion: Preoperative anemia is an independent risk factor for many postoperative complications, including, but not limited to, wound and cardiac complications, sepsis, length of stay, reoperation, readmission, and mortality.

Poster 6. Age Stratification and Associated Postoperative Complications Following Adult Spinal Deformity Surgery

Sulaiman Somani, $B S^{1}$, Joung Heon Kim, BS ${ }^{2}$, John Di Capua, MHS, BS $S^{l}$, Nathan Lee, BS $S^{1}$, Parth Kothari, BS $S^{1}$, Samuel Cho, $M D^{3}$

${ }^{1}$ Icahn School of Medicine at Mount Sinai, New York, New York, ${ }^{2}$ Icahn School of Medicine at Mount Sinai, New York, NY, ${ }^{3}, \mathrm{NY}, \mathrm{NY}$

Background/Introduction: A continual rise in both the number of spinal surgeries performed and the proportion of elderly patients undergoing elective adult spinal deformity surgery (ADS) necessitate an understanding of age as a risk factor for ADS postoperative complications, which is undertaken in this study.

Materials/Methods: A retrospective cohort analysis was performed on the prospectively collected American College of Surgeons National Surgical Quality Improvement Program (ACS NSQIP) database from 2010 to 2014. Patients ( $\geq 18$ years of age) from the NSQIP database undergoing ADS were separated into age-based cohorts ( $\leq 52,53-61,62-69$, and $\geq 70$ years of age). Age groups were determined by interquartile analysis. Chi-square- and t-tests were used to compare the demographic, surgery-specific, preoperative comorbidity, and postsurgical complications for all four cohorts. Multivariate logistic regression models were generated to identify independent risk factors.

Results: A total of 5,805 patients met the inclusion criteria. Age group $1,2,3$, and 4 contained 1,518 (26.1\%), 1,478 (25.4\%), 1,451 $(25.0 \%)$, and $1,358(23.4 \%)$ patients, respectively. Multivariate logistic regression analysis revealed age, as broken up into the respective cohorts, to be an independent risk factor, for age group 1 relative to age group 4 , in length of stay $(\mathrm{OR}=1.39(1.12,1.69)$, $\mathrm{p}=0.0017)$, all postoperative complications $(\mathrm{OR}=1.64(1.35$, $2.00), \mathrm{p}<0.0001)$, renal complications $(\mathrm{OR}=3.45(1.43,8.33), \mathrm{p}$ $=0.0054)$, UTI $(\mathrm{OR}=2.70(1.49,4.76), \mathrm{p}=0.0009)$, postoperative transfusion $(\mathrm{OR}=1.47(1.20,1.82), \mathrm{p}=0.0002)$, and unplanned readmission $(\mathrm{OR}=1.64(1.18,2.23), \mathrm{p}=0.0040)$, all within 30 days of ADS. Gradations in odds ratios existed between the different cohorts, such that the deleterious effect of age was less pronounced in cohort 3 compared to cohort 4 (relative to cohort 1 ), and even more less so between cohort 2 and cohort 4 (relative to cohort 1).

Discussion/Conclusion: Age has been shown to be an independent risk factor for a variety of postoperative complications, notably in a graded manner such that the greater the age, the greater the risk for the individual of acquiring those postoperative complications.

Poster 7. Perceived Efficacy and Utility of Bioskills Curricula for Resident and Fellow Education

Barrett Boody, M.D. ${ }^{1}$, Brett Rosenthal, MD ${ }^{2}$, Alpesh Patel, MD, FACS ${ }^{2}$, Wellington Hsu, M.D. ${ }^{3}$, Jason Savage, M.D. ${ }^{4}$

${ }^{1}$ Northwestern Memorial Hospital, Chicago, illinois, ${ }^{2}$ Northwestern University, Chicago, IL, ${ }^{3}$ Northwestern Memorial Hospital, Chicago, Illinois, ${ }^{4}$ The Cleveland Clinic, Cleveland, Ohio

Background/Introduction: While bioskills curricula are widely used in orthopaedic and neurosurgical training programs, little is known about the perceived utility and efficacy of these interventions. Bioskills laboratories allow participants to learn and develop complex surgical skills in a controlled environment without the risks of prolonging operation times or potential morbidity to patients. However, how transferable and applicable these bioskill sessions are to the performance of spinal surgery is relatively unknown.

Materials/Methods: An online survey was submitted LSRS members, inviting responses from faculty who educate residents or fellows. Questions were designed to understand residency/ fellow program training experiences as well as the utilization and perceived efficacy of bioskills laboratories.

Results: 36 responses were received, with responding faculty from 25 orthopaedic, 7 neurosurgical, and 4 integrated ortho/ neuro departments. The majority (12/18) of orthopaedic faculty reported residents completing less than 80 spine cases compared to all neurosurgical faculty reporting over 200 spine cases $(n=6)$. Competency for various spine surgical skills trended toward 
earlier level-of-training expectations for neurosurgery compared with orthopaedics. 68\% (17/25) of faculty reported using bioskills for education, using cadavers, sawbones, and software/haptic simulations most frequently $(94 \%, 82 \%, 35 \%$ respectively). Use of bioskills for education was reported by $62.5 \%(10 / 16)$ of orthopaedic and $83.3 \%(5 / 6)$ of neurosurgical faculty $(\mathrm{Z}=0.9328$, $\mathrm{p}=0.3524)$. The majority of respondents $(96 \%, 22 / 23)$ preferred to maintain or increase their program's time spent on bioskills curriculum. When asked if satisfactory completion of bioskills modules would encourage advancing trainees' participation (1-10 rating scale, 10-very greatly increase), overall responses averaged $5.87(\mathrm{n}=23)$. When stratified by department, neurosurgical faculty reported $4.00(n=6)$ versus orthopaedics $(6.43, n=14)(p=0.0139)$. Similarly, orthopaedic faculty were more likely than neurosurgical faculty to attribute resident's/fellow's surgical skills to successful completion of bioskill modules (1-10 rating scale, 6.09 versus 4.00 , respectively, $\mathrm{p}=0.0057$ ).

Discussion/Conclusion: Bioskills laboratories are a widely utilized resource to educate residents and fellows on surgical skills and techniques. While orthopaedic faculty seem to perceive greater efficacy of bioskills laboratories on developing readily transferable surgical skills than their neurosurgical colleagues, this may be partially influenced by variability in orthopedic versus neurosurgical program training experiences.

Poster 8. Perioperative Complications Associated with Posterolateral Spine Fusions: a Study of Elderly Medicare Beneficiaries

Varun Puvanesarajah, $B S^{1}$, Jourdan Cancienne, $M D^{2}$, Brian Werner, $M D^{2}$, Amit Jain, $M D^{3}$, Anuj Singla, $M D^{2}$, Adam Shimer, $M D^{I}$, Francis Shen, $M^{2}$, Hamid Hassanzadeh, $M D^{I}$ MD

, Charlottesville, VA, ${ }^{2}$, Charlottesville, Virginia, ${ }^{3}$, Baltimore,

Background/Introduction: Elderly patients constitute a rapidly growing demographic with an increasing need for spinal procedures. Complication rates for spinal surgery in elderly patients range from $37 \%$ to $80 \%$ with major complications occurring in $12 \%$ to $21 \%$ of patients. The objective of this study was to compare the occurrence of complications in patients treated with 1-2 level, 3-7 level, and greater than 8 level fusions.

Materials/Methods: The PearlDiver database (2005-2012) was utilized to compare perioperative complication rates in patients age 65 years and older undergoing posterolateral fusion of 2-3 (n $=90,186) ; 4-8(n=23,427)$, and 9 or more thoracolumbar vertebral bodies $(n=2,687)$. Cohorts were matched by demographics and comorbidities. Ninety-day medical and surgical complication and mortality rates were determined.

Results: In the full, unmatched cohort, the major complication rate was $15.9 \%$, with matched cohorts of $2-3,4-8$, and $\geq 9$ fused vertebrae associated with major complication rates of $12.3 \%$, $20.9 \%$, and $35.6 \%$, respectively. Patients treated with fusion of $\geq 9$ vertebrae had 3.9 and 2.1 times greater odds of developing a major complication than patients treated with fusion of 2-3 and 4-8 vertebrae, respectively $(\mathrm{p}<0.0001)$. Patients treated with fusion of nine or greater vertebral bodies had 3.6 and 11.5 times increased odds of experiencing mortality than fusions of 4-8 and 2-3 vertebral bodies, respectively (Table 1).

Discussion/Conclusion: Elderly patients treated with spine fusions spanning nine or more vertebrae experience significantly increased complication rates when compared to patients treated with fusions of shorter length.
Poster 9. Anatomical Approach to Determining Surgical Procedure Based on Lumbar Facet Pathomorphology

\section{Mick Perez-Cruet, $M D$}

\section{, Southfield , Michigan}

Background/Introduction: Despite the anatomic understanding of the function of the facet joint (which provides crucial stability to the spine) and its pathomorphology, few studies have illustrated how morphometric analysis of the facet complex preoperatively can help determine favorable surgical approach. Elongation of the facet complex can imply relative instability that is not detected on routine flexion extension dynamic plain X-ray films. Thus preoperative facet morphology analysis can help to determine whether decompression alone versus more extensive decompression, fusion, and instrumentation is needed to treat spinal pathology in the lumbar spine.

Materials/Methods: Morphometric data was gathered from MRI and CT of the lumbosacral spine prior to surgery and analyzed in terms of facet morphology, disease presentation, and severity of progression. Bilateral superior and inferior facets were measured for length and area; total facet joint area was also measured. Operative as well as non-operative levels were measured spanning from L3 to S1. The Student's T-Test was used to evaluate whether or not a significant difference existing between patients who were unaffected at the level, received decompression, or received decompression with fusion and instrumentation. Finally, outcome data was evaluated to determine if these surgical practices had been successful for the individual patients and as a group.

Results: Facet length and area was found to be significantly greater for those patients who required decompression, fusion and instrumentation, compared to those who required decompression alone and normal non-operated levels. Decompression alone showed facet sizes that were markedly similar to normal levels, showing very little hypertrophy. Favorable patient outcomes and a relative lack for redo operations showed this to be a favorable surgical approach strategy.

Discussion/Conclusion: The current study shows how individual facet anatomy can be a strong indicator as to which surgery is most suited for a particular patient. A prospective study should be implemented to better confirm these findings, in which the present methodology would be used. An algorithm was created for deciding surgical intervention by providing a threshold model for facet size, in which significantly elongated facets were a strong indicator for decompression, fusion, and instrumentation.

Poster 10. Proximal Junctional Failure (PJF) Severity Scale Inter- and Intra-observer Reliability

Farbod Rastegar, $M D^{1}$, Alec Contag, $B S^{1}$, Alan Daniels, $M D^{2}$, Eric Klineberg, $M D^{3}$, Justin Smith, $M D^{4}$, Richard Hostin, $M D^{5}, D$. Hamilton, $M D^{6}$, Douglas Burton, $M D^{7}$, Jeffrey Gum, $M D^{8}$, Robert Hart, M.D. ${ }^{9}$

${ }^{1}$ Oregon Health \& Science University, Portland, Oregon, ${ }^{2}$ Department of Orthopaedics, Division of Spine Surgery- Adult Spinal Deformity Service, Warren Alpert Medical School of Brown University, Providence, Rhode Island, ${ }^{3}$ University of California Davis, Sacramento, California, ${ }^{4}$ University of Virginia, Charlottesville, VA, ${ }^{5}$ Baylor Scoliosis Center, Plano, TX, ${ }^{6}$ University of Pittsburgh Medical Center, Pittsburgh, PA, ${ }^{7}$ University of Kansas Medical Center, Kansas City, KS, ${ }^{8}$ Norton Leatherman Spine Center, Louisville, KY, ${ }^{9}$ Oregon Health and Science University, Portland, OR

Background/Introduction: Proximal junctional failure (PJF) is a potentially catastrophic complication following adult spinal 
deformity surgery. No validated classification system exists to help guide management of patients with PJF. PJF severity scale (PJFSS) is a proposed clinical tool to help guide management of patients with PJF. In this study we investigate the repeatability of the PJFSS and its utility in predicting revision surgery for patients experiencing PJF.

Materials/Methods: Sixteen cases selected from the adult spinal deformity database from a single institution were used for scoring based on the PJFSS classification. Six categories were assigned points: neurologic deficit, focal pain, instrumentation problem, change in kyphosis, level of upper instrumented vertebrae (UIV), and UIV/UIV+1 fracture. Each case included the patient's clinical history, preoperative X-ray, details of surgical procedure, postoperative X-rays, post-operative CT, PJK measurements, VAS score and neurologic symptoms. Eleven independent spine surgeons reviewed each category and graded each case based on the PJFSS classification. The scoring process was repeated at two different time intervals at least four weeks apart. Intra-class correlation coefficients were calculated to determine inter- and intra-observer reliability. At the end of each case, participants provided recommendations regarding revision surgery.

Results: Excellent inter-observer reliability was seen in all categories ranging from 0.817 to 0.988 , with the exception of diagnosing fracture at the proximal junction which had only a good inter-observer reliability of 0.692 for the second measurement. There was an excellent average inter-observer reliability of 0.917 regarding recommendation for surgical intervention (Table 1). There was a significantly higher mean PJFSS score for cases where revision surgery was recommended (8.05 vs 5.04 and 8.32 vs 5.27 for measurements 1 and 2 , respectively; p<0.0001).

Discussion/Conclusion: PJFSS is a reliable classification system in guiding management of patient with proximal junctional failure. The PJFSS is also validated as a predictor of recommendation to perform revision surgery for patients experiencing PJF.

Poster 11. Proximal Junctional Kyphosis: Inter- and Intraobserver Reliability in Adult Spinal Deformity

Farbod Rastegar, $M D^{l}$, Alec Contag, $B S^{l}$, Alan Daniels, $M D^{2}$, Jayme Hiratzka, $M D^{I}$, Clifford Lin, $M D^{I}$, Khoi Than, $M D^{I}$, Ahmed Raslan, $M D^{l}$, Christopher Kong, $M D^{l}$, Ngoc-Lam Nguyen, $M D^{l}$, Robert Hart, M.D. ${ }^{3}$

${ }^{1}$ Oregon Health \& Science University, Portland, Oregon, ${ }^{2}$ Department of Orthopaedics, Division of Spine Surgery- Adult Spinal Deformity Service, Warren Alpert Medical School of Brown University, Providence, Rhode Island, ${ }^{3}$ Oregon Health and Science University, Portland, OR

Background/Introduction: Proximal junctional kyphosis (PJK) is commonly seen after adult spinal deformity (ASD) surgery. In this study we evaluated the inter- and intra-observer reliability of PJK measurement in patients who have undergone adult spinal deformity surgery.

Materials/Methods: Fifty-two 36" lateral radiographs were selected of patients following fusion for ASD. The radiographs were divided into four different categories based on the level of upper instrumented vertebra (UIV) and the presence of proximal junctional failure. Seven spine surgeons digitally measured PJK from the upper instrumented vertebra (UIV) to the UIV+2 on two separate sessions at least 6-weeks apart. Intra- and Inter-observer reliability was calculated for each of the four groups.

Results: Combined inter-observer reliability for Round 1 and 2 were 0.955 and 0.983 , respectively. The thoracolumbar without failure group (TL) had inter-observer reliability of 0.965 and 0.975 for round $1 \& 2$, respectively. TL with failure (TLF) group had 0.882 and 0.989 inter-observer reliability for round $1 \& 2$, respectively. The upper thoracic (UT) group had reliabilities of 0.917 and 0.932 , respectively. The UT group with failure (UTF) had inter-observer reliability of 0.956 and 0.958 , respectively. The average intra-observer reliability for TL, TLF, UT and UTF were $0.898,0.975,0.801$ and 0.879 , respectively (Table 1 ).

Discussion/Conclusion: This study demonstrates a high level of inter \& intra-observer reliability in radiographic measurement of proximal junctional kyphosis in patients who have undergone reconstructive surgery for adult spinal deformity at both upper thoracic and thoraco-lumbar end points.

Poster 12. Facial Contamination During Spine Surgery: The Case for Sterile Protection

Christopher Cook, DO ${ }^{1}$, Kris Radcliff, $M D^{2}$, Barrett Woods, $M D^{3}$, Fabio Orozco, $M D^{4}$, Alvin Ong, MD ${ }^{5}$, Mary Scott, RN, $B S N^{6}$, Gabrielle Caucci, $P A-C^{5}$

${ }^{1}$ Inspira Health Network, Philadelphia, Pennsylvania, ${ }^{2}$ Rothman, Philadelphia, PA, ${ }^{3}$ Rothan Institute, Philadelphia, Pennsylvania, ${ }^{4}$ Rothman Institute, Philadelphia, Pennsyvania, ${ }^{5}$ Rothman Institute, Philadelphia, Pennsylvania, ${ }^{6}$ Rothman Institute / Atlanticare , Philadelphia , Pennsylvania

Background/Introduction: Increasing data suggests that surgical site infection in spine surgery occurs as a result of intraoperative inoculation. Although substantial research has been performed on creating a bactericidal environment with antibiotics, there is a paucity of research on the reasons for inoculation. Unlike other areas of orthopedic surgery with a high infection risk, in spine surgery the surgical team typically uses a face mask instead of a space-suit surgical shield. The hypothesis of this study was that debris from the spine surgical field frequently rises to the level of the surgeon's face.

Materials/Methods: Forty-six consecutive elective spine surgeries between May 2015 and August 2015 were included from a single surgeon practice. For each surgery, each member of the surgical team wore a surgical hood with sterile face shield. At the conclusion of each procedure, the face shield would carefully be inspected by two members of the surgical team to identify the presence of any blood, tissue, or burr dust.

Results: The rate of surgeon face shield contamination was 38/46 $(83 \%)$. The rate of first assistant face shield contamination was $(35 \%)$. The scrub tech had a $0 \%$ rate of face shield contamination. The highest contamination rates occurred with TLIF (100\%), open laminectomy and fusion (100\%), and ACDF (93\%).

Discussion/Conclusion: There is a high rate of face shield contamination that occurs during spine surgery. It is possible that blood or burr particles striking the surgeon's face shield represent a potential source of contamination if they ricochet back into the operative field. Spine surgeons may consider using these shields, particularly in high risk cases.

Poster 13. Imaging Measurements For Congenital Lumbar Spinal Stenosis

Chadi Tannoury, $M D^{l}$, Nishant Dwivedi, $B S^{2}$, Falgun Chokshi, $M D^{3}$, Ahmed Moussa, $M D^{4}$, Tony Tannoury, $M D^{5}$, Nadja kadom, $M D^{3}$

${ }^{1}$ Boston University Medical Center, Boston, MA, ${ }^{2}$ boston university, Boston, MA, ${ }^{3}$ Emory, atlanta, georgia, ${ }^{4}$ Ain Shams university (Cairo) \& Boston University, Boston, MA, ${ }^{5}$ Boston University Medical Center, Boston, Massachusetts

Background/Introduction: Congenital lumbar spinal stenosis 
(CLSS) is a developmental narrowing of the lumbar spinal canal causing chronic back pain, radiculopathy, and neurogenic claudication. It presents with neurogenic claudication at an earlier age than patients without CLSS. We assessed 3 measurement methods on radiographs and cross-sectional imaging for inter-rater reproducibility and for correlations with a clinical diagnosis of CLSS versus degenerative disease or lumbar pain in the absence of radiographic abnormality. The goal of this study was to determine the best measurement technique for a diagnosis of CLSS.

Materials/Methods: Three measurement methods of CLSS were evaluated in 1) 30 patients with symptomatic CLSS, 2) 30 patients without CLSS and with degenerative lumbar disease, and 3) 30 patients presenting with back pain in the absence of imaging pathology. Method 1: Ratio of anterior-posterior (AP) vertebral body (VB) diameter over spinal canal AP diameter at L3 on radiographs and MRI; Method 2: Ratio of VB and spinal canal cross-sectional area at L3 on MRI; Method 3: Measurement of the L2-S1 lumbosacral angle on radiographs. Statistics: Inter-rater reliability and regression (Stata 13.1, Texas, USA).

Results: All measurement methods showed almost perfect reproducibility (intra-class $r>0.81$ ). CLSS had expected greater association with male gender $(\mathrm{p}=0.023)$ and younger age $(\mathrm{p}=0.007)$. BMI was highest in patients with CLSS and showed a trend $(\mathrm{p}=0.057)$. The measurement methods that best correlated with a diagnosis were method $2(\mathrm{p}=0.012)$ and method $3(\mathrm{p}=0.009)$, showing that patients with a diagnosis of CLSS had the smallest cross-sectional imaging ratios and the smallest Cobb angles on radiographs.

Discussion/Conclusion: 1. The best imaging measurement methods for a diagnosis of CLSS are the ratio of vertebral body area over spinal canal area on MRI (method 2) and the lumbosacral angle between L2 and S1 on radiographs (method 3). 2. As expected, CLSS was associated with male gender and younger age. BMI may play a role in CLSS, but did not reach significance in our study.

Poster 14. Complications and Mortality after Lumbar Spinal Fusion in Elderly Patients with Late Stage Renal Disease

Varun Puvanesarajah, $B S^{1}$, Amit Jain, $M D^{2}$, Daniel Hess, $M D^{3}$, Adam Shimer, $M D^{l}$, Francis Shen, $M D^{3}$, Hamid Hassanzadeh, $M D^{l}$

${ }^{1}$, Charlottesville, VA, ${ }^{2}$, Baltimore, MD, ${ }^{3}$, Charlottesville, Virginia

Background/Introduction: Lumbar spinal fusion surgeries are common in elderly patients, who are well-known to have increased comorbidity burden. Elderly patients with severe chronic kidney disease (CKD) represent a population with poorly understood mortality and complication rates following spine surgery. It is important to study these undesired sequelae to improve patient education and risk stratification. The objective of this study was to assess complication and mortality rates following lumbar spinal fusion surgery in patients with late stage renal disease.

Materials/Methods: Medicare data from the PearlDiver Database (2005-2012) was queried for patients who underwent primary 1-2 level posterolateral lumbar spine fusion surgeries. This cohort was divided into two study groups: a cohort with a pre-existing diagnosis of late stage renal disease $(\mathrm{N}=1,654)$ and a control cohort of all other patients $(\mathrm{N}=242,085)$. The control group was matched to the renal disease cohort by age, gender, and comorbidities. 90-day complication rates and 90-day and 1-year mortality were assessed.

Results: The renal disease cohort had increased rates of all medical complications (21.3 vs $14.2 \%$; OR $1.6495 \%$ CI $1.44-$ $1.85 \mathrm{p}<0.0001)$. Increased rates of infection (4.4 vs $1.8 \%$; OR
$2.4395 \%$ CI $1.87-3.16 \mathrm{p}<0.0001)$ and procedure-day blood transfusions (20.7 vs 14.7\%; OR $1.5195 \%$ CI $1.33-1.72 \mathrm{p}<$ 0.0001 ) were also observed in the renal disease cohort (Table 1). Both ninety-day (1.1 vs $0.2 \%$; OR: $5.05,95 \%$ confidence interval: $2.90-8.77, \mathrm{p}<0.0001)$ and one-year mortality (1.9 vs $0.7 \%$; OR: $2.77,95 \%$ confidence interval: $1.87-4.11, \mathrm{p}<0.0001)$ were significantly higher in the renal disease group compared to the control group (Table 1).

Discussion/Conclusion: Elderly patients with late-stage renal disease treated with 1-2 level posterolateral lumbar fusion have 1.6 times increased odds of experiencing a major medical complication within three months of surgery and 2.8 times increased odds of one-year mortality when compared to matched controls. Elderly patients with late-stage renal disease should be appropriately counseled of these risks prior to surgery

Poster 15. Association of Low Perioperative Prealbumin Level and Surgical Complications in Long Segment Spinal Fusion Patients

Jian Guan, MD, Christopher Holland, MD, PhD, Meic Schmidt, MD, MBA; Andrew Dailey, MD, Mark Mahan, MD, Erica Bisson, $M D, M P H$

\section{University of Utah, Salt Lake City, UT}

Background/Introduction: The significant impact of malnutrition on surgical outcomes has been well established in many surgical specialties. The objective of this study is to evaluate the perioperative nutritional status in patients undergoing long segment spinal fusion operations and its relationship to surgical outcomes and complication rates.

Materials/Methods: A retrospective review of a comprehensive clinical database was utilized to identify patients who underwent long segment $(\geq 7)$ spinal fusions in whom a serum prealbumin level was obtained within 10 days of the surgical date. All demographic, clinical, operative, and postoperative parameters were collected and examined. Prealbumin levels were dichotomized based on a threshold value of $11 \mathrm{mg} / \mathrm{dL}$ into low and high groups.

Results: A total of 47 patients were identified with a mean prealbumin level of $12.2 \mathrm{mg} / \mathrm{dL}$, with 20 patients $(43 \%)$ in the low group and 27 patients $(57 \%)$ in the high group. The primary diagnosis was scoliosis and spinal deformity. Patients with low prealbumin were more likely to undergo a longer fusion (11.6 versus 9.1 levels, $\mathrm{p}=0.003$ ) and to undergo a two-staged procedure $(35 \%$ vs. $11 \%, p=0.048)$. Patients in the low prealbumin group were significantly more likely to develop an infectious complication than those in the higher prealbumin group (50\% versus $15 \%$, $\mathrm{p}=0.009)$, most commonly urinary tract infection $(10 / 14,71 \%)$. There was no significant difference in rates of non-infectious complications between the two groups. Multivariate analysis revealed only low prealbumin to be significantly associated with postoperative infection (OR 6.673, 95\% CI 1.236-36.024).

Discussion/Conclusion: Low prealbumin levels are associated with higher rates of non-wound related infections in patients undergoing long-segment posterior spinal fusion procedures. Further investigations are needed to better define this association and to determine whether effective interventions may be implemented among this subset of patients to attenuate this relationship. 
Poster 16. Correlations Between Quantitative T2 and T1@ MRI Parameters and Mechanics and Biochemical Content in a Rabbit Intervertebral Disc Degeneration Model

Sarah Gullbrand, $P h D^{l}$, Beth Ashinsky, BS ${ }^{2}$, John Martin, PhD ${ }^{2}$, Stephen Pickup, $P h D^{2}$, Lachlan Smith, PhD ${ }^{2}$, Robert Mauck, $P h D^{2}$, Harvey Smith, $M D^{2}$

\section{${ }^{1}$, Philadelphia, PA, ${ }^{2}$, Philadelphia, Pennsylvania}

Background/Introduction: Improved diagnostic measures for intervertebral disc degeneration are necessary to enable early detection and treatment. Quantitative MRI, such as T2 and T1@ mapping, may serve as powerful non-invasive diagnostic techniques to evaluate compositional and functional changes to the intervertebral disc (IVD). The aim of this study was to correlate changes in mechanical and biochemical properties with MRI parameters in rabbit IVDs using an in vitro chymopapain digestion model.

Materials/Methods: Rabbit lumbar spine motion segments ( $\mathrm{n}=5$ per group) were injected with $100 \mu \mathrm{L}$ of saline (control) or chymopapain at $3 \mathrm{U} / \mathrm{mL}, 15 \mathrm{U} / \mathrm{mL}$ or $100 \mathrm{U} / \mathrm{mL}$. Following overnight digestion, T2 and T10 MRI series were obtained at 4.7T. Specimens were mechanically tested in tension-compression (20 cycles, $+21 \mathrm{~N}$ to $-42 \mathrm{~N})$ and creep $(10$ minutes, $-42 \mathrm{~N})$, to obtain the neutral zone (NZ) modulus, NZ range of motion (ROM), compressive modulus, compressive ROM, total ROM, creep moduli $(\mathrm{S} 1, \mathrm{~S} 2)$ and creep time constants $(\tau 1, \tau 2)$. NP water and GAG contents were quantified and normalized to total NP volume. A stepwise multiple linear regression was performed to determine which parameters contributed most to changes in NP T2 and T1@ values.

Results: Chymopapain digest reduced NP T2, T1@, GAG and water content in a dose-dependent manner (Fig 1). Changes in disc mechanical properties were also directly affected by increasing chymopapain dosage, with the exception of the $100 \mathrm{U} / \mathrm{mL}$ group. When data from all groups were included, stepwise multiple regression yielded a model with GAG, compressive modulus, $\tau 1$ and $\tau 2$ as variables significantly $(\mathrm{p}=0.006)$ affecting $\mathrm{T} 2(\mathrm{r} 2$ $=0.64$ ). Stepwise regression identified GAG and NZ modulus as variables contributing to $\mathrm{T} 1 \mathrm{Q}(\mathrm{p}=0.08, \mathrm{r} 2=0.64)$. When the $100 \mathrm{U} / \mathrm{mL}$ group (advanced degeneration), was excluded from the multiple regression analysis, T2 was found to be significantly impacted by compressive modulus, $\tau 1$ and water content $(p=0.009$, $\mathrm{r} 2=0.71$, while no variables correlated significantly in the model for $\mathrm{T} 1 \mathrm{\varrho}$.

Discussion/Conclusion: These results indicate that quantitative MRI can detect changes in the mechanical and biochemical properties of the IVD. T2 may be more sensitive to early stage degenerative changes than T1@, while both quantitative MRI parameters are sensitive to advanced degeneration.

Poster 17. A Novel Method Using Surgimap Software to Predict Sagittal Alignment Following Pedicle Subtraction Osteotomy

Robert Merrill, $B S^{1}$, Jun Kim, $M D^{2}$, Joshua Meaike, $B S^{2}$, Dante Leven, DO ${ }^{3}$, Joung Heon Kim, BS ${ }^{4}$, Samuel Cho, $M D^{3}$

${ }^{1}$ Mount Sinai Medical Center, New York, NY, ${ }^{2}$, New York, NY, ${ }^{3}$, NY, NY, ${ }^{4}$ Icahn School of Medicine at Mount Sinai, New York, NY

Background/Introduction: Adult spinal deformity is associated with sagittal misalignment and negative health related quality of life. When conservative management of adult spinal deformity fails, surgical intervention is often the remaining option. Pedicle subtraction osteotomy (PSO) is a technique that attempts to restore sagittal alignment by creating lordosis in the spine. Few validated methods exist for visually planning and predicting the sagittal outcome following PSOs. The computer software Surgimap may be a reliable tool for predicting such an outcome. Our objective is to evaluate the predictive capability of Surgimap and present a novel method for predicting sagittal alignment following PSO.

Materials/Methods: This is a retrospective review of 13 patients who underwent a single level lumbar PSO at Mount Sinai Hospital between 2011-2015. Sagittal alignment measurements listed in table 1 were performed using Surgimap on pre-operative and post-operative standing radiographs. An osteotomy at the operative level was simulated and the films rotated to match the simulated sagittal vertical axis (SVA) to the post-operative SVA. The simulated films were compared to the post-op films for accuracy, and a linear regression was performed for the relationship of pre-operative T1 spino-pelvic inclination (T1 SPI) and SVA to the angular rotation needed to match simulated to actual SVA.

Results: Our 13 patients included 5 men and 8 women with a mean age of 63years \pm 11.35 . The average pre-op T1 SPi, T1 Pelvic Angle (TPA), and SVA were $5.31^{\circ} \pm 9.19,30.54^{\circ} \pm 9.74$, $130.80 \mathrm{~mm} \pm 84.22$, respectively. Average actual outcomes for T1 SPI $\left(-3.23^{\circ} \pm 4.32\right)$, TPA $\left(14.54^{\circ} \pm 8.24\right)$, and SVA $(26.83 \mathrm{~mm}$ \pm 45.67) were not statistically different from predicted $\mathrm{T} 1 \mathrm{SPi}$ $\left(-2.00^{\circ} \pm 4.78\right)$, TPA $\left(12.38^{\circ} \pm 9.84\right)$, and SVA $(26.81 \mathrm{~mm} \pm 45.56)$ Significant differences between actual and predicted outcome were seen only with thoraco-lumbar alignment $(\mathrm{p}=0.0317)$, thoracic kyphosis (0.0211), and T9 SPi (0.0410). There was a significant correlation between angular rotation of the film and pre-op SVA (R2=0.715, $\mathrm{P}=0.0003)$ and T1 SPi $(\mathrm{R} 2=0.729, \mathrm{P}=0.0002)$.

Discussion/Conclusion: Our surgimap algorithm for PSO prediction provides a visual depiction of sagittal correction for T1 SPi, TPA, SVA, and spinopelvic parameters, but may not accurately portray thoracic alignment following PSO.

Poster 18. Comparison of Perioperative Adverse Event Rates after Single-level Transforaminal Lumbar Interbody Fusion versus Anterior/Posterior Fusion for Spondylolytic Spondylolisthesis: 30 Day Outcomes of 635 cases

Matthew Webb, $A B^{1}$, Adam Lukasiewicz, MScl, Andre Samuel, $B B A^{l}$, Bryce Basques, $M D^{2}$, Daniel Bohl, MD, MPH ${ }^{3}$, Michael Daubs, $M D^{4}$, Jonathan Grauer, $M D^{5}$

${ }^{1}$ Yale School of Medicine, New Haven, CT, ${ }^{2}$ Rush University Medical Center, Chicago, IL, ${ }^{3}$ Rush University Medical Center, New Haven, CT, ${ }^{4}$, Las Vegas, Nevada, ${ }^{5}$, New Haven, CT

Background/Introduction: There is no clear consensus whether transforaminal lumbar interbody fusion (TLIF) or anterior/posterior $(\mathrm{A} / \mathrm{P})$ fusion is preferable for surgical treatment of spondylolytic spondylolisthesis. In order to investigate this, we used a large national database to compare perioperative adverse events.

Materials/Methods: The American College of Surgeons National Surgical Quality Improvement Program database (ACSNSQIP) records perioperative adverse events for 30 days after surgery regardless of discharge status. Cases of spondylolytic spondylolisthesis were identified using International Classification of Disease, Ninth Edition code 756.12. Cases treated with TLIF and A/P fusion were then identified using CPT codes. Single-level cases were isolated by excluding cases that included CPT codes for additional levels. Fisher's exact and Perason's chi-squared tests were used to compare perioperative adverse event rates where appropriate. Data management and analyses were performed using Stata/IC 13.0 (StataCorp, College Station, TX). 
Results: In total, 1274 patients were identified: 899 underwent single-level TLIF, 150 underwent single-level A/P fusion, and 225 underwent anterior fusion alone. Of the adverse events recorded in ACS-NSQIP, 19 occurred at least once in one of the groups. Therefore, the level of significance was adjusted to $\mathrm{p}=0.003$ according to Bonferroni's correction. There were 81 adverse events among patients who underwent TLIF (9.0\%), 20 among those who underwent A/P fusion (13.3\%), and 24 among those who underwent anterior fusion alone. This difference was not significant $(\mathrm{p}=0.230)$. The rates of serious adverse events were similar between groups $(4.8 \%, 8.0 \%, 5.3 \%$, respectively, $\mathrm{p}=0.263)$ as were the rates of minor adverse events $(4.2 \%, 5.3 \%$, $5.3 \%$, respectively, $\mathrm{p}=0.687$ ). The rates of postoperative blood transfusions were greater in the $\mathrm{A} / \mathrm{P}$ fusion group $(13.7 \%, 31.3 \%$, and $10.9 \%, \mathrm{p}<0.001)$. The rates of individual adverse events are reported and compared in Table 1.

Discussion/Conclusion: TLIF or A/P fusion for spondylolytic spondylolisthesis were found to have similar adverse event rates at 30 day follow up based on records of the 635 cases in this national database. Because perioperative adverse event rates were similar, these findings suggest that surgeon preference and longer term patient outcomes should be the driver of the recommendation for one approach or another.

Poster 19. Fracture Gap Size Matters: Comminuted Sacral Zone II Fractures with Anterior Instability - Role of Lumbopelvic Fixation in Biomechanical Stabilization?

Ehsan Jazini, $M D^{l}$, Noelle Klocke, $M S^{2}$, Oliver Tannous, $M D^{l}$, Tristan Weir, $B S^{l}$, Herman Johal, $M D, P h D^{l}$, Daniel Gelb, $M D^{l}$, Jascone Nascone, $\mathrm{MD}^{l}$, Robert $\mathrm{O}^{\prime}$ Toole, $\mathrm{Md}^{l}$, Brandon Bucklen, $P h D^{2}$, Steven Ludwig, $M D^{l}$

\section{${ }^{1}$, Baltimore, $\mathrm{MD},{ }^{2}$, Audubon, PA}

Background/Introduction: To determine whether extending lumbopelvic fixation (LPF) to L4 provides stabilization equivalent to that attained by traditional fracture stabilization techniques in a biomechanical model for the treatment of comminuted zone II sacral fractures with anterior instability.

Materials/Methods: Fresh-frozen, cadaveric lumbopelvic specimens $(\mathrm{n}=7)$ were carefully cleaned and potted at L4 and at the left ischium. Intact flexion-extension (FE) motion $(\leq 8 \mathrm{Nm})$ was captured in a bilateral stance using a "floating hip model." Assessment of relative Eulerian motion between iliac crests for the pelvic ring and across the intended sacral fracture zone. Sacral fracture zone motion (SFM) and pelvic ring motion (PRM) reported as an average of intact motion. Pubis symphysis was transected and created a $1 \mathrm{~cm}$ gap through the right sacral foramen. The traditional construct $(T: n=6)$ consisted of an anterior pubis symphysis plate and two trans-sacral screws. LPF was performed by using bilateral cannulated iliac screws and rods (with cross-connectors) to L5 (L5-P: $n=5$ ) or L4 (L4-P: $n=5$ ) pedicle screws. Traditional and LPF techniques were combined into constructs (L5-P+T: $n=7, L 4-P+T: n=6)$. One-way ANOVA revealed significant differences $(p<0.05)$ between normalized FE motion profiles of different constructs.

Results: Both lumbopelvic constructs improved SFM significantly ( $\mathrm{L} 4-\mathrm{P}=71 \%$ and $\mathrm{L} 5-\mathrm{P}=74 \%, \mathrm{p}<0.05)$ compared to traditional fixation alone ( $\mathrm{T}=128 \%$, Figure 1$)$. The combination of traditional and L4-P fixation ( $\mathrm{L} 4-\mathrm{P}+\mathrm{T}=34 \%$ ) significantly improved stability across the sacral fracture compared to individually. With respect to PRM, extension to L4 was required for LPF to have similar stabilization as traditional means, though this was not statistically significant. The combination of lumbopelvic with traditional fixation significantly improved PRM (L4-P+T $=47.9 \%$, $\mathrm{L} 5-\mathrm{P}+\mathrm{T}=54.4 \%$ ) compared to L5-P fixation alone.
Discussion/Conclusion: This study presents strong evidence that comminuted complex sacral fractures with anterior instability LPF can provide significant stability across the sacral fracture zone and may be critical. Extension of the LPF to L4 in a hybrid approach may be needed in highly comminuted cases where maximum biomechanical support is needed across the fracture zone. Extension of LPF to L4 also appears to play an important role in providing pelvic ring stability although no significant added benefit was found when combined with traditional fixation approach.

Poster 20. Early Subspecialization in Orthopedic Spine Surgery Training

J. Mason DePasse, $M D^{l}$, Mark Palumbo, $M D^{2}$, Robert Hart, M.D. ${ }^{3}$, Alan Daniels, $M D^{4}$

${ }^{1}$ Department of Orthopaedics, Warren Alpert Medical School of Brown University, Providence, Rhode Island, ${ }^{2}$ Department of Orthopaedics, Division of Spine Surgery, Warren Alpert Medical School of Brown University , Providence, RI, ${ }^{3}$ Oregon Health and Science University, Portland, OR, ${ }^{4}$ Department of Orthopaedics, Division of Spine Surgery- Adult Spinal Deformity Service, Warren Alpert Medical School of Brown University, Providence, Rhode Island

Background/Introduction: Current Accreditation Council for Graduate Medical Education (ACGME) guidelines require only 15 spine procedures during orthopaedic surgery residency. Neither the ACGME nor the American Board of Orthopaedic Surgery (ABOS) mandate time on a spine service. A high degree of variability exists in spine training during residency, and exposure to this subspecialty may be inadequate for some trainees. This investigation seeks to determine whether trainees planning a career in spine surgery obtain additional spine operative exposure during residency.

Materials/Methods: The chosen fellowships and case logs of all residents graduating from a single orthopedic residency training program from 2002 to 2017 were reviewed. Operative case numbers for any field chosen by more than five residents (including spine surgery, hand surgery, foot and ankle surgery, and sports medicine) were analyzed. The number of cases logged by residents who chose a specific specialty was then compared to the number of cases of residents who chose other specialties.

Results: The case logs of 84 residents, including 74(88.1\%) men and 10(11.9\%) women, graduating from 2002 to 2017 were reviewed. Residents who chose spine surgery as a career performed significantly more spine cases than those who chose subspecialties other than spine surgery $(76.3 \pm 28.8$ vs. $45.7 \pm 17.6, p=0.003)$. In contrast, for hand surgery $(271.9 \pm 112.6$ vs. $231.2 \pm 95.6, \mathrm{p}=0.18)$, sports medicine $(290.6 \pm 66.4$ vs. $259.9 \pm 75.3, \mathrm{p}=0.13)$ and foot and ankle surgery $(163.4 \pm 46.8$ vs. $164.5 \pm 51.4, \mathrm{p}=0.94)$, there was no significant difference in number of cases between residents who chose the subspecialty and those who did not.

Discussion/Conclusion: Residents interested in spine surgery obtained additional spine operative exposure during residency compared to trainees who choose subspecialties other than spine surgery. The ACGME and ABOS mandate only 36 months of specified orthopaedic training after intern year, leaving 12 months to the discretion of individual programs. For orthopaedic residents interested in pursuing spine surgery, discretionary time dedicated to additional spine rotations during training could enhance their preparation in spine surgery. A similar approach could be developed in other orthopaedic subspecialties. 
Poster 21. Single-Center, Consecutive Series Study of the Use of a novel Beta-tricalcium Phosphate and Platelet-Rich Fibrin Matrix as a Bone Graft Substitute in Posterolateral Lumbar Fusion

Brendon Walker, $B S^{1}$, Craig Steiner, $M D^{2}$, Tucker Callanan, $B S^{2}$, Celeste Abjornson, $P h D^{3}$, William Long, BA ${ }^{4}$, Frank Cammisa, $M D^{3}$

${ }^{1}$ Hospital for Special Surgery, New York, NY, ${ }^{2}$, New York, NY, ${ }^{3}$ Hospital for Special Surgery, New York, New York, ${ }^{4}$ Rush University Medical Center, Chicago, IL

Background/Introduction: The use of bone graft substitutes and extenders as alternatives to autogenous iliac crest bone graft (ICBG) in lumbar spine fusion is increasingly common to avoid complications associated with ICBG harvest. Beta-tricalcium phosphate $(\beta-\mathrm{TCP})$ bone graft substitute is engineered to support bone growth and resorb at a rate consistent with new bone deposition. Growth factors derived from platelets have been shown to stimulate many cell types, including lines active in bone formation. The purpose of this retrospective study is to assess the radiographic fusion rate of an FDA approved $\beta$-TCP bone graft substitute in concert with a concentrated platelet formulation in posterolateral lumbar spine fusion.

Materials/Methods: Forty-five consecutive patients underwent posterolateral lumbar fusion (PLF) at a total of sixty operated levels using $\beta$-TCP (Integra Mozaik ${ }^{\mathrm{TM}}$, Plainsboro, $\mathrm{NJ}$ ) combined with platelet-rich fibrin matrix (PRFM) (Vertical Spine Fibrinet ${ }^{\circledR}$ Vertical Spine, Red Bank, NJ) and local bone were reviewed. These procedures included one-level to three-level fusions, performed by a single surgeon. Each operated level was assessed individually. Radiographic fusion results were assessed using the Lenke scoring system (A-D) for assessment of posterolateral bone formation on one year postoperative radiographs.

Results: A total of forty-five patients underwent PLF using the $\beta$-TCP with PRFM bone graft substitute on 60 operated levels. Radiographic fusion was observed at 52 levels $(86.7 \%)$ Thirtythree levels were graded as A $(55 \%)$, nineteen were graded B $(31.7 \%)$, seven were graded C $(11.7 \%)$, and one was graded D $(1.7 \%)$. None of the patients required a revision surgery at the level where the bone graft material was used.

Discussion/Conclusion: The use of this formulation of $\beta$-TCP and PRFM for PLF results in a rate of fusion similar to those reported using ICBG (range 54-94\%) while eliminating the complications associated with ICBG harvesting.

Poster 22. A Comparison Of Clinical Outcomes To Assess Coflex-FTM As An Alternative To The Traditional Pedicle Screw Construct In Augmenting Lumbar Interbody Fusion

Fred Xavier, $M D / P h D^{l}$, Sam Miller, $B S^{2}$, Brendon Walker, $B S^{l}$, Samuel Grinberg, $B A^{3}$, Tucker Callanan, $\mathrm{BS}^{4}$, Frank Cammisa, $M D^{3}$, Celeste Abjornson, $P h D^{3}$

${ }^{1}$ Hospital for Special Surgery, New York, NY, ${ }^{2}$, Providence, RI, ${ }^{3}$ Hospital for Special Surgery, New York, New York, ${ }^{4}$, New York, NY

Background/Introduction: With an aging population, spinal fusions are being performed in greater frequency to treat the degenerative changes and provide pain relief. Newer minimally invasive approaches such as interlaminar fusion (IF) procedures have gained interest and produced satisfactory results. The purpose of this retrospective study is to confirm the ability of IF to provide similar outcomes to the traditional pedicle screw constructs.

Materials/Methods: A series of 116 patients, treated between
2008 and 2014 at our institution, received a singlelevel extreme lumbar lateral interbody fusion (XLIFTM, Nuvasive, San Diego, CA) with posterior stabilization. Fortyfour underwent interlaminar fusion (CoFlexF, Paradigm Spine, New York, NY) and the other 72 had pedicle screw instrumentation, either unilateral or bilateral. Fusion was radiographically assessed preoperatively and at followup (mean: 9.7 months). The evaluated measures included changes in disc height and foraminal height as observed on standing films, as well as changes in motion at the level of intervention as determined by comparing differences in Cobb angles on flexion/ extension images. EBL, operative time, length of stay, the need for an additional operation, and date of last prescription for narcotics were also analyzed.

Results: Both treatment options increased foraminal height ( $\mathrm{p}$ $<0.01)$ as well as disc height $(\mathrm{p}<0.01)$ while yielding decreased motion at the instrumented level $(\mathrm{p}<0.01)$ compared to preoperative values. There were no differences in foraminal height and decreases in motion between the two interventions $(p=0.59$ and $\mathrm{p}=0.68$, respectively). Patients with pedicle screws had a significant greater increase in disc height than those with IF ( $p$ $<0.01)$. However, IF produced lower EBL $(\mathrm{p}<0.01)$, LOS $(\mathrm{p}<$ $0.05)$, reoperation rate $(\mathrm{p}<0.05)$, and time on narcotics $(\mathrm{p}<0.01)$ while experiencing similar operative time $(p=0.51)$ compared to pedicle screw instrumentation.

Discussion/Conclusion: These results indicate that IF serves as a successful posterior stabilization device in the augmentation of onelevel lateral lumbar interbody fusion. Fusion quality and rate were confirmed to be similar between patients. However, the lower morbities associated with IF may lead to better recovery and quality of life.

Poster 23. Vertebral Insufficiency Fractures After ShortSegment Lumbrosacral Fusion: Case Series and Systematic Review of Literature

Scott Mitchell, BS ${ }^{1}$, William Robinson, $M D^{2}$, Ahmad Nassr, $M D^{2}$, Bradford Currier, M.D. ${ }^{3}$, Paul Huddleston III, MD ${ }^{2}$, Brett Freedman, M.D. ${ }^{3}$

${ }^{1}$ University of Kansas Medical Center, Westwood, Kansas, ${ }^{2}$ Mayo Clinic, Rochester, MN, ${ }^{3}$, Rochester, MN

Background/Introduction: Vertebral insufficiency fractures are a relatively rare complication following short segment lumbrosacral fusion that is an often overlooked and underdiagnosed source of acute pain in the post-operative period. Sacral and vertebral insufficiency fractures are usually indications of poor bone mineral density and correlate with DEXA-measured osteoporosis in $>95 \%$ of patients. Most insufficiency fractures are atraumatic, not detected by conventional radiographs, and are often undiagnosed for weeks to months.

Materials/Methods: A systematic Medline search was conducted looking for case reports and case series of short segment (L4-S1 and/or L5-S1) lumbrosacral fusions complicated by vertebral insufficiency fractures. A case series of patients with this diagnosis was compiled at our institution as well. Age, gender, segments, surgical fixation, medical history, bone mineral density, time to fracture, and treatment method were recorded.

Results: A literature reviewed showed 11 cases in the literature of insufficiency fracture following short segment lumbrosacral fusion, and five more cases were recently seen at our institution. Most of the cases have a new onset sacral insufficiency fracture in the early post-operative period (range: 1 day-4 months, median: 31 days). The majority of patients requiring further spinal fixation had ALIF without posterior fixation (7/10), whereas all patients successfully treated nonoperatively had posterior fixation $(6 / 6)$. Indications for surgery included new onset neurologic 
deficit, recurrence of original deformity, and intractable pain after exhaustion of nonoperative measures. Successful nonsurgical management included osteoporosis treatment, lumbrosacral orthotics, encouraged ambulation, and pain control.

Discussion/Conclusion: Vertebral insufficiency fracture is a rare complication following short-segment lumbrosacral fusion that is often missed. These types of fractures are more amenable to nonoperative treatment if the preceding surgery had posterior fixation. The majority of these fractures occurred in patients with no previous diagnosis of bone mineral abnormalities, and a high index of suspicion should be reserved for acute pain in the early post-operative period even in young patients without obvious causes of osteoporosis.

Poster 24. Longitudinal Characterization of Vertebral Bony Remodeling Following Puncture of the Annulus Fibrosus as a Model of Intervertebral Disc Degeneration

Tristan Maerz, Ph.D., Michael Newton, B.S., Abigail Davidson, B.S., Michael Planalp, MD, Jeffrey Fischgrund, MD; Kevin Baker, $\underline{P h . D}$

\section{, Royal Oak, Michigan}

Background/Introduction: Degenerative disc disease (DDD) is accompanied by a myriad of changes within the vertebral motion segment. Characterization in preclinical models could provide a better understanding of the progression of these changes. The objective of this study was to characterize longitudinal changes to lumbar spinal motion segments in a rat annular puncture model of IVD degeneration.

Materials/Methods: Under an approved protocol, 8 Lewis rats underwent baseline $\mu \mathrm{CT}$ imaging. In 4 animals, IVD degeneration was induced via annular puncture at L3-L4 and L5-L6. 4 animals underwent sham surgery. Animals underwent live $\mu \mathrm{CT}$ imaging of motion segments every 2 weeks until the 12 week endpoint. Bone volume fraction (BV/TV), BMD, TMD, trabecular thickness (Tb. Th.), trabecular spacing (Tb.Sp), and trabecular number (Tb.N) were analyzed in the cranial vertebral body. The bony endplate (B-EP) was analyzed via BV/TV, TMD, and total volume (TV). Results were normalized to baseline and compared between groups, while excised spines underwent processing for later histologic analyses.

Results: Animals with a disc puncture exhibited reduction in $\mathrm{BV} / \mathrm{TV}$ and TMD between baseline and 2 weeks followed by a steady increase up to 10 weeks, and exhibited a significantly greater change in TMD at 4 and 6 weeks. (Fig 1D, E). There was a significant difference in the change in Tb.N between the groups at 2 weeks, followed by a steady decline. In the B-EP, annular punctured animals exhibited significantly higher change over baseline in BV/TV at 4 and 6 weeks, TMD at 6 weeks, and lower TV at 4 weeks. Time-dependent morphological changes are evident on $\mu \mathrm{CT}$ images (Fig 1A-C), most notably a loss of disc height, anterior osteophyte formation, and trabecular thickening.

Discussion/Conclusion: Injury to the IVD results in a remodeling response within the bony endplate and vertebral trabecular network. A disuse-phenomenon is observed in both groups at 2 weeks due to abdominal surgery, and evidence of chronic morphometric changes observed thereafter. Chronic remodeling affects biomechanical and biochemical properties of vertebral bone, which has been hypothesized as a major contributor to progression of degeneration. Longitudinal characterization of early and chronic bony remodeling can further our understanding of DDD, while identifying therapeutic targets.
Poster 25. The Effects of Conflicts of Interest on Practice Patterns and Complication Rates in Lumbar Spine Surgery

Ralph Cook, $B S^{l}$, Joseph Weiner, $B S^{2}$, Michael Schallmo, $B S^{2}$, Sameer Singh, $B S^{1}$, Kathryn Barth, $B S^{1}$, Wellington Hsu, M.D. ${ }^{3}$

${ }^{1}$ Northwestern University Department of Orthopaedic Surgery, Chicago, IL , ${ }^{2}$ Northwestern University Department of Orthopaedic Surgery, Chicago , IL, ${ }^{3}$ Northwestern Memorial Hospital, Chicago, Illinois

Background/Introduction: Recent demands from Congress and the Institute of Medicine have highlighted the importance of conflict of interest among physicians. Previous studies have identified orthopedic surgeons as receiving among the highest industry payment amounts. However, to date, no study has investigated the association or potential effects of disclosed industry payments with quality of patient care. We sought to determine whether financial relationships with industry had any impact on practice patterns or complication rates of spine surgeons.

Materials/Methods: A comprehensive database of spine surgeons in the United States with compiled data of industry payments, practice patterns, and complication rates was created. Practice pattern data was derived from a publically-available Medicare-based database generated from CPT codes which included the total number of and rate at which each spine surgeon recommends fusion (2011-2012). Complication rate data for each surgeon was extracted from the ProPublica Surgeon Scorecard database (2009-2013) (https://projects.propublica.org/surgeons/), which utilizes in-hospital mortality and readmission within 30 days of discharge as likely complications of surgery. A mixedeffects model adjusting for age and health-status along with hospital and surgeon random-effects was used to risk-adjust each surgeon's raw complication rate. Data regarding industry payments were derived from the Open Payments website (2013-14) which collects information reported by manufacturers and group purchasing organizations (https://www.cms.gov/OpenPayments/ index.html) Surgeons performing <20 fusions from 2011-2012, those without complication data, and those without payment data were excluded from this study. Pearson correlation coefficients and multivariate regression analysis were used to determine the relationship between industry payments, rate of lumbar fusion recommendation, and complication rate.

Results: A total of 1947 spine surgeons (54\% orthopedic, $46 \%$ neurosurgeon) practicing in the United States met our inclusion criteria. Pearson correlation analysis reveals a negligible positive correlation between industry payments and both lumbar fusion recommendation and complication rate $(r=0.09$ and $r=0.02)$. An r-value $>0.30$ is generally accepted as a threshold for weak correlations. Multivariate analysis, illustrated in figure 1 , demonstrated no significant relationship among those same variables.

Discussion/Conclusion: While spine surgeons receive the highest industry payment amounts among all subspecialties, conflict of interest does not appear to have a significant impact on practice patterns or complication rates. 
Poster 26. Can S2-Alar-Iliac (S2AI) Screws Be Placed Accurately Without Fluoroscopy?

Daniel Kang, $M D^{I}$, Jacob Buchowski, $M D^{2}$, Lawrence Lenke, $M D^{3}$, Ronald Lehman, $M D^{4}$, Panya Luksanapruska, $M D^{5}$, Jamal McClendon, Jr., $M D^{2}$, Todd Chapman, $M D^{2}$, Lionel Metz, $M D^{2}$

${ }^{1}$ Madigan Army Medical Center, Tacoma, WA, ${ }^{2}$ Washington University School of Medicine, St. Louis, MO, ${ }^{3}$, New York, NY, ${ }^{4}$, New York, NY, ${ }^{5}$ Department of Orthopedic Surgery, Faculty of Medicine Siriraj Hospital, Bangkoknoi, Bangkok

Background/Introduction: S2AI screw insertion is most often performed with fluoroscopic guidance, however, the freehand S2AI screw insertion provides an alternative technique void of any flouroscopic radiation. However, the accuracy and safety of the freehand S2AI screw technique has not been completely evaluated.

Materials/Methods: Retrospective analysis of adult spinal deformity patients treated at one institution, single surgeon over 4 years (2010-14) with freehand S2AI screw placement, consecutively assessed with intraoperative computed tomography (CT) scan. Each CT image sequence was assessed by 3 spine surgeons, and any pedicle awl or screw violation of the cortical wall was confirmed/graded through consensus agreement. S2AI screw position was graded as acceptable (complete screw within the pelvic/iliac corridor), minor violation $(<=6 \mathrm{~mm})$ and major/ critical violation $(>6 \mathrm{~mm})$. Screws not adequately visualized on the $\mathrm{CT}$ images were excluded.

Results: Overall, there were 190 freehand S2AI screws inserted in 98 patients ( $n=93$ Left; $n=97$ Right) and adequately visualized on intraoperative CT images. There were 5 patients with Right S2AI + Left Iliac ( $n=3$ Left lateral pedicle awl violations), 1 patient Left S2AI + Right iliac screw, and 5 patients with multiple (>2) ipsilateral pelvic fixation points. Overall there was a $9.5 \%$ violation rate ( $\mathrm{n}=8 \mathrm{Left} ; \mathrm{n}=10$ Right). The rate of minor violation was $5.8 \%$ (lateral $n=9,4.7 \%$, average $3.1 \mathrm{~mm}$; medial $n=2,1.1 \%$, average $2.5 \mathrm{~mm}$ ), and major/critical violation was $3.2 \%$ (lateral $\mathrm{n}=3,1.6 \%$, average $10.8 \mathrm{~mm}$; medial $\mathrm{n}=4,2.1 \%$, average $15.6 \mathrm{~mm}$ ). There were 12 pedicle awl violations, all were lateral $(n=8$ Left; $\mathrm{n}=4$ Right). No patients had neurologic, vascular, or visceral complications related to S2AI screw placement.

Discussion/Conclusion: Our study found a 90.5\% accuracy rate for acceptable screw position using freehand S2AI screw insertion without fluoroscopy, with $5.8 \%$ minor and $3.2 \%$ major/ critical cortical wall violations. There were no S2AI screw related complications, and most screws violations were minor and occurred laterally. We found freehand S2AI screw insertion is a safe and reliable method, and obviates the use of fluoroscopic guidance.

Poster 27. National Trends for Primary and Revision Posterior Lumbar Fusions throughout the United States

Comron Saifi, $M D^{1}$, Alex Ha, $M D^{2}$, Alejandro Cazzulino, $B A^{l}$, Ronald Lehman, $M D^{3}$, Lawrence Lenke, $M D^{I}$

\section{${ }^{1}$, New York, NY, ${ }^{2}$, New York , NY, ${ }^{3}$, New York, NY}

Background/Introduction: Given the increasing focus on health care utilization and value-based care, it is essential that the number of primary and revision posterior lumbar fusions per year throughout the United States are determined. Additionally patient demographics and economic data associated with primary and revision lumbar fusions is critical to understanding and improving health care utilization.

Materials/Methods: The data utilized in this study was collected between 2011-2013 across 44 states from the National Inpatient Sample (NIS) database on patients who underwent either primary posterior lumbar fusions (ICD-9-CM 81.07) or revision posterior lumbar fusions (ICD-9-CM 81.37). Demographic and economic data were obtained which included the annual number of surgeries, age, sex, total charges, total costs, length of stay (LOS), insurance type, location, and frequency of routine discharge. The NIS database represents a $20 \%$ sample of discharges from U.S. hospitals, excluding rehabilitation and long-term acute care hospitals, which is weighted to provide national estimates.

Results: The total number of posterior lumbar fusion has progressively decreased $19 \%$ from 127,916 in 2011 to 103,215 in 2013. The mean total cost, including hospital and physician related costs, of lumbar fusion decreased $12 \%$ from $\$ 28,333$ in 2011 to $\$ 24,916$ in 2013 . The mean cost was $\$ 27,170$ per case over the three year study period. The mean LOS remained remained stable at 3.9 days. The mean routine discharge of $62 \%$ also did not vary significantly based on year. The number of revision procedures similarly declined $19 \%$ from 10,717 in 2011 to 8,610 in 2013. Hence the mean national revision burden was $8 \%$. The mean cost of revision posterior lumbar fusion was $\$ 28,937$ over the study period. The mean LOS for patients undergoing revision posterior lumbar fusion was 4.0 days with routine discharge in $64 \%$ of those patients.

Discussion/Conclusion: Throughout the United States there was a progressive decline of both primary and revision posterior lumbar fusions by $19 \%$ over the study period as well as a decrease in total costs for primary posterior fusion surgeries by $12 \%$ between 2012 and 2013. The United States revision burden for posterior lumbar fusion increased from $7 \%$ in 2011 to $9 \%$ in 2013.

Poster 28. Demographics, Mechanisms of Injury, and Concurrent Injuries Associated with Lumbar Spine Injuries: A study of 82,223 patients in the National Trauma Data Bank

Nathaniel Ondeck, B.S. ${ }^{1}$, Nidharshan Anandasivam, $B S^{2}$, Andre Samuel, BBA ${ }^{2}$, Daniel Bohl, $M D, M P H^{3}$, Jonathan Grauer, $M D^{l}$

${ }^{1}$, New Haven, CT, ${ }^{2}$ Yale School of Medicine, New Haven, CT, ${ }^{3}$ Rush University Medical Center, New Haven, CT

Background/Introduction: The present study utilized the National Trauma Data Bank (NTDB) to assess demographics, mechanisms of injury, and concurrent injuries associated with lumbar spine injuries. In addition, it analyzed factors associated with mortality.

Materials/Methods: Adult patients with lumbar spine injury in the 2011 and 2012 NTDB were identified and analyzed; 82,223 patients were included.

Results: The age distribution for lumbar injuries was bimodal, with peaks occurring at 21 and 50 years of age. As expected, the most common mechanism of injury for younger patients was motor vehicle accidents (68\% of patients aged 18-39), and the most common mechanism of injury for older patients was falls (61\% of patients aged 65 and older). Injury Severity Score (ISS) did not vary across the age spectrum. In total, $75 \%$ of patients had at least one concurrent injury (Figure 1). Of orthopaedic injuries, the most common concurrent injuries were thoracic spine fracture $(22 \%)$, cervical spine fracture $(21 \%)$, pelvis fracture $(20 \%)$, and sacral fracture (11\%). Of non-orthopaedic injuries, the most common concurrent injuries were a lung injury (26\%), skull fracture $(20 \%)$ and then intracranial injury $(19 \%)$. A multivariate regression demonstrated that increased injury severity (ISS) was a much stronger predictor of mortality (odds ratio of 64 for patients with ISS greater than 30) than increased age, comorbidity burden, and mechanism of injury.

Discussion/Conclusion: Patients with lumbar spine injuries have high rates of concurrent injuries to the cervical, thoracic and sacral spine as well as the pelvis, lung, and head. Although there is a desire to be judicious with the use of imaging for 
the trauma patient, the high rate of concurrent injuries and the strong association of ISS with death, highlight the importance of identifying and managing such injuries.

Poster 29. Sacral Bone Mineral Density using Opportunistic CT Scans

Ryan Hoel, $M D^{l}$, Charles Ledonio, MD', Takashi Takahashi, $M D^{2}$, David Polly, Jr, $M D^{l}$

${ }^{1}$ University of Minnesota, Minneapolis, MN,${ }^{2}$, Minneapolis, MN

Background/Introduction: Sacral bone mineral density may play a role in the rate of successful sacroiliac and lumbosacral fixation and fusion. There are currently no easy methods for evaluation of sacral bone mineral density (BMD). Lumbar vertebral CT-attenuation in opportunistic CT scans has previously been shown to relate to BMD as assessed by DXA. The goal of this study was to establish an accessible method for sacral BMD analysis using opportunistic CT scans.

Materials/Methods: This study was approved through our institutional review board. A series of 223 consecutive renalprotocol CT scans performed for any reason were retrospectively analyzed using basic image viewing software. Renal CT scans were selected because they include the spine from L1 through the sacrum, and are high resolution images with fine cuts. Sacral CT-attenuations at multiple regions of interest (ROI) were compared to the L1 vertebral CT-attenuation, an internal reference standard previously shown to relate to bone mineral density. The sacral ROI were analyzed to determine regions of higher and lower attenuation. Repeatability was measured by blinded reevaluation of $10 \%$ of the collected data by the same reviewer.

Results: Of the 223 scans evaluated, 112 had sufficient imaging and met inclusion criteria for analysis. All sacral ROI had strong correlations with L1 vertebrae attenuation values $(\mathrm{r}=0.77-0.85 ; \mathrm{p}<.001)$, and most values became stronger when transitional vertebrae were excluded. Sacral attenuation values varied predictably by location and decreased with age. Repeatability of sacral ROI measurements was found to be very good, with standard deviations of $2-7 \%$.

Discussion/Conclusion: This study establishes a method for evaluation of sacral CT-attenuation. Our attenuation values matched topographic variations in sacral BMD found in prior studies. Additionally, as previous studies have shown correlations between lumbar CT-attenuation and bone mineral density as assessed by DXA scan results, and we found strong correlations between lumbar and sacral CT-attenuations, we infer that sacral bone mineral density can be evaluated using this methodology. Furthermore, this method uses opportunistic CT scans, allowing for evaluation of sacral BMD in patients with prior CT scans with no additional radiation exposure.

Poster 30. Rates of Facet Joint Fusion and Posteromedial Fusion Following Elective Single-Level Posterior Instrumented Lumbar Spinal Fusion Surgery

David Kim, $M D^{l}$, Riya Joshi, MPH, MBBS ${ }^{2}$, Kevin Baker, Ph.D ${ }^{3}$, Gyu Ho Lee, $B A^{4}$, Daniel Park, $M D^{5}$, Paul Arnold, $M D^{6}$, Rick Sasso, $M D^{7}$, Jeffrey Fischgrund, $M D^{3}$

${ }^{1}$ New England Baptist Hospital, Boston, MA, 2, Waltham, MA, ${ }^{3}$, Royal Oak, Michigan, ${ }^{4}$, Carlisle, PA, ${ }^{5}$, Royal Oak, MI, ${ }^{6}$, Kansas City, KS, ${ }^{7}$, Carmel, IN

Background/Introduction: Following lumbar fusion surgery, radiologic evidence of successful fusion has traditionally been based on bridging bone spanning the intertransverse process space (posterolateral fusion; PLF) or adjacent vertebral body endplates (interbody fusion; IBF). In many cases of unsuccessful PLF/IBF, postoperative computed tomography $(\mathrm{CT})$ demonstrates bridging bone across the facet joints or connecting the transverse process to the caudal superior articular facet. These facet joint fusions (FJF) or so-called posteromedial fusions (PMF) have been proposed as stable outcomes despite limited biomechanical and clinical data. The purpose of this study was to determine prospective CT-based comparative FJF, PMF, PLF, and IBF fusion rates and outcomes following single-level posterior lumbar instrumented fusion.

Materials/Methods: 241 patients underwent single-level posterior lumbar instrumented fusion with local autograft bone using either the PLF or PLF with interbody fusion (PLF+IBF) technique as part of a prospective, multi-center, randomized controlled trial. Fusion was assessed at 6-month and 12-month postoperative time points by two independent reviewers. Clinical outcome measures included visual analogue scale (VAS) scores for back pain and leg pain, Oswestry Disability Index (ODI), and SF-36.

Results: 231 patients and 191 patients completed 6-month and 12-month follow-up respectively. At 12 months, 35.7\% of PLF patients demonstrated bilateral PLF fusion, and $50.3 \%$ demonstrated at least unilateral fusion. Among PLF+IBF patients, $5.4 \%$ demonstrated successful IBF and bilateral PLF fusion, and $50.8 \%$ demonstrated fusion of at least one region (IB or at least one PLF side). At 6 months, $8.4 \%$ of patients with intact bilateral facet joints demonstrated bilateral FJF and/or PMF, and $31.3 \%$ demonstrated at least unilateral FJF/PMF. At 12 months, $52.7 \%$ demonstrated successful bilateral FJF/PMF, and $69.1 \%$ demonstrated at least unilateral FJF/PMF. All fusion groups (including patients with only FJF) demonstrated equivalent improvement in back and leg pain scores, ODI and SF-36. No differences were observed comparing patients with successful PLF/IBF versus FJF only. Similarly, there was no difference between PLF/IBF and FJF fusion groups in rates of implant loosening, complications, or reoperation.

Discussion/Conclusion: In terms of short-term clinical outcome and avoidance of pedicle screw loosening or implant-related complications, successful facet joint fusion (FJF) provides success rates comparable to posterolateral or interbody fusion.

Poster 31. Spinous Process Fractures after Placement of an Interspinous Process Spacer Device: Insight into the Mode of Action of an Interspinous Device

William Lavelle, $M D^{l}$, Dongliang Wang, $P h D^{l}$, Ian Madom, $M D^{1}$; Henaku Yirenkyi, $M D^{2}$, Fred Geisler, $M D, P h D^{3}$

${ }^{1}$, Syracuse, NY, ${ }^{2}$ SUNY Upstate Medical University, Syracuse, NY, ${ }^{3}$ Rhausler, Inc., San Carlos, CA

Background/Introduction: Interspinous devices may be less invasive to manage mild to moderate lumbar stenosis. Reports of spinous process fractures found rate to be $22 \%$ and rates of $52 \%$ with spondylolysithesis questioning impact on patient outcomes. Proposed device mechanism forces spinous processes apart and causes distraction; appears patients would have poorer outcomes because fracture reduces implant's ability to flex vertebra forward. Our study determined the effect of spinous process fractures on functional outcomes.

Materials/Methods: In a prospective multicenter IDE study with patients aged $45<$ with moderate lumbar spinal stenosis, compared Superion device to X-stop device in non-inferiority study. 344 subjects randomized, enrolled and operative data collected. At follow-up visits, subjects completed questionnaires 
evaluating disability, function, pain, quality of life and satisfaction. Patients underwent standing anterior/posterior, lateral and flexion/ extension lateral lumbar spine views reviewed by independent radiologist. Stepwise multivariate regression analysis completed looking at factors affecting patient ODI at 1 year. Identified factors sub-examined in patients with and without postoperative spinous process fractures.

Results: 263 subjects were without fracture and 81 with fracture. Average ODI at 1 year for subjects with fracture 1.465 units more than patients without fracture; not statistically significant $(p=0.403)$. Subjects without fracture, ODI at 1 year significantly affected by foraminal height difference between week 6 and preoperatively $(\mathrm{p}=0.031)$, but not flexion/extension (FE) motion week $6(\mathrm{p}=0.800)$. Every $1 \mathrm{~mm}$ increase of foraminal height, ODI at 1 year decreased 3.455 units. Subjects with fractures, ODI at 1 year more significantly affected by FE rotation week $6(p=0.007)$, but not by foraminal height increase between week 6 and pre-operatively $(\mathrm{p}=0.766)$. For every unit increase of FE rotation at week 6 , ODI at 1 year increased 2.725 units. FE motion noted on pre-op radiograph did not predict occurrence of spinous process fractures.

Discussion/Conclusion: Spinous process fractures after placement of interspinous devices are under recognized. We found more robust outcomes for patients with fractures. Examining factors associated with better outcome scores, arresting spinal motion may represent plausible mode of action for implants as opposed to foraminal distraction. Patients with painful spinous fractures may limit lumbar range of motion; thereby, limiting flexion and extension.

Poster 32. Differences in Clinical Outcomes between Isthmic and Degenerative Spondylolisthesis following a Minimally Invasive Transforaminal Lumbar Interbody Fusion

Dustin Massel, $B S^{l}$; Benjamin Mayo, $B A^{l}$, William Long, $B A^{l}$, Krishna Modi, $B S^{l}$, Bryce Basques, $M D^{l}$, Daniel Bohl, $M D, M P H^{2}$, Kern Singh, $M D^{l}$

${ }^{1}$ Rush University Medical Center, Chicago, IL, ${ }^{2}$ Rush University Medical Center, New Haven, CT

Background/Introduction: MIS TLIF is a common surgical procedure for treatment of degenerative spondylolisthesis (DS). However, MIS TLIF remains controversial for the treatment of isthmic spondylolisthesis (IS). Few studies have compared the surgical outcomes of IS and DS following MIS TLIF. The purpose is to compare clinical outcomes of patients with IS or DS following a MIS TLIF.

Materials/Methods: A prospectively maintained surgical database of patients who underwent a primary 1-level MIS TLIF for spondylolisthesis between 2007-2015 was reviewed. Patients with IS underwent a MIS TLIF with bilateral tubular approach and bilateral interbody placement versus the DS cohort underwent unilateral approach and single interbody cage placement. Patient demographics and preoperative characteristics were assessed using independent sample t-tests and Chi-square analysis for continuous and categorical variables, respectively. Postoperative outcomes were analyzed using Poisson regression with robust error variance (binary outcomes) or linear regression (continuous outcomes) adjusted for patient demographics, preoperative characteristics, and perioperative variables.

Results: A total of 245 patients were identified (IS: $n=65$ (26.5\%); DS: $n=180(73.5 \%))$. IS patients were younger $(47.6$ vs 56.6 years, $\mathrm{p}<0.001)$, had a lower comorbidity burden ( 2.2 vs $3.6, \mathrm{p}<0.001)$, and had a greater incidence of L5-S1 operations $(69.2 \%$ vs $16.7 \%, \mathrm{p}<0.001)$ than the DS cohort. Patients with IS experienced longer operative times (147.8 vs $125.6 \mathrm{~min}, \mathrm{p}=0.003$ ), greater estimated blood loss (EBL; 105.6 vs $72.5 \mathrm{~mL}, \mathrm{p}<0.001$ ), and lower, but not statistically significant, arthrodesis rates compared to the DS cohort (Table 2).

Discussion/Conclusion: Despite IS patients being younger and having a lower comorbidity index than the DS cohort, similar clinical outcomes were observed following MIS TLIF. The increased estimated blood loss in IS procedures may be due to the increased complexity of the surgical technique compared to that required for DS, but was still not clinically meaningful. The results of this study suggest MIS TLIF is an appropriate treatment option for IS patients despite the increased instability inherent with IS. Further studies analyzing preoperative risk factors are needed to better elucidate which IS patients are the most appropriate candidates for MIS TLIF to ensure adequate arthrodesis rates.

\section{Poster 33 - Retracted}

Poster 34. Front Occupant Thoraco-lumbar Spine Injuries Resulting from Frontal Impact Motor Vehicle Collisions, Correlated to AO Spine Classification

\author{
Hesham Soliman, $M D^{l}$, Narayan Yoganandan, $P h D^{2}$, Shekar \\ Kurpad, $M D, P h D^{3}$
}

${ }^{1}$ Medical College of Wisconsin, Wauwatosa, Wisconsin, ${ }^{2}$ Medical College of Wisconsin, Milwaukee, Wisconsin, ${ }^{3} \mathrm{MCW}$, Milwaukee, Wisconsin

Background/Introduction: The relatively recently modified AO Spine classification for thoraco-lumbar spine fractures was put forward as a means for predicting the need for surgical intervention. The purpose of this study is to further validate the AO Spine classification as a predictor of the severity of injury.

Materials/Methods: Retrospective review of prospective collected data in the CIREN database that includes occupants of motor vehicles that have sustained moderate to severe injuries from crashes.

Results: Out of a total of 4,572 occupants in the CIREN database, narrowed down to 2112 that sustained injury in newer vehicles (2000 models and newer). 631 were identified as having T or L Spine injury. Only 299 had major injuries. We have isolated 102 major $\mathrm{T}$ and $\mathrm{L}$ spine fractures due to high ranking impacts to the front of the vehicle. According to AO Classification, fractures that are likely unstable warranting surgical intervention demonstrated a trend to increase with age, to peak at $60-69 \mathrm{yr}$, while A1,A2 (69 cases,67.6\%) did not. All regions demonstrated a more frequent $\mathrm{A} 1, \mathrm{~A} 2$, followed by $\mathrm{A} 3, \mathrm{~A} 4$, followed by $\mathrm{B}$ then $\mathrm{C}$ - with the exception of $\mathrm{T}$ Spine injuries that demonstrated more extension injuries more than flexion and distraction. According to Barrier equivalent speed (BES), the moderate and high speed groups were comparable in terms of A class frequency of injury. The moderate speed group had double the incidence of B $(50 \%)$ and $C(66.6 \%)$ injuries, compared to high speed group, while the low speed group interestingly showed a $14 \%$ incidence of total A injuries, and $25 \%$ of the total B injuries.

Discussion/Conclusion: We confirm the increased frequency of extension injuries in the $\mathrm{T}$ Spine, while flexion distraction injuries were more common in TLJ. An interesting finding was that severe injuries per $\mathrm{AO}$ Classification did not correlate with the speed of frontal impact, potentiating the expected role of rotational forces at the moment of impact. $25 \%$ of B Class injuries occurred in the low speed group per BES. This warrants further biomechanical testing and analysis. 
Poster 35. Multimodal versus Patient Controlled Analgesia Following Transforaminal Lumbar Interbody Fusion

Daniel Bohl, MD, MPH $H^{1}$, Junyoung Ahn, BS $S^{2}$, Philip Louie,

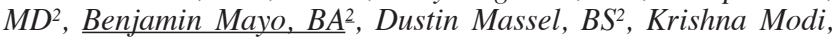
$B S^{2}$, William Long, $B A^{2}$, Kern Singh, $M D^{2}$

${ }^{1}$ Rush University Medical Center, New Haven, CT, ${ }^{2}$ Rush University Medical Center, Chicago, IL

Background/Introduction: There has been increasing use of multimodal analgesia (MMA) following spinal procedures. The purpose of this study is to compare postoperative narcotic consumption between patients receiving MMA and patients receiving another common postoperative pain control mechanism, patient-controlled analgesia (PCA), following a minimally invasive (MIS) transforaminal lumbar interbody fusion (TLIF).

Materials/Methods: A prospectively maintained database patients undergoing MIS TLIF for degenerative spondylolisthesis was reviewed. Patients were categorized as having received either MMA (more recently treated patients) or PCA (historic controls). The MMA protocol is described in Table 1. Total inpatient narcotic consumption in oral morphine equivalent (OME) was calculated for each patient. This value was divided by the hospital length of stay to calculate the average rate of narcotic consumption over the inpatient stay (in OME/hour). Narcotic dependence at the first and second postoperative visits was determined based on filling of narcotic prescriptions during the weeks following each of these visits. The rates of inpatient narcotic consumption and narcotic dependence at the first and second postoperative visits were compared between patients who received MMA and patients who received PCA.

Results: A total of 139 patients met inclusion criteria. Of these, $39(28.1 \%)$ received MMA and 100 (71.9\%) received PCA. Baseline characteristics were not statistically different between groups (Table 2). Patients who received MMA had a lower rate of inpatient narcotic consumption (2.8 versus $5.3 \mathrm{OME} / \mathrm{hour}$, $\mathrm{p}<0.001)$ and less likely to have nausea/vomiting during the inpatient stay than patients who received PCA $(20.5 \%$ versus $48.0 \%, \mathrm{p}=0.003)$. There was no difference in the rate of narcotic dependence at the first $(50.0 \%$ versus $33.7 \%, \mathrm{p}=0.079)$ and second $(66.7 \%$ versus $51.0 \%, \mathrm{p}=0.095)$ postoperative visits between patients who received MMA and those who received PCA.

Discussion/Conclusion: The results of the present study suggests that patients undergoing MIS TLIF may have lower narcotic consumption during the inpatient stay with use of MMA than with use of PCA, resulting in a decrease in the number of episodes of nausea/vomiting during the inpatient stay. However, this difference does not appear to result in a difference in the risk for narcotic dependence during the months following surgery.

\section{Poster 36. Upright MRI Lumbar Spine}

Ha Nguyen, MD, Ninh Doan, MD, PhD, Saman Shabani, MD, Jamie Baisden, MD, Christopher Wolfla, MD, Barry Shender, PhD, Brian Stemper, PhD

\section{Medical College of Wisconsin, Milwaukee, Wisconsin}

Background/Introduction: Lumbar back pain and radiculopathy are common diagnoses. Unfortunately, conventional MRI findings and clinical symptoms do not necessarily correlate in the lumbar spine. With upright imaging, disc pathologies or foraminal stenosis may become more salient, leading to improvements in diagnosis.

Materials/Methods: Seventeen adults (10 asymptomatic and 7 symptomatic volunteers) provided their informed consent and participated in the study. A 0.6 T upright MRI scanned each adult in the seated position. Parameters were obtained from the $\mathrm{L} 2 / 3$ level to L5/S1 level, including those pertaining to the foramen (cross sectional area (CSA), height, mid-disc width, width, thickness of ligamentum flavum), disc (bulge, height, width), vertebral body (height and width) and alignment (lordosis angle, wedge angle, lumbosacral angle). Each parameter was compared based on spinal level and volunteer group using twofactor ANOVA. Bonferroni post-hoc analysis was used to assess differences between individual spinal levels.

Results: Mid-disc width accounted for $56 \%$ of maximum foramen width in symptomatic volunteers and over $63 \%$ in asymptomatic volunteers. Disc bulge was $48 \%$ greater in symptomatic volunteers compared to asymptomatic volunteers. Foraminal CSA was generally smaller in symptomatic volunteers compared to asymptomatic volunteers, particularly at the L4-L5 and L5-S1 spinal levels. Ligamentum flavum thickness generally increased from cranial to caudal spinal levels, where the L4-L5 and L5-S1 spinal levels were significantly thicker than the L1-L2 spinal level.

Discussion/Conclusion: The data establishes normal parameters for upright MRI anatomy, and implies that upright MRI could be a useful diagnostic option, as it can delineate pertinent differences between symptomatic volunteers and asymptomatic volunteers, especially with respect to foraminal geometry.

\section{Poster 37. RLDH: Repeat Discectomy Versus Fusion}

Jian Guan, MD, Vijay Ravindra, MD, MSPH, Meic Schmidt, MD, MBA; Andrew Dailey, MD, Robert Hood, MD, Erica Bisson, $M D, M P H$

\section{University of Utah, Salt Lake City, UT}

Background/Introduction: RLDH is a significant cause of morbidity in patients undergoing lumbar discectomy, occurring in up to $18 \%$ of cases. While repeat discectomy is often successful in treating these patients, concern over repeat RLDH may lead surgeons to advocate instrumented fusion even in the absence of instability.

Materials/Methods: We used the National Neurosurgery Quality and Outcomes Database (N2QOD) to assess outcomes of patients who underwent repeat discectomy versus instrumented fusion at a single institution from 2012 to 2015. Primary outcomes included Oswestry Disability Index (ODI) score, Visual Analog Scale (VAS) score, and quality-adjusted life year (QALY) measures. Secondary outcomes included hospital length of stay, discharge status, and hospital charges.

Results: We identified 25 repeat discectomy and 12 instrumented fusion patients with 3- and 12-month follow-up records. The groups had similar improvements in ODI, VAS, and QALY measures at 3 and 12 months. Patients in the instrumented fusion group had significantly longer hospitalizations (3.7 days vs. 1.0 days, $\mathrm{p}<0.001)$ and operative times $(229.6$ minutes vs. 82.7 minutes, $\mathrm{p}<0.001)$. They were also more likely to be female $(\mathrm{p}=0.020)$ and to discharge to inpatient rehabilitation instead of home $(\mathrm{p}=0.036)$. Hospital charges for the instrumented fusion group were also significantly higher (\$54,458.29 vs. $\$ 11,567.05, \mathrm{p}<0.001)$. Rates of re-operation were higher in the repeat discectomy group ( $12 \%$ vs. $0 \%$ ), but the difference was not statistically significant $(\mathrm{p}=0.211)$.

Discussion/Conclusion: Both approaches offer improvements in clinical outcomes at short-term follow-up. While repeat discectomy offers several short-term advantages, instrumented fusion may reduce rates of re-operation. Procedure selection should be individualized for each patient's needs to maximize benefit and minimize risk. 
Poster 38. Syngeneic Iliac Crest and Femoral Allografts Compared to Iliac Crest Autograft in a Rat Spinal Fusion Model

Benjamin Elder, MD, $P h D^{1}$; Christina Holmes, $P h D^{2}$, Wataru Ishida, $M D^{1}$, Timothy Witham, $M D^{2}$

${ }^{1}$ Johns Hopkins University School of Medicine, BALTIMORE, Maryland, ${ }^{2}$ Johns Hopkins University School of Medicine, Baltimore, MD

Background/Introduction: Even within animal models, autograft iliac crest bone remains the "gold standard" in lumbar spinal fusion procedures. However, the use of inbred rodent strains allows for the use of syngeneic allograft bone from a wider variety of donor sites. While femoral head allografts have been successfully used in a number of clinical spinal fusion studies (S. Gibson, 2002, Spine (Phila. Pa. 1976). 27: 1599; J. Urrutia, 2013, Orthop. Traumatol. Surg. Res. 99: 413; D.A. Brown, 2014, Spine (Phila. Pa. 1976). 39:E902), little data is available comparing the performance of femoral grafts to other types of autograft or allograft bone. In this study, we compared the use of autogenous iliac crest with syngeneic femoral and iliac crest allograft bone in the rat model of lumbar spinal fusion.

Materials/Methods: Bilateral posterolateral intertransverse process lumbar spinal fusion surgery was performed at a single level (L5-L6) on 45 female Lewis rats divided into three experimental groups: iliac crest autograft $(n=15)$; syngeneic iliac crest allograft $(n=15)$; and syngeneic femoral allograft bone $(n=15)$, all consisting of corticocancellous bone. Fusion was evaluated via microCT analysis, manual palpation and histology at 8 weeks post-operation.

Results: Femoral syngeneic allografts were the largest at 0.42 $\pm 0.10 \mathrm{~g}$, compared to iliac crest allografts at $0.35 \pm 0.13 \mathrm{~g}$ and iliac crest autografts at $0.15 \pm 0.10 \mathrm{~g}$. However, allograft iliac crest bone displayed the highest fusion rates $(37.5 \%$ bilaterally fused, $37.5 \%$ unilaterally fused and $25 \%$ non-fused) via manual palpation, compared to the autograft group ( $15 \%$ bilaterally fused, $54 \%$ unilaterally fused, $31 \%$ non-fused) and the femoral allograft group (7\% bilaterally, $40 \%$ unilaterally, $53 \%$ non-fused).

Discussion/Conclusion: While the femoral syngeneic allografts were the largest initially, this did not yield larger fusion mass volumes compared to those produced by the smaller iliac crest grafts. Interestingly, femoral allografts showed lower rates of solid fusion, via manual palpation, than both iliac crest autograft and allograft groups. As in previous studies, CT fusion scores did not directly correlate with manual palpation fusion scores.

\section{Poster 39. Spine Thirty-Day Readmission Rates}

\section{James Bernatz, BS $^{1}$, Paul Anderson, $M D^{2}$}

${ }^{1}$ University of Wisconsin School of Medicine \& Public Health, Madison, Wisconsin, ${ }^{2}$ University of Wisconsin, Madison, WI

Background/Introduction: The rate of 30-day readmissions is rapidly gaining significance as a quality metric and is being increasingly used to evaluate performance. An analysis of the present 30-day readmission rate in spine literature is needed to aid development of policies to decrease the frequency of readmissions. The authors examine two questions: 1) What is the 30-day readmission rate as reported in spine literature? 2) What study factors impact the rate of 30-day readmissions?

Materials/Methods: Four electronic databases were searched for articles. A systematic review and meta-analysis was performed to assess the current 30-day readmission rate in spine surgery. Thirteen studies met inclusion criteria. The readmission rate as well as data source, time from enrollment, sample size, demographics, procedure type and spine level, risk factors for readmission, and causes of readmission were extrapolated from each study.

Results: The pooled 30-day readmission rate was 5.5\% (95\% CI $4.2 \%-7.4 \%)$. Studies from single institutions reported the highest 30-day readmission rate at 6.6\% (95\% CI 3.8\%-11.1\%), while multicenter studies reported the lowest at $4.7 \%$ (95\% CI $2.3 \%-9.7 \%$ ). Time from enrollment had no statistically significant effect on the 30-day readmission rate. Studies including all spinal levels had a higher 30 -day readmission rate $(6.1 \%, 95 \%$ CI $4.1 \%-8.9 \%)$ than exclusively lumbar studies $(4.6 \%, 95 \%$ CI 2.5\%-8.2\%); however, the difference between the 2 rates was not statistically significant $(\mathrm{p}=0.43)$. The most frequently reported risk factors associated with an increased odds of 30-day readmission on multivariate analysis were an American Society of Anesthesiology score of 4+, operative duration, and Medicare/ Medicaid insurance. The most common cause of readmission was wound complication (39.3\%).

Discussion/Conclusion: The 30-day readmission rate following spinal surgery is between 4.2 and 7.4 percent. The range, rather than the exact result, should be considered given the significant heterogeneity among studies, which indicates that there are factors such as demographics, procedure types, and individual institutional factors that are important and that affect this outcome variable. The pooled analysis of risk factors and causes of readmission is limited by the lack of reporting in most of the spine literature.

Poster 40. Pre-operative Narcotic Utilization: Accuracy of Self-Reporting Prior to Spinal Surgery

Junyoung Ahn, $B S^{1}$, Daniel Bohl, $M D, M P H^{2}$, Islam Elboghdady, $B A^{l}$, Dustin Massel, $B S^{l}$; Benjamin Mayo, $B A^{l}$, Krishna Modi, $B S^{l}$, William Long, $B A^{1}$, Kern Singh, $M D^{l}$

${ }^{1}$ Rush University Medical Center, Chicago, IL , ${ }^{2}$ Rush University Medical Center, New Haven, CT

Background/Introduction: Little is known regarding the accuracy of reporting preoperative narcotic utilization in spinal surgery. The purpose is to compare postoperative narcotic consumption between preoperative narcotic utilizers who do and do not accurately self-report preoperative utilization.

Materials/Methods: Patients who underwent anterior cervical discectomy and fusion (ACDF), minimally invasive (MIS) lumbar discectomy (LD), or MIS transforaminal lumbar interbody fusion (TLIF) between 2013-2014 were prospectively identified. Accuracy of self-reporting of preoperative narcotic consumption was determined utilizing the Illinois Prescription Monitoring Program (ILPMP). Total inpatient narcotic consumption during postoperative days 0 and 1 were compared according to demographics and preoperative narcotic reporting accuracy. Similarly, the proportion of patients who continued to be dependent on narcotic medications at each postoperative visit was compared according to demographics and preoperative narcotic reporting accuracy.

Results: A total of 195 patients met inclusion criteria. Of these, $25 \%$ did not utilize narcotics preoperatively, while $47 \%$ and $28 \%$ did do so with accurate and inaccurate reporting, respectively. Patients who utilized narcotics preoperatively were more likely to demonstrate elevated inpatient narcotic consumption (adjusted $\mathrm{RR}=5.3$, 95\% CI 1.4-20.1, $\mathrm{p}=0.013$ ); however, were no more or less likely to be dependent on narcotic medications at the first $(\mathrm{p}=0.618)$ or second $(\mathrm{p}=0.798)$ postoperative visits. The preoperative factors independently associated with elevated inpatient narcotic consumption were Worker's compensation status and procedure type. The preoperative factors independently associated with narcotic dependence at the first postoperative visit were female sex, Worker's compensation status, and procedure 
type. The only preoperative factor that was independently associated with narcotic dependence at the second postoperative visit was procedure type.

Discussion/Conclusion: Our findings suggest that determining the actual preoperative narcotic utilization in patients who undergo spine surgery may help optimize postoperative pain management. Approximately $75 \%$ of patients utilized narcotics preoperatively and demonstrated significantly higher inpatient narcotic consumption. Finally, the postoperative narcotic consumption was independent of the self-reporting accuracy of preoperative narcotic utilization. These findings suggest that corroboration of patients' self-report of preoperative narcotic utilization with other sources of information (family members, narcotic registries, etc.) may help to minimize narcotic requirements thereby potentially improving the management of postoperative pain.
Disclaimer: The Journal of Neurosurgery Publishing Group (JNSPG) acknowledges that the preceding abstracts and poster sessions are published verbatim as submitted and did not go through either the JNSPG's peer-review or editing process. 